\title{
Estudos numéricos para o problema da tomografia por impedância elétrica
}

\author{
Juan Carlos Zavaleta Aguilar
}

TESE APRESENTADA

AO

INSTITUTO DE MATEMÁTICA E ESTATÍSTICA

DA

UNIVERSIDADE DE SÃO PAULO

PARA

OBTENÇÃO DO TÍTULO

DE

DOUTOR EM CIÊNCIAS

\begin{abstract}
Área de Concentração: Matemática Aplicada
Orientador: Prof. Dr. Nelson Mugayar Kuhl
\end{abstract}

Durante o desenvolvimento deste trabalho o autor recebeu auxilio financeiro do CNPq

São Paulo, 06 de Abril de 2009 


\title{
Estudos numéricos para o problema da tomografia por impedância elétrica
}

\author{
Este exemplar corresponde à redação \\ final da tese devidamente corrigida \\ e defendida por Juan Carlos Zavaleta Aguilar \\ e aprovada pela Comissão Julgadora.
}

Banca Examinadora:

- Nelson Mugayar Kuhl (orientador) - IME - USP.

- Jose Jaime da Cruz - POLI - USP.

- Vanessa Rolnik Artioli - FFCLRP - USP.

- Saulo Rabello Maciel de Barros - IME - USP.

- Raúl Gonzales Lima - POLI - USP. 


\section{Agradecimentos}

Em primeiro lugar agradeço a Deus por permitir a culminação deste trabalho e assim dar mais um passo na minha formação acadêmica.

Agradeço em forma muito especial a minha esposa Fátima, que tem sido uma companheira em todas as horas pela sua paciência e dedicação em todos os momentos que foi preciso.

Agradeço as minhas filhas Milena, Laila e Natalie porque elas constituem a minha grande motivação para alcançar os objetivos traçados.

Agradeço aos meus pais Américo e Rita e aos meus irmãos René e Elí por sempre terem acreditado em mim.

Agradeço ao meu orientador, professor. Dr. Nelson Mugayar Kuhl pelo apoio e paciência na orientação e aos muitos conhecimentos adquiridos com ele que fizeram possível a culminação deste trabalho.

Agradeço aos professores que formaram minha banca, os quais fizeram importantes contribuições para o aperfeiçoamento do trabalho.

Agradeço aos professores do Instituto de Matemáticas e Estatística com os quais realizei as disciplinas.

Agradeço aos meus amigos e colegas (Jorge, Valentin, Anderson, Gerard) pelos gratos momentos compartilhados. Agradeço também ao grupo de Tomografia por Impedância Elétrica da POLI, em especial, a Julio pelos muitos conhecimentos adquiridos.

Agradeço ao pessoal administrativo do IME pela atenção e gentileza.

Agradeço ao CNPq pelo apoio financeiro. 


\section{Resumo}

Este trabalho estuda a técnica de reconstrução de imagens conhecido como tomografia por impedância elétrica em um domínio bidimensional. Esta técnica consiste na alocação de eletrodos na fronteira do volume e uma fonte injeta padrões de corrente através dos eletrodos e medem-se as voltagens resultantes na fronteira. Com estes dados estima-se a condutividade (ou resistividade) do interior do domínio criando-se uma imagem do mesmo. A tomografia por impedância elétrica é um problema inverso e mal posto no sentido de Hadamard. Estudam-se diversos métodos de solução para resolver o problema direto usando métodos numéricos como diferenças finitas e volumes finitos. Proporemos os métodos numéricos a serem aplicados na solução do problema direto os quais serão testados com problemas onde a solução analítica é conhecida. Posteriormente aplicaremos os métodos propostos ao problema especifico. Uma questão importante na reconstrução de imagens é propor a maneira como aproximar o Jacobiano (ou matriz de sensibilidade) do problema, assim desenvolvemos uma técnica para a aproximação do mesmo usando os dados fornecidos pelo problema direto.

Palavras-Chave: Tomografia por impedância elétrica, métodos de regularização, métodos numéricos. 


\begin{abstract}
In this work is studied the technique of reconstruction of images known as electrical impedance tomography for a two-dimensional domain. This technique consists in the allocation of electrodes on the border of the volume and a source injects patterns of current through the electrodes and then measuring voltages through the other electrodes. With these data it is estimated the conductivity (or resistivity) on the interior of the domain and an image is create of it. The electrical impedance tomography is an inverse and ill conditioned problem in the Hadamard sense. In this work, is studying some numerical methods to solve the direct problem and are applied numerical methods such as the finite difference method and the finite volume method. It is proposed some numerical methods to solve the direct problem which will be tested with analytical problems where the solution is known. Later, apply the methods proposed to the specific issue. An important issue in the reconstruction problems is about the Jacobian (or sensitivity matrix) aproximation, thus proposing a technique for the calculation of even using the data provided by the direct problem.
\end{abstract}

Keywords: Electric impedance tomography, regularization methods, numerical methods. 


\section{SUMÁRIO}

LISTA DE ABREVIATURAS vi

LISTAS DE SÍMBOLOS vii

LISTAS DE FIGURAS Xii

LISTAS DE TABELAS Xiv

1. INTRODUÇÃO 1

1.1 Descrição do Problema............................................................................... 1

1.2 Revisão Bibliográfica................................................................................. 3

1.3 O Problema Inverso de Condutividade é mal posto.................................... 5

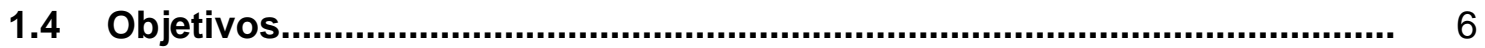

1.5 Divisão da Tese........................................................................................... 7

2. FUNDAMENTOS TEÓRICOS 8

2.1 Equações governantes............................................................................... 8

2.2 O Problema Direto......................................................................................... 9

2.3 A Coleta de dados na TIE......................................................................... 16

2.4 O Problema Inverso......................................................................................... 18

3. ESTUDOS NUMÉRICOS USANDO O EIDORS 27

3.1 Características da malha............................................................................ 27

3.2 Tratamento do Problema Direto................................................................. 29

3.3 Tratamento do Problema Inverso............................................................ 32 
4. ESTUDOS NUMÉRICOS DO PROBLEMA DIRETO

4.1 O Problema de Difusão com Coeficientes Contínuos em Coordenadas Polares 35

4.2 O Problema de Difusão com Coeficientes Descontínuos 45

4.2.1 Discretização por volumes finitos modificados em problemas de dimensão 1

4.2.2 Discretização por volumes finitos modificados em problemas de dimensão 2.

4.2.3 Solução numérica da equação de difusão com coeficientes descontínuos em coordenadas polares. 58

4.3 Aproximação da solução na fronteira 84

5. ESTUDOS NUMÈRICOS NA TIE 87

5.1 A Malha. 87

5.2 O Problema Direto 88

5.3 Comparações das voltagens 90

5.4 O Jacobiano............................................................................................ 93

5.5 Aproximação do Fluxo nos nós............................................................ 96

5.6 O Jacobiano em termos do Fluxo...................................................... 98

5.7 Aproximação Gauss-Newton............................................................... 101

6. CONCLUSÕES

APÊNDICE A - TRATAMENTO DA CONDIÇÃO DE FRONTEIRA TIPO NEUMANN 


\section{LISTA DE ABREVIATURAS}
AA
Método numérico da média aritmética.
Bi-PGC
Método dos Bi-gradientes conjugados com pré - condicionamento.
$d$-bar
Método $d$-barra.
DOT
Tomografia ótica difusa.
EIDORS
Electrical Impedance and Diffuse Optical tomography Reconstruction Software
GC
Gradientes conjugados.
HA
Método que usa a média harmônica.
HIA
Método que usa a média harmônica modificada.
ITM
Indução tomográfica.
LU
Método numérico para resolver um sistema linear
MCE
Modelo completo de eletrodos.
MVFM
Método dos volumes finitos modificados.
PGC
Gradientes conjugados com pré-condicionamento.
SVD
Método numérico da decomposição dos valores singulares.
TC
Tomografia computadorizada.
TIE
Tomografia por impedância elétrica.
TCE
Tomografia por capacitância elétrica.
TOAST
Tomografia em óptica.
UMIST
University of Manchester Institute of Science and Technology. 


\section{LISTA DE SÍMBOLOS}

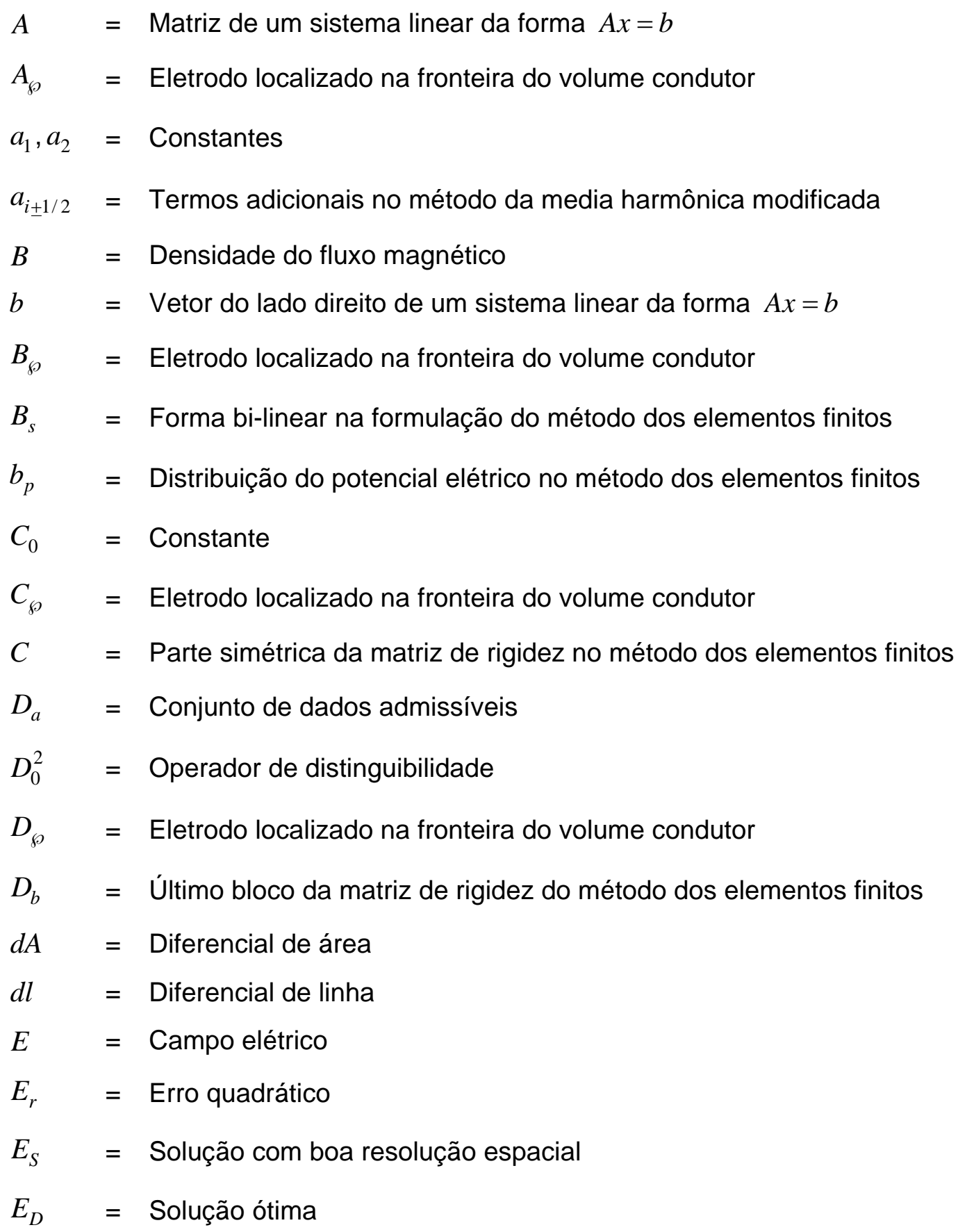


viii

$e_{l} \quad=\quad l$-ésimo eletrodo localizado na fronteira do volume condutor

$e_{i}^{j} \quad=$ Componente de erro no $i$-esimo eletrodo e na $j$-esima iteração

$e_{\max }=$ Erro relativo na norma do máximo

$e_{L^{2}}=$ Erro relativo na norma $L^{2}$

$F=$ Função com inversa contínua

$f=$ Função fonte do problema de difusão

$F_{i j} \quad=$ Aproximação dos fluxos

$g=$ Função que produz os valores na condição de Dirichlet

$H^{p} \quad=$ Espaço de Sobolev

$h=$ Função que produz os valores na condição de Neumann

$h_{x}=$ Tamanho do passo na direção $x$ em coordenadas cartesianas

$h_{y}=$ Tamanho do passo na direção $y$ em coordenadas cartesianas

$h_{r} \quad=\quad$ Tamanho do passo na direção $r$ em coordenadas polares

$h_{\theta}=$ Tamanho do passo na direção $\theta$ em coordenadas polares

$I=$ Padrão de corrente

$I^{d}=d$-esimo padrão de corrente

$I_{\psi} \quad=$ Padrão de corrente que produz o potencial $\psi$

$I_{\phi} \quad=$ Padrão de corrente que produz o potencial $\phi$

$J=$ Jacobiano

$J_{d l, k}=$ Componentes do Jacobiano relacionando o $l$-ésimo eletrodo, o $k$-ésimo padrão de corrente e o $n$-ésimo volume finito.

$j=$ Densidade de corrente

$J^{s} \quad=\quad$ Fonte de corrente

$K_{i j}^{H}=$ Temo correspondente a média harmônica dos coeficientes $\sigma_{i j}$ num volume finito

$L=$ Índice que indica o número de eletrodos alocados na fronteira do volume

$L_{i} \quad=$ Polinômios de Lagrange

$L D=$ Lado direito de um sistema linear em forma matricial

$O\left(h^{p}\right) \quad=$ Termos de ordem $p$ 


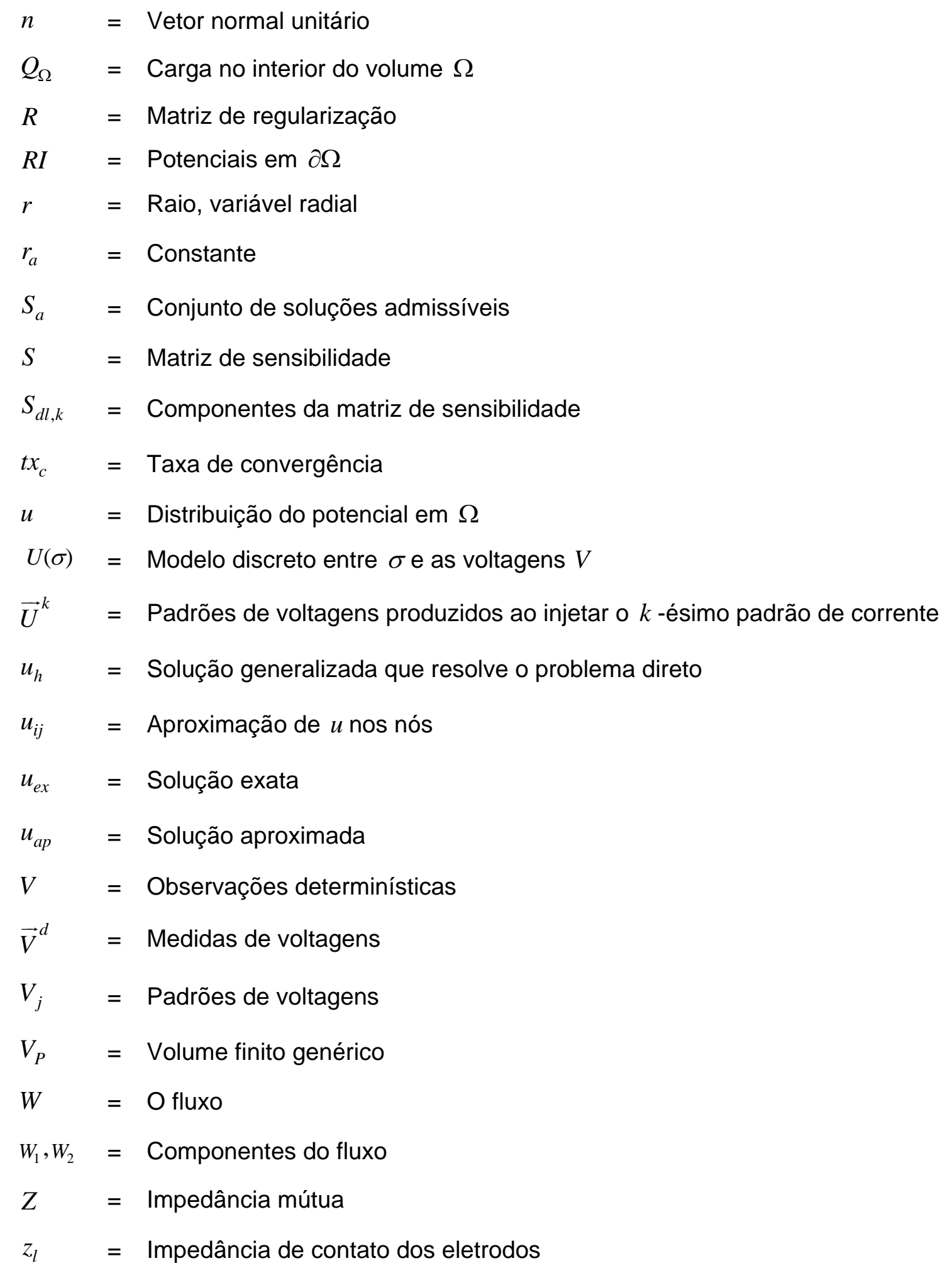




\section{Gregos}

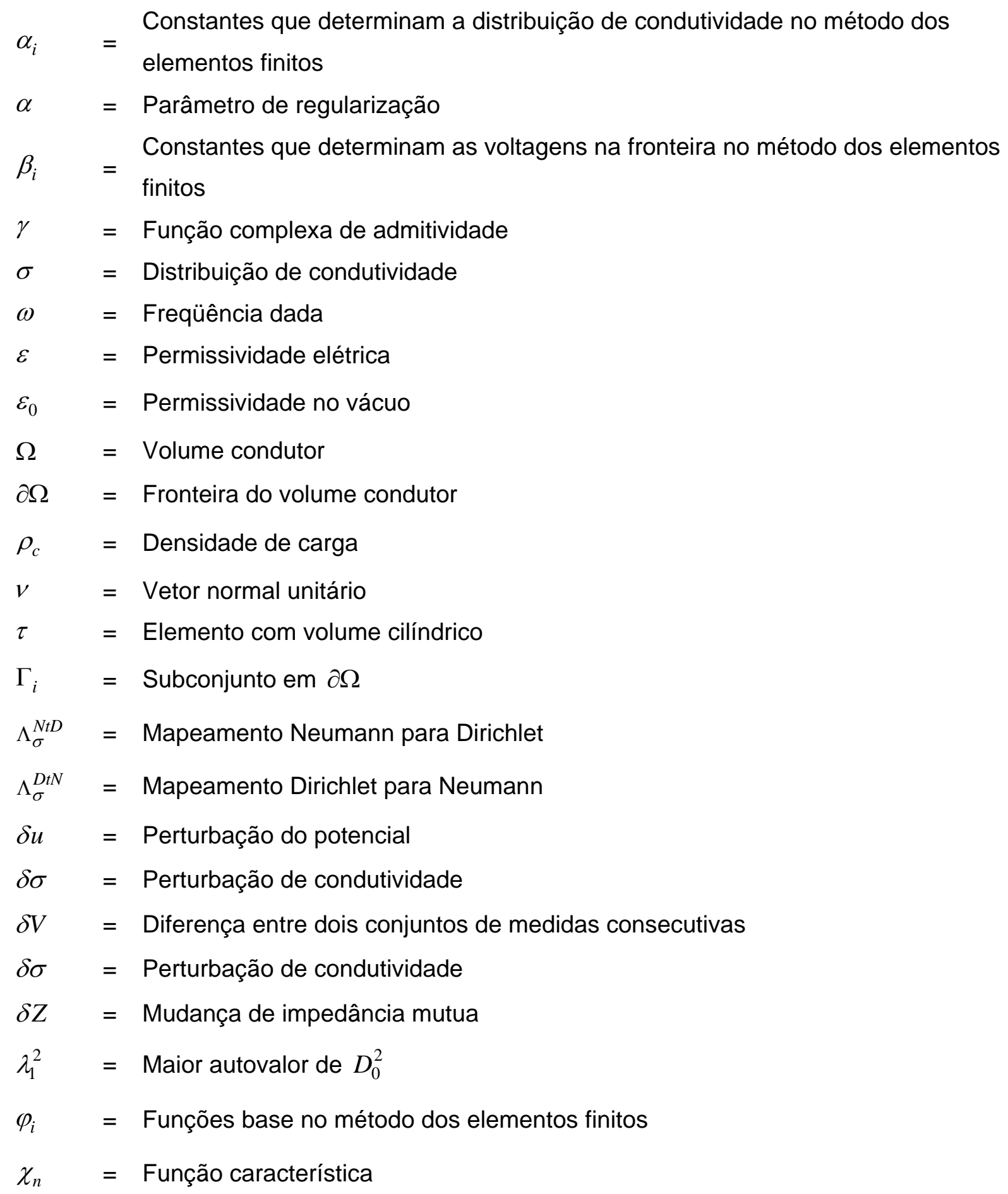




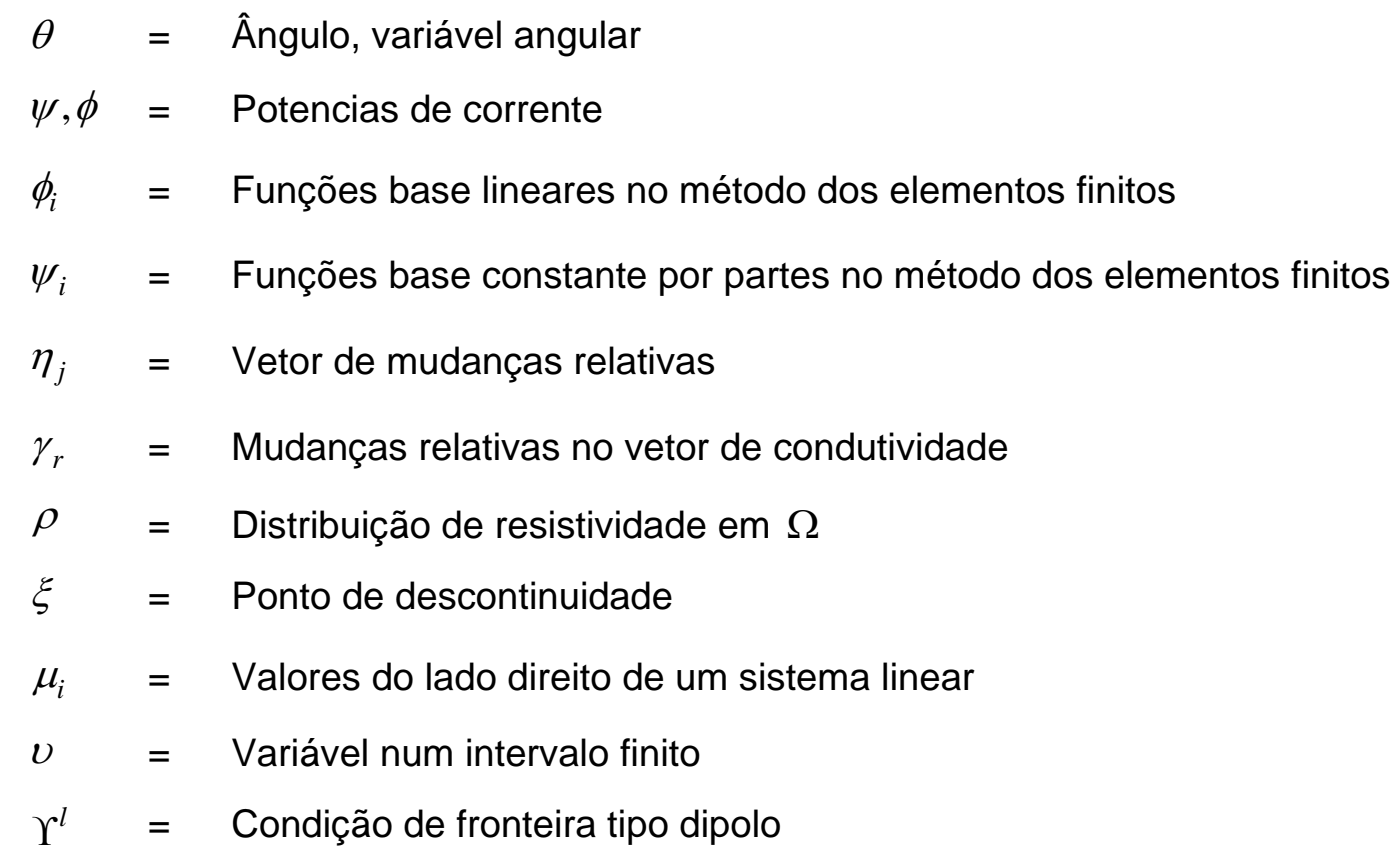




\section{LISTA DE FIGURAS}

Figura 1.1 Sistema TIE aplicado à medicina (Cheney. M, Isaacson. D, Newell. $\mathrm{J}, 1999)$

Figura 1.2 Sistema TIE num processo bifásico (A. Hunt, J. Pendleton, Y. Ladam, 2004)

Figura 2.1 A corrente é injetada no interior de $\Omega$ através dos eletrodos A-B e mede-se a voltagem $\mathrm{V}$ através dos eletrodos C-D.

Figura 3.1 (a) Malha para resolver o problema inverso, (b) Malha para resolver o problema direto.

Figura 3.2 Distribuição de resistividade.

Figura 3.3 Gráfico da curva - $L$

Figura 3.4 A distribuição de resistividade e a aproximação após a $5^{\mathrm{a}}$ iteração.

Figura 4.1 Subdivisão do eixo radial considerando a condição Dirichlet.

Figura 4.2 Subdivisão do eixo radial considerando a condição de Neumann.....

Figura 4.3 Subdivisão do eixo angular.

Figura 4.4 (a) Gráfico da solução exata, (b) Gráfico de $\sigma(r, \theta)$

Figura 4.5

(a) Gráfico da solução exata, (b) Gráfico de $\sigma(r, \theta)$

Figura 4.6 Subdivisão do intervalo $[0,1]$

Figura 4.7 Gráfico da solução exata do Problema (3).....

Figura 4.8 Gráfico do erro relativo na norma do máximo no método HIA \& subdivisões no eixo radial no Problema (3).

Figura 4.9 Gráfico do erro relativo na norma L2 no método HIA \& subdivisões no eixo radial no Problema (3).....

Figura 4.10 Volume finito em coordenadas cartesianas.

Figura 4.11 Domínio [0,1]x[0,1] e a localização da interface.

Figura 4.12 Gráfico da solução exata do Problema (4).

Figura 4.13 Um volume finito em coordenas polares

Figura 4.14 (a) Domínio físico, (b) Domínio computacional. 
Figura 4.15 Malha perto do pólo.

Figura 4.16 (a) Gráfico da solução exata do Problema (5), (b) Interface no disco unitário

Figura 4.17 (a) Gráfico da solução exata do Problema (6), (b) Interfaces no disco unitário.

Figura 4.18 Extrapolação na fronteira.

Figura 5.1 (a) Malhado com volumes finitos no disco unitário quando usamos 16 subdivisões no eixo radial e 32 subdivisões no eixo angular, (b)Posição dos 16 eletrodos na fronteira.

Figura 5.2 Distribuição do potencial devido á injeção de corrente adjacente:

(a)1-padrão (b) 2-padrão, (c)10-padrão (d)15-padrão e (e)16 padrão

Figura 5.3 Distribuição do potencial devido á injeção de corrente pula 1: (a)1padrão (b) 2-padrão, (c)10-padrão (d)15-padrão e (e)16 - padrão....

Figura 5.4 Volume finito $V_{i j}$ correspondente á variável de condutividade $\sigma_{i j} \ldots \ldots$

Figura 5.5 Erro máximo do fluxo na direção radial.

Figura 5.6
(a) Distribuição da condutividade
(b) Reconstrução usando $R=I$,
(c) Reconstrução usando $R \neq I$. 


\section{LISTA DE TABELAS}

Tabela 2.1 Valores de resistividade em tecidos biológicos $[1] \ldots \ldots \ldots \ldots \ldots \ldots \ldots \ldots . . . . . . . . \quad 8$

Tabela 2.2 Valores de resistividade em rochas e fluidos [33]....................... 9

Tabela 4.1 Aproximação do Problema (1) com condição de fronteira tipo Dirichlet e usando fatorização de Choleski.

Tabela 4.2 Taxa de convergência do Problema (1), considerando o erro relativo na norma L2

Tabela 4.3 Aproximação do Problema (1) com condição de fronteira tipo Dirichlet e usando PGC com pré-condicionamento Choleski Incompleto.

Tabela 4.4 Aproximação do Problema (1) com condição de fronteira tipo Dirichlet usando PGC com pré-condicionamento Choleski Incompleto com drop tolerance.

Tabela 4.5 Taxa de convergência ao aplicar PGC com précondicionamento considerando o erro relativo na norma $\mathrm{L} 2 \ldots . . . .$.

Tabela 4.6 Aproximação do Problema (2) com condição de fronteira tipo Dirichlet e usando fatorização de Choleski....

Tabela 4.7 Taxa de convergência do Problema (2) respeito do erro relativo na norma L2.

Tabela 4.8 Aproximação do Problema (2) usando PGC com précondicionamento Choleski Incompleto....

Tabela 4.9 Aproximação do Problema (2) usando PGC com précondicionamento Choleski Incompleto com drop tolerance..........

Tabela 4.10 Taxa de convergência ao aplicar PGC com précondicionamento.

Tabela 4.11 Aproximação do Problema (1) com condição tipo Neumann usando PGC com pré-condicionamento Choleski Incompleto com drop tolerance.

Tabela 4.12 Taxa de convergência do problema (1) com condição de Neumann

Tabela 4.13 Aproximação do Problema (2) com condição tipo Neumann usando PGC com pré-condicionamento Choleski Incompleto com drop tolerance. 
Tabela 4.14 Taxa de convergência do problema (2) com condição de Neumann.

Tabela 4.15 Aproximação aplicando os métodos AA, HA e HIA do Problema 3 usando o erro relativo na norma do máximo.

Tabela 4.16 Aproximação aplicando os métodos AA, HA e HIA do Problema 3 usando o erro relativo na norma $L^{2}$

Tabela 4.17 Aproximação aplicando os métodos HA e HIA do Problema (4) usando o erro relativo na norma do máximo.

Tabela 4.18 Aproximação aplicando os métodos HA e HIA do Problema (4) usando o erro relativo na norma do máximo.

Tabela 4.19 Aproximação aplicando os métodos HA e HIA do Problema (4) usando o erro relativo na norma do máximo com drop tolerance.

Tabela 4.20 Aproximação aplicando o método HIA do Problema (4) usando o erro relativo na norma do máximo e condição de fronteira tipo Neumann com drop tolerance.

Tabela 4.21 Aproximação do problema 5.1 usando o método HA e fatorização de Choleski para resolver o sistema linear......

Tabela 4.22 Aproximação do problema 5.1 com condição de fronteira tipo Neumann usando o método HA e fatorização de Choleski para resolver o sistema linear.

Tabela 4.23 Aproximação do problema 5.1, onde a interface se encontra em 0.5 usando os métodos HA, HIA e eliminação gaussiana para resolver o sistema linear.

Tabela 4.24 Aproximação do problema 5.2 usando o método HA e fatorização de Choleski para resolver o sistema linear.

Tabela 4.25 Aproximação do problema 5.2 com condição de fronteira tipo Neumann usando o método HA e fatorização de Choleski para resolver o sistema linear....

Tabela 4.26 Aproximação do problema 5.2, onde a interface se encontra em 0.5 usando os métodos HÁ, HIA e eliminação gaussiana para resolver o sistema linear.

Tabela 4.27 Aproximações do problema 6.1, usando os métodos HA, HIA e eliminação gaussiana para resolver o sistema linear....

Tabela 4.28 Aproximações do problema 6.2, usando os métodos HA , HIA e eliminação gaussiana para resolver o sistema linear. 
Tabela $4.29 \quad$ Extrapolação na fronteira do problema (1)................................ 85

Tabela $4.30 \quad$ Extrapolação na fronteira do problema (2)................................. 85

Tabela 4.31 Extrapolação na fronteira do problema (4) usando o método

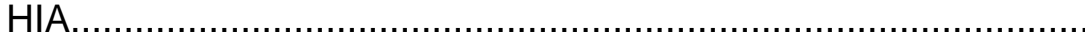

Tabela 4.32 Extrapolação na fronteira do problema 5.2, usando o método HIA

Tabela 5.1 Erro das voltagens nos eletrodos quando usamos normalização e voltagem de referencia na solução do sistema linear (5.1) e injeção adjacente

Tabela 5.2 Erro das voltagens nos eletrodos quando usamos normalização e voltagem de referencia na solução do sistema linear (5.1) e injeção pula1.

Tabela 5.3 Erro do fluxo na direção radial do problema 5.2 usando o

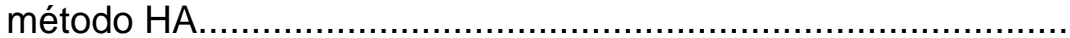

Tabela 5.4 Tempo computacional no cálculo do Jacobiano 


\section{INTRODUÇÃO}

\subsection{Descrição do Problema}

A tomografia por impedância elétrica (TIE) pertence a uma família de imagens eletromagnéticas das quais também fazem parte técnicas como tomografia por capacitância elétrica (TCE)[34], tomografia eletromagnética (TEM)[12] e indução tomográfica (ITM)[6].

Na TIE ([2], [10]) um arranjo de eletrodos é alocado na fronteira de um objeto e uma fonte injeta correntes alternadas (de baixa freqüência) através dos eletrodos e medem-se as voltagens resultantes na fronteira. Com estes dados estima-se a condutividade (ou resistividade) da seção transversal do objeto (dimensão 2) criando-se uma imagem do mesmo. Também é possível obter a reconstrução tridimensional do interior do objeto.

Um sistema típico de TIE é um conjunto de equipamentos que consiste numa unidade de aquisição de dados, uma fonte de corrente, sensores e um computador, neste ultimo fica o código de reconstrução da imagem. Nas figuras (1.1) e (1.2) podemos observar um sistema de TIE sendo aplicado na medicina e em processos industriais.

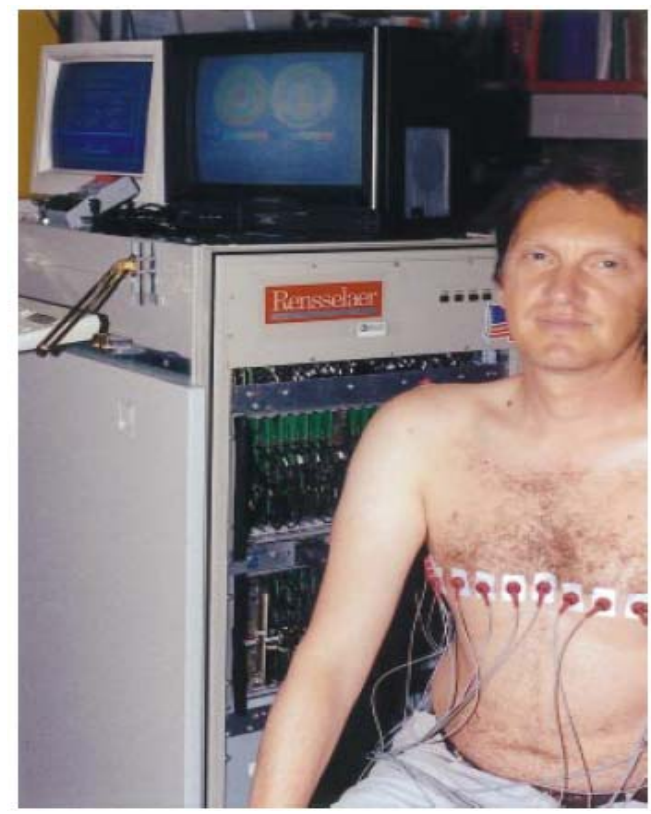

Figura 1.1 Sistema TIE aplicado à medicina (Cheney. M, Isaacson. D, Newell. J, 1999). 


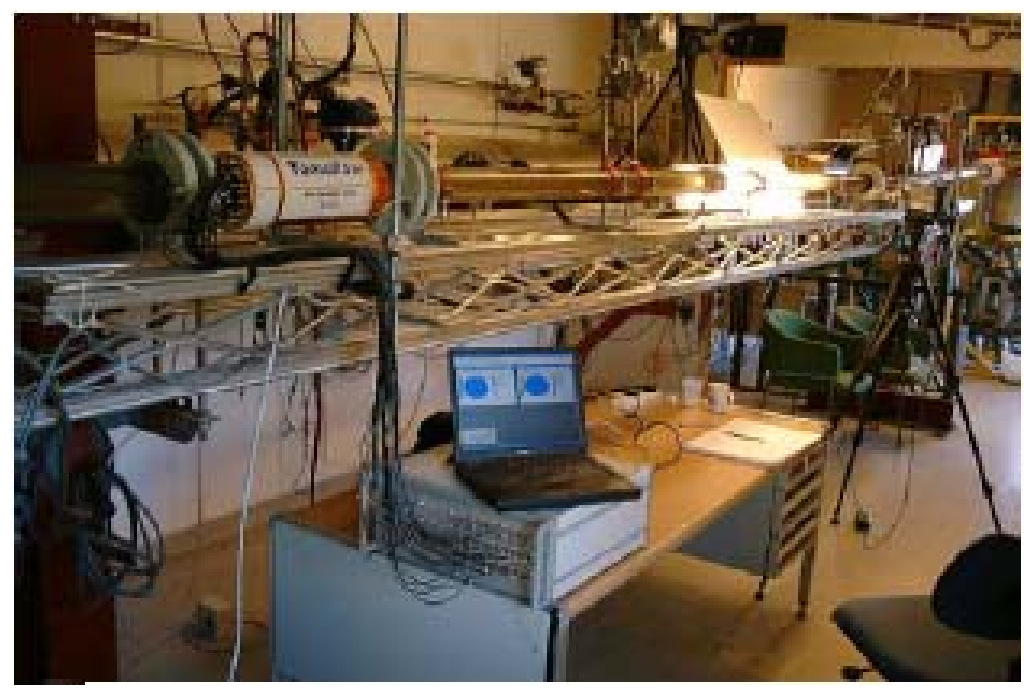

Figura 1.2 Sistema TIE num processo bifásico (A. Hunt, J. Pendleton, Y. Ladam, 2004).

Nas aplicações médicas a técnica TIE é diferente quando comparado com a técnica de tomografia computadorizada (TC) por raios $\mathrm{X}$ a qual é bem conhecida por proporcionar imagens com alta resolução [39]. Na TC o paciente fica no interior de um anel de aproximadamente $70 \mathrm{~cm}$ de diâmetro e na volta deste encontra-se uma ampola de raios X. Durante o processo de scanning uma fonte injeta linhas paralelas de raios $\mathrm{X}$ os quais são guiados dentro do corpo e seguidamente avaliados por detectores de medidas da variação das linhas que emergem do interior do objeto. Em máquinas seqüenciais de terceira geração este procedimento é repetido até fazer uma volta completa $\left(360^{\circ}\right)$ em torno do paciente e em máquinas de quarta geração os equipamentos descrevem uma hélice, em vez de uma sucessão de círculos. Do outro lado da empola de raios $\mathrm{X}$ os detectores de radiação enviam as informações de variação de medidas dos raios $\mathrm{X}$ guiados para um processador o qual desenvolve imagens da variação de distribuição usando algoritmos de reconstrução. Em termos de rapidez computacional e resolução espacial da imagem este método é extremamente eficiente. Isto é principalmente por que os raios $\mathrm{X}$ se propagam em linha reta através do objeto. O resultado é um sistema linear, esparso e bem condicionado. Uma desvantagem da técnica da TC é o uso de raios X o qual tem efeitos negativos no corpo humano, sobretudo pelo risco de causar mutações genéticas em células que estão a se multiplicar rapidamente. Por isso não é recomendável o seu uso em grávidas e em crianças.

A TIE usa a equação elíptica para descrever o campo elétrico, como esse campo é modificado através do objeto as medidas dos valores do potencial no contorno compõem uma 
função não linear da distribuição dos parâmetros elétricos através do objeto. Isso associa um problema de reconstrução que não é fácil de resolver.

Como vantagens da técnica TIE quando comparada com TC podemos salientar o baixo custo e o fato de não ser um método invasivo e a principal desvantagem deste método pode ser atribuída à baixa resolução espacial da imagem reconstruída.

A reconstrução da imagem pode ser vista como uma fonte de informações sobre as propriedades elétricas do interior de um objeto. Nesse contexto uma imagem de baixa qualidade pode limitar as informações que poderiam ser aproveitadas. Assim o melhoramento na resolução da imagem é um dos objetivos principais dos pesquisadores. Dependendo da área onde esta técnica vai ser aplicada temos um ótimo custo-benefício na reconstrução da imagem em um dado volume.

A propriedade de proporcionar uma imagem do interior de um objeto converte a TIE numa ferramenta com importantes aplicações em diferentes áreas como medicina, geofísica, ciências ambientais e em testes não destrutivos de materiais. Em medicina podemos destacar: detecção de êmbolos nos pulmões [15], monitoramento de apnéia [55], monitoramento da função esofágica e gastrointestinal [13], hipotermia [24], monitoramento do fluxo sanguíneo do coração [40], detecção de câncer [69]. Em geofísica e em ciências ambientais a TIE é útil para a localização de depósitos minerais [41], detecção de vazamentos em tanques de armazenagem subterrâneos [36]. Em testes não destrutivos a TIE é aplicada na detecção de corrosão e pequenos defeitos como fissuras ou vazios em metais [2].

\subsection{Revisão Bibliográfica}

Os trabalhos iniciais em imagens médicas apareceram nos inícios de 1980 [4]. Nesse começo se tinha muitos limites computacionais com processadores lentos e um limitado número de ferramentas de medição. Talvez o método mais representativo seja o método linear back-projection introduzido por Barber e Brown [3], [4], [5]. Naquela época a modelagem dos eletrodos não era considerada como um ponto crucial do método. Posteriormente os pesquisadores perceberam a necessidade de compensar uma boa 
modelagem de eletrodos na diminuição dos ruídos nas medidas. Pidcock et al. [43] e Hua et al [18] adotaram uma técnica de medição chamada de "quatro eletrodos" atualmente essa técnica é usada em medidas de bioimpedância. Em particular Hua e co-autores deram uma solução prática em forma de eletrodos compostos onde eles usavam diferentes partes de um mesmo eletrodo para propósito de excitação e medição. Este método foi amadurecendo ate o chamado "método dos quatro eletrodos" de Barber e Brown [5] e posteriormente foi implementado nos sistemas de adquisição de dados: OXBACT II / III e no Mark III desenvolvidos na universidade de Sheffield. Atualmente as modelagens dos eletrodos estão mais sofisticadas e realistas e o "Modelo Completo de Eletrodos (MCE)” foi convertido numa técnica quase que padrão.

Apesar de que a comunidade de médicos e engenheiros priorizava esforços com o hardware e na busca de aplicações da TIE em diferentes áreas, os pesquisadores em matemática se concentravam nos aspectos teóricos do problema. A grande questão da comunidade era se um conjunto de medidas em particular poderia determinar de forma única uma distribuição de condutividade no interior de um corpo. Um primeiro intento para responder esta questão foi dado por Calderón em 1980 [8], onde ele mostra que a derivada Fréchet do operador direto era uma função injetora em algum espaço normado e assim uma única inversa poderia ser calculada. Em 1992, Somersalo, Cheney e Isaacson [53], mostraram a existência e unicidade da solução do problema direto para o caso bidimensional usando o MCE, similares estudos foram feitos por Konh e Vogelius [28], Sylvester e Uhlmann [49] mostraram a existência e unicidade em modelos tridimensionais com condições de contorno simples. Atualmente, Murai [39], Breckon[7] e Pidcock[43] tem publicado algoritmos de reconstrução baseados no método SVD truncado. Breckon, explicitamente prova que o problema linear e sua linearização são mal postos em termos da decomposição de valores singulares, conduzindo o problema no caminho da programação não linear e no uso de técnicas de regularização. O mesmo autor é pioneiro na pesquisa sobre a existência e unicidade da solução do problema inverso em domínios anisotrópicos.

Os esforços relacionados com a busca de qualidade nas imagens reconstruídas conduziram na busca de padrões de corrente ótimas para substituir os convencionais padrões de corrente conhecidos como “adjacente” e “oposto”. Nos artigos [22], [23] Isaacson mostra que padrões trigonométricos de corrente, usados para maximizar a distinguibilidade de uma distribuição em particular, produziam ótimos resultados no modelo dos quatro eletrodos. 
Mais recentemente em 1997 Vaukhonen M. [57] explica a importância de se ter informações a priori para estabilizar a solução inversa. Suas pesquisas incluem também a estimação dinâmica da condutividade, a aproximação bayesiana assim como os métodos de bases condicionadas e regularização por subespaços. Posteriormente estas técnicas foram adaptadas em problemas tridimensionais no grupo de tomografia por impedância elétrica aplicada ao diagnóstico médico da Universidade de Kuopio.

Dispõe-se em forma de códigos abertos o projeto de software "Electrical Impedance and Diffuse Optical Reconstruction Software (EIDORS)”. Esses códigos resolvem o problema direto e inverso em problemas de admitividade em duas e três dimensões [60]. Similarmente, existe um software em problemas de tomografia aplicado em óptica chamado TOAST Project (http://www.ucl.ac.uk/MedPhys/toast/index.htm), [48].

A maioria dos problemas em imagens médicas e industriais é tridimensional, com exceção dos problemas que são invariantes numa direção espacial. Alguns estudos em 3D foram feitos por Kleinermann [27] e Pinheiro [44] com resultados interessantes. Recentemente foram publicados códigos abertos em 3D desenvolvido na UMIST [1].

\subsection{O Problema Inverso de Condutividade é mal posto}

Resolver o problema direto é achar um único efeito, dada uma ou várias causas, usando apropriados modelos físicos. Esses problemas geralmente são bem postos os quais conduzem a uma única solução a qual não é afetada por pequenas mudanças nos dados iniciais. Atualmente é sabido que na ciência e tecnologia se lidam com problemas que não são bem postos. Tais problemas têm um objetivo inverso ao do problema direto e podem ser interpretados como achar a causa de um dado efeito ou achar uma lei física que cause o efeito. Esses problemas, geralmente, não têm uma única solução.

Em 1932 Hadamard [16] descreveu propriedades genéricas de problemas que provêm de fenômenos físicos. Para um dado conjunto de observações podemos mapear unicamente para um dado conjunto de soluções. Assim, de acordo com Hadamard, um problema é bem posto se para um dado conjunto de observações:

1. Existe uma solução que é consistente com as observações. 
2. A solução é única.

3. A solução depende continuamente das observações.

Em outras palavras, dada uma função $F: D_{a} \rightarrow S_{a}$, onde $D_{a}$ é um conjunto de dados admissíveis e $S_{a}$ é o conjunto de soluções admissíveis então existe $F^{-1}$ contínua, assim para uma solução com a propriedade de boa resolução espacial $E_{S}$, existe um $E_{D}$ ótimo para o qual $E_{S}$ é alcançado para o nível de ruído dado. Chamamos de problema mal posto segundo Hadamard se alguma das três condições não é satisfeita. O problema inverso de condutividade é mal posto no sentido de Hadamard apesar de que, condições naturalmente impostas, podem assegurar a validade das primeiras duas condições, estas certamente falham na terceira condição pois pequenas perturbações nas medidas podem implicar em grandes oscilações na solução.

Quando aproximamos um problema mal posto, geralmente, a tendência é transformálo num problema equivalente com a propriedade de ser menos mal posto. Do ponto de vista prático, no problema inverso de condutividade trabalha-se com dados incompletos, pois não é possível injetar padrões de corrente arbitrários na fronteira do objeto. Assim, a transformação pode ser feita por vários caminhos um deles é a regularização.

Regularização é essencialmente proporcionar ao problema inverso informação a priori acerca da solução, nesse sentido podemos dizer que é uma condição de preservar a existência, unicidade e também a estabilidade da solução. Desde outra perspectiva, técnicas de regularização podem ser interpretadas como um balanço entre as condições a priori e a influência das medidas na solução.

\subsection{Objetivos}

A modelagem do problema direto na TIE leva à solução numérica da equação de difusão com coeficientes variáveis, não homogêneos. O problema direto tem que ser resolvido eficientemente, pois ele é “chamado” freqüentemente pelo método inverso.

Apesar de que existem técnicas conhecidas para resolver o problema como o dado em [29],[36],[37],[48] aplicações de médio e grande porte precisa de um método cada vez mais 
rápido e eficiente, em particular, o caso onde os coeficientes variáveis têm a característica de ser descontínuos ainda merece atenção [14],[20],[51],[68].

O presente trabalho tem como objetivo principal apresentar a formulação e solução numérica do problema direto usando métodos como diferenças finitas e volumes finitos [14], [48]. Para isso estudaremos a solução numérica do problema de difusão em domínios retangulares e polares e os coeficientes de difusão como sendo contínuos e descontínuos. Os métodos numéricos propostos serão testados em problemas onde a solução analítica é conhecida para posteriormente serem aplicados na solução numérica do problema direto que trata a TIE.

Outro objetivo a ser apresentado é propor a maneira como o fluxo é aproximado em cada nó, em vista de propor um método para a aproximação do Jacobiano (ou matriz de sensibilidade) o qual é um ponto crucial na técnica de reconstrução de imagens que usa a metodologia da tomografia por impedância elétrica.

\subsection{Divisão da tese}

O presente trabalho se divide em seis capítulos.

O capítulo 1 trata da introdução do problema e uma revisão bibliográfica dos trabalhos relacionados.

No capítulo 2 fazemos a formalização do problema e os fundamentos teóricos que usa o presente trabalho.

O capítulo 3 trata da aplicação da metodologia da TIE usando os algoritmos dados no EIDORS.

No capítulo 4 resolvemos o problema direto com diferentes métodos numéricos e para diferentes formas da condutividade.

No capitulo 5 aplicamos os métodos desenvolvidos no capitulo 4 para resolver o problema direto e descrevemos uma maneira de aproximar o Jacobiano.

Por último, no capítulo 6 são dadas as conclusões e recomendações do trabalho. 


\section{FUNDAMENTOS TEÓRICOS}

\subsection{Equações governantes}

Consideramos um domínio limitado e simplesmente conexo $\Omega \subset \mathfrak{R}^{n}, n \geq 2$. A função complexa $\gamma$ de admitividade definida num ponto $x \in \Omega$ e numa freqüência $\omega$ é definida como:

$$
\gamma(x, \omega)=\sigma(x)+i \omega \varepsilon(x), \quad i=\sqrt{-1},
$$

onde $\sigma$ é a condutividade e $\varepsilon$ é a permissividade elétrica.

A condutividade e a permissividade elétrica determinam o comportamento dos materiais sob a influência de campos elétricos externos. A impedância elétrica é a inversa da admitividade e mede a razão entre a tensão elétrica e a corrente elétrica num ponto $x \in \Omega$.

A tomografia por impedância elétrica é um problema inverso que determina a distribuição da condutividade ou resistividade no interior de $\Omega$ dados que simultaneamente correntes são injetadas e voltagens são medidas em $\partial \Omega$.

Dado que os materiais possuem diferentes propriedades elétricas então uma imagem da distribuição de $\sigma$ ou $\varepsilon$ pode inferir em conhecer a estrutura interna do interior de $\Omega$. Assim devido a esta propriedade a TIE pode ser aplicada em diferentes áreas.

Na tabela 2.1 podem ser observados diferentes valores de resistividade em tecidos biológicos e na tabela 2.2 a resistividade em rochas e fluidos.

Tabela 2.1 Valores de resistividade em tecidos biológicos [2].

\begin{tabular}{|c|c|c|}
\hline Tecidos & $1 / \sigma(\Omega \mathrm{cm})$ & $\varepsilon\left(\mu \mathrm{Fm}^{-1}\right)$ \\
\hline Pulmão & 950 & 0.22 \\
\hline Músculo & 760 & 0.49 \\
\hline Fígado & 685 & 0.49 \\
\hline Coração & 600 & 0.88 \\
\hline Tecido adiposo & $>1000$ & 0.18 \\
\hline
\end{tabular}


Tabela 2.2 Valores de resistividade em rochas e fluidos [38].

\begin{tabular}{|l|l|}
\hline Rochas ou Fluidos & $1 / \sigma(\Omega \mathrm{cm})$ \\
\hline Areia marina, xisto & $1-10$ \\
\hline Areia terrestre & $15-50$ \\
\hline Rocha vulcânica, basalto & $10-200$ \\
\hline Granito & $500-2000$ \\
\hline Dolomita & $50-5000$ \\
\hline Água clorada & 0.16 \\
\hline Água sulfurada & 1.2 \\
\hline
\end{tabular}

Na TIE aspectos importantes na modelagem matemática é a dimensão, geometria da fronteira e características estruturais do volume físico. Além disso, a localização dos eletrodos na fronteira tem que ser tratada convenientemente para ter observações de boa qualidade. Assim, as características da modelagem têm que conduzir em uma simulação realista da distribuição do potencial e o modelo têm que refletir a locação dos eletrodos e a impedância de contato.

\subsection{O Problema Direto}

As inter-relações que governam as interações da eletricidade e magnetismo num problema físico são resumidas pelas equações de estado de Maxwell.

Para um volume isotrópico de condutividade $\Omega$, onde o contorno $\partial \Omega$ é de classe $C^{2}$ as equações das relações elétricas são:

$$
\begin{aligned}
& \nabla . E=\frac{\rho_{c}}{\varepsilon_{0}} \\
& \nabla \times E=0,
\end{aligned}
$$

e as equações das relações magnéticas são:

$$
\begin{gathered}
\nabla . B=0 \\
\nabla \times B=\frac{j}{\varepsilon_{0}},
\end{gathered}
$$


onde $E$ é um campo elétrico, $B$ é a densidade de fluxo magnético, $\rho_{c}$ a densidade de carga, $j$ a densidade de corrente e $\varepsilon_{0}$ uma constante.

Da teoria do cálculo quando $\nabla \cdot E=0$ então existe um escalar $u$ cujo gradiente é igual ao vetor, então de (2.2):

$$
E=-\nabla u
$$

A densidade de corrente $j$ pode ser assumida como invariante no tempo. Se $I^{d}$ é o $d$-ésimo padrão de corrente desde um eletrodo $s$, então:

$$
I^{d}=\int_{s} j . v d s
$$

Das leis de conservação de carga, se $l$ é a superfície de $\partial \Omega$ então:

$$
\int_{l} j . d l=-\frac{d}{d t}\left(Q_{\Omega}\right) .
$$

A carga no interior do volume $Q_{\Omega}$ pode ser expressa como o volume integral da densidade de carga, assim:

$$
Q_{\Omega}=\int_{\Omega} \rho_{c} d V
$$

Combinando (2.7) e (2.8) temos:

$$
\nabla \cdot j=-\frac{\partial \rho_{c}}{\partial t}
$$

O qual basicamente é outra maneira de expressar o teorema de conservação de carga. Como não há uma fonte de corrente no interior do volume e sabendo que $E$ não muda no tempo, o lado direito de (2.9) tem que ser zero: 


$$
\nabla \cdot j=0 \text {. }
$$

Num meio isotrópico linear a densidade de corrente e o campo elétrico são relacionados pela seguinte aproximação:

$$
j \approx \gamma E \approx(\sigma+i \omega \varepsilon) E
$$

onde $\sigma$ é a condutividade elétrica, $\varepsilon$ é a permissividade elétrica e $\gamma$ é a admitividade complexa do meio. Considerando aparte real de $\gamma$ e substituindo (2.5) e (2.11) em (2.10) temos:

$$
\nabla . \sigma E=0
$$

Agora, combinado esta última expressão com (2.5) temos a seguinte equação diferencial elíptica:

$$
\nabla . \sigma \nabla u=0
$$

Assim, a equação diferencial elíptica de segunda ordem (2.13) conhecida como equação de difusão com coeficientes variáveis rege a distribuição do potencial eletrostático $u$ no meio condutivo $\Omega$.

Para derivar as condições de fronteira que acompanham a equação (2.13) consideremos um elemento $\tau$ com volume cilíndrico na superfície de $\Omega$. Integrando a equação $\nabla \cdot \sigma E=-\nabla \cdot J^{s}$, onde $J^{s}$ é uma fonte de corrente, sobre o volume $\tau$ :

$$
\int_{\tau} \nabla \cdot \sigma E d \tau=-\int_{\tau} \nabla \cdot J^{s} d \tau
$$

e usando o teorema da divergência:

$$
\int_{S_{\tau}} \sigma E . v d S_{\tau}=-\int_{S_{\tau}} J^{s} \cdot v d S_{\tau}
$$


onde $S_{\tau}$ é a fronteira de $\tau$ e $v$ é o vetor normal unitário.

Sabendo que $J^{S}=0$ dentro de $\tau$ e fora dele se cumpre que $E=0$, temos as relações:

$$
-\left.\sigma E \cdot v\right|_{\text {in }}=-\left.J^{s} \cdot v\right|_{\text {out }}
$$

usando a relação (2.5) concluímos que:

$$
\sigma \frac{\partial u}{\partial v}=-J^{s} \cdot v=j
$$

onde $j$ é componente normal, negativa da densidade de corrente elétrica injetada $J^{s}$. Daqui em diante denotaremos como $j$ a corrente elétrica injetada.

O modelo contínuo da TIE assume que não há eletrodos. Assim, a corrente elétrica injetada é uma função contínua:

$$
j(\zeta)=C_{0} \cos (k \zeta)
$$

onde $C_{0}$ é uma constante. Para este modelo em [9] é mostrado que a resistividade é sobreestimada em mais de 25\%, pois os efeitos dos eletrodos são ignorados.

Soluções analíticas para o modelo contínuo podem ser encontradas em [49], [53].

Outros modelos onde os efeitos dos eletrodos são levados em conta são:

O modelo “gap" [2] assume que a corrente injetada $j$ satisfaz:

$$
j=\left\{\begin{array}{l}
\frac{I_{l}}{\left|e_{l}\right|}, x \in e_{l}, l=1,2, . ., L \\
0, x \in \partial \Omega \backslash \bigcup_{l=1}^{L} e_{l}
\end{array},\right.
$$


onde $\left|e_{l}\right|$ é a área do eletrodo, $I_{l}$ é a corrente injetada no $l$-ésimo eletrodo e $L$ o número total de eletrodos. Este modelo ainda que leve em conta os eletrodos na fronteira ainda sobreestima a resistividade. Modelos mais acurados levam em conta a impedância de contato do eletrodo e o volume condutor.

O modelo "shunt" [2] leva em conta a propriedade do potencial elétrico no eletrodo ser uma constante. Assim a relação (2.18) é substituída pela condição:

$$
\int_{e_{l}} \sigma \frac{\partial u}{\partial v} d S=I_{l,} x \in e_{l}, l=1,2, . ., L
$$

e o efeito shunt é considerado em:

$$
u=U_{l}, x \in e_{l}, l=1,2 . ., L
$$

onde $U_{l}$ é a voltagem medida no $l$-ésimo eletrodo. Segundo [59] este modelo ainda não estima a resistividade corretamente, pois a impedância de contato é ignorada.

As seguintes condições de contorno acompanham a equação (2.13) e são conhecidas como o modelo completo de eletrodos (MCE) [1], [5]:

Se $\Gamma_{1} \subset \partial \Omega$ é o subconjunto da superfície do volume sob os eletrodos, então a densidade de corrente associada é:

$$
\sigma \frac{\partial u}{\partial v}=j \text { em } \Gamma_{1}
$$

para o restante da superfície $\Gamma_{2}=\partial \Omega \backslash \Gamma_{1}$,

$$
\sigma \frac{\partial u}{\partial v}=0 \text { em } \Gamma_{2}
$$


Para cada eletrodo a integral de densidade de corrente sobre a superfície do eletrodo $s$ é igual à quantidade de corrente desse eletrodo,

$$
\int_{e_{l}} \sigma \frac{\partial u}{\partial v} d s=I_{l}, x \in e_{l}, l=1, \ldots, L,
$$

onde $L$ é o número de eletrodos do sistema. Os valores do potencial $U_{l}$, os quais são medidos no $l$-ésimo eletrodo, são iguais à soma dos potenciais sobre a superfície desses eletrodos e os potenciais que ocorre pela impedância de contato dos eletrodos $z_{l}\left[\Omega / m^{2}\right]$,

$$
u+z_{l} \sigma \frac{\partial u}{\partial v}=U_{l}, x \in e_{l}, l=1,2, \ldots, L
$$

Este modelo tem uma única solução [53], quando consideramos o teorema de conservação de carga:

$$
\int_{\partial \Omega} j=0 \Leftrightarrow \sum_{l=1}^{L} I_{l}=0,
$$

e para as medidas fazemos a seguinte escolha:

$$
\int_{\partial \Omega} u=0 \Leftrightarrow \sum_{l=1}^{L} U_{l}=0 .
$$

O problema direto em TIE pode ser formulado da seguinte maneira:

Dada $\sigma$ queremos determinar as voltagens $\left.u\right|_{\partial \Omega}$ para os padrões de corrente $I^{d}$ que satisfazem (2.26), para isso resolvemos a equação (2.13) junto às condições de fronteira descritas acima. Em outras palavras, o problema direto é determinar o mapeamento Neumann para Dirichlet $\Lambda_{\sigma}^{N t D}: H^{-1 / 2}(\partial \Omega) \rightarrow H^{1 / 2}(\partial \Omega)$, definido como:

$$
\Lambda_{\sigma}^{N t D} I^{d}=\left.u\right|_{\partial \Omega}
$$


Uma linearização do problema direto é feita da seguinte maneira. Estamos interessados em mensurar a perturbação $\delta$ em $\partial \Omega$, quando introduzimos uma perturbação de condutividade $\delta \sigma$ em $\Omega(\delta \sigma \ll \sigma)$ então a equação de difusão (2.13) fica:

$$
\nabla \cdot((\sigma+\delta \sigma) \nabla(u+\delta u))=0, \mathrm{em} \Omega
$$

então:

$$
\nabla \cdot(\sigma \nabla u)+\nabla \cdot(\sigma \nabla(\delta u))+\nabla \cdot(\delta \sigma \nabla u)+\nabla \cdot(\delta \sigma \nabla(\delta u))=0
$$

Considerando novamente a equação (2.13) temos que o primeiro termo do lado esquerdo da equação (2.29) é nulo e considerando que os termos $\delta \sigma, \delta u$ são variações pequenas temos o seguinte problema linearizado:

$$
\nabla \cdot(\sigma \nabla(\delta u))=-\nabla\left(\frac{\delta \sigma}{\sigma}\right) \cdot \nabla u
$$

então a condição (2.17) fica :

$$
\sigma \frac{\partial(\delta u)}{\partial v}=-\delta \sigma \frac{\partial u}{\partial v}, \operatorname{em} \partial \Omega
$$

Para resolver o problema direto encontramos na literatura que o método dos elementos finitos é os mais utilizados [32], [45], [57], [58], [23]. Para problemas cuja fronteira seja suficientemente regular podemos aplicar métodos como diferenças finitas [2] ou volumes finitos [69].

Para problemas bi-dimensionais o sistema linear gerado pelo método numérico pode ser resolvido usando métodos diretos como o método de eliminação gaussiana ou usar fatorização da matriz, onde dependendo das propriedades da matriz, podemos usar fatorização de Choleski, fatorização $L U$ entre outros. O sistema linear também pode ser resolvido usando métodos iterativos como os métodos com pré-condicionamento (Choleski incompleto, 
$L U$ incompleto, etc), métodos multigrid. Atualmente implementações que usam o método multigrid e malhas adaptativas estão sendo aplicadas [32].

\subsection{A coleta de dados na TIE}

Para resolver o problema direto precisamos de padrões de corrente elétrica injetadas através dos eletrodos e fazer as medições das voltagens resultantes. A questão de ter medidas razoáveis é um ponto importante na TIE. Para isso muitas estratégias tem sido implementadas [4], [28], [23].

A maioria de sistemas de TIE tem preferido em injetar corrente e medir as voltagens resultantes (em vez de fazer o contrário) devido a que a impedância de contacto entre o eletrodo e o meio condutor não tem efeitos notáveis nas medidas das voltagens, pois, os voltímetros têm uma grande impedância de entrada ao contrário dos ohmímetros. Injetar corrente e medir voltagens caracterizam em resolver o problema (2.13) junto á condição de Neumann. Fazer o contrário implica em impor á condição de Dirichlet.

Existem duas formas principais de coletar dados de entrada. A primeira é chamada método dos dois eletrodos, na qual as voltagens dos eletrodos são medidas dos mesmos eletrodos de onde se injetaram as correntes. A outra forma é o chamado método dos quatro eletrodos onde as correntes são injetadas de alguns eletrodos e as medidas são tomadas dos outros eletrodos. Este método minimiza os erros nas voltagens medidas devido à impedância de contato o qual se apresenta em ambos os métodos de coleta de dados.

Uma forma de aplicar o método dos quatro eletrodos é o chamado método dos eletrodos vizinhos [45], [57], na qual a corrente é injetada desde dois eletrodos adjacentes e as diferencias de voltagem são medidas de todos os outros pares de eletrodos. O método é repetido em todos os eletrodos. Assim, por exemplo, quando temos 16 eletrodos na fronteira do objeto condutor um total de 16x13=208 medidas são feitas. Porém, somente 104 destas medidas são independentes devido ao teorema de reciprocidade, a qual afirma que invertendo as medidas das voltagens e injeção de corrente nos eletrodos obtemos o mesmo valor de condutividade ou resistividade [45]. 
Outra versão do método dos quatro eletrodos é o método oposto na qual as correntes são injetados através de eletrodos diametralmente opostos. As medidas das voltagens são feitas tomando como referência o eletrodo adjacente do eletrodo de injeção de corrente. Este método produz o mesmo numero de medidas independentes do método dos eletrodos vizinhos, porem com a vantagem de produzir uma densidade de corrente mais uniforme.

A combinação dos métodos descritos resulta no método cruzado [45] na qual um eletrodo (de um total de 16 eletrodos), por exemplo, o eletrodo 1 é o referencial de corrente e a fonte de corrente é aplicado sucessivamente nos eletrodos 3,5,7,...,15. Para cada par de corrente injetada as voltagens são medidas com respeito ao eletrodo 2 de todos os eletrodos exceto daquele que serviu de referencia na injeção de corrente. Este método também produz o mesmo numero de medidas independentes que os outros dois métodos anteriores.

Segundo [61] a posição angular ótima de dois eletrodos injetores de corrente que produz a melhor sensibilidade (diferença de voltagens entre a distribuição homogênea e perturbada) é aproximadamente $48^{\circ}$. Assim em vez de injetar corrente entre eletrodos adjacentes temos que aplicar nos pares: 1-3; 2-4; ...; N-1. Este modelo também é conhecido como pula 1.

A qualidade de imagens tem relação com a forma que os dados são coletados. Em [22] aparece o termo "distinguibilidade" de condutividade e posteriormente é estudado em [32],[33]. Duas condutividades $\sigma_{1}, \sigma_{2}$ são “distinguíveis” por medidas de precisão $\varepsilon$ se existe um padrão de correntes $I,\|I\|=1$ e:

$$
\left\|R\left(\sigma_{1}\right) I-R\left(\sigma_{2}\right) I\right\|^{2}>\varepsilon
$$

onde $R(\sigma) I$ denota os potencias elétricos ou voltagens em $\partial \Omega$ resultantes da aplicação de padrões de correntes $I$ contendo a condutividade $\sigma$.

Assim, para maximizar a “distinguibilidade” $\left\|R\left(\sigma_{1}\right) I-R\left(\sigma_{2}\right) I\right\|^{2}$ com respeito ao padrão de correntes $I$ fazemos: 


$$
\max _{I} \frac{\left\|R\left(\sigma_{1}\right) I-R\left(\sigma_{2}\right) I\right\|^{2}}{\|I\|}=\max _{I} \frac{\left\|D_{0} I\right\|^{2}}{\|I\|}
$$

onde $D_{0}=\left(R\left(\sigma_{1}\right)-R\left(\sigma_{2}\right)\right)$. Assim, temos que o padrão de corrente ótimo que maximiza (2.34) é o autovetor de $D_{0}^{2}$ que tem o maior autovalor. Assim, $\sigma_{1}$ é “distinguível” de $\sigma_{2}$ por medidas de precisão $\varepsilon$ se $\sqrt{\lambda_{1}^{2}}>\varepsilon$, onde $\lambda_{1}^{2}$ é o maior autovalor de $D_{0}^{2}$. O próximo melhor padrão de corrente corresponde aos autovetores que tem o segundo maior autovalor. Todos aqueles autovetores para os quais $\sqrt{\lambda_{k}^{2}}>\varepsilon$ são padrões razoáveis a serem usados na injeção de corrente.

Dada essa definição é possível otimizar o melhor padrão de correntes e usar isso para injetar corrente no volume de modo a ter a melhor "distinguibilidade”. Por exemplo, segundo estudos feitos em [21] os chamados padrões de corrente trigonométricos são a melhor opção de padrões de corrente elétrica para distinguir uma distribuição não homogênea circular dentro de um volume condutor circular.

Os padrões de corrente trigonométricos são dados da seguinte maneira:

$$
I_{l}^{d}=\left\{\begin{array}{ll}
\cos \left(d \varsigma_{l}\right), & l=1, \ldots, L, d=1, \ldots, L / 2 \\
\operatorname{sen}\left((d-L / 2) \varsigma_{l}\right), & l=1, \ldots, L, d=L / 2+1, \ldots, L-1
\end{array},\right.
$$

onde $\varsigma_{l}=\frac{2 \pi l}{L}, d$ é o número de padrões de corrente e $L$ o número de eletrodos alocados em $\partial \Omega$.

\subsection{O Problema Inverso}

O problema inverso em TIE pode ser formulado da seguinte maneira: dada uma distribuição de medidas de voltagens $\left.u\right|_{\partial \Omega}$ para todos os padrões de corrente $I^{d}$, ou, dado conhecido o mapeamento $\Lambda_{\sigma}^{N t D}$ temos que reconstruir $\sigma$. Assim temos que determinar o mapeamento Dirichlet para Neumann $\Lambda_{\sigma}^{D t N}=H^{1 / 2}(\partial \Omega) \rightarrow H^{-1 / 2}(\partial \Omega)$, definido como: 


$$
\left.\Lambda_{\sigma}^{D t N} u\right|_{\partial \Omega}=\sigma \frac{\partial u}{\partial v}
$$

Os métodos de reconstrução em TIE podem ser classificados em dois grandes grupos: determinísticos e probabilísticos alem de outras abordagens como o método $d$-bar [9] backprojection [4], variacionais [28], layer stripping [22], método da perturbação [63], variação total mínima [57], filtro de Kalman [11] entre outros.

Um modelo de observações determinísticas tem a forma:

$$
V=U(\sigma ; I)
$$

onde $V \in \mathfrak{R}^{L P}$, sendo que $L$ é o número de eletrodos e $P$ o número de padrões de corrente usados, $U(\sigma ; I)$ representa o modelo discreto entre os parâmetros $\sigma \in \mathfrak{R}^{K}$ e as observações $V$. Daqui para frente denotaremos $U(\sigma)$ em substituição de $U(\sigma ; I)$.

Como a dependência das observações a respeito de $\sigma$ é não linear, temos que usar algum método iterativo apropriado para obter a solução $\sigma$. Nesses termos o modelo (2.37) pode ser linearizado em torno de uma distribuição de referência $\sigma_{0}$. Assim:

$$
U(\sigma)=U\left(\sigma_{0}\right)+\frac{\partial U}{\partial \sigma}\left(\sigma_{0}\right)\left(\sigma-\sigma_{0}\right)+O\left(\left\|\sigma-\sigma_{0}\right\|^{2}\right)
$$

onde $\frac{\partial U\left(\sigma_{0}\right)}{\partial \sigma} \in \mathfrak{R}^{L P \times K}$ é o Jacobiano de $U(\sigma) \in \mathfrak{R}^{L P}$ em $\sigma=\sigma_{0}$. Desconsiderando os termos de segunda ordem de (2.38) temos:

$$
\delta U \approx J \delta \sigma
$$

onde: $J=\frac{\partial U\left(\sigma_{0}\right)}{\partial \sigma}, \delta U=U(\sigma)-U\left(\sigma_{0}\right)$ e $\delta \sigma=\sigma-\sigma_{0}$.

Assim temos que resolver o seguinte problema de minimização: 


$$
\min _{\delta \sigma}\|\delta V-J \delta \sigma\|
$$

onde $\delta V$ é a diferença entre dois conjuntos de medidas.

Para resolver o problema (2.40) métodos iterativos são usados, principalmente aqueles baseados no algoritmo de Gauss-Newton com parâmetros e matrizes de regularização [19], [22], [33], [60].

Para lidar com o mau condicionamento do Jacobiano são aplicados diversos métodos de regularização para termos uma solução estável. Por exemplo, o método LevenbergMarquardt [30] tem o seguinte processo iterativo:

$$
\sigma_{i+1}=\sigma_{i}+\delta \sigma_{i}
$$

onde:

$$
\delta \sigma_{i}=\left(J_{i}^{T} J_{i}+\alpha I\right)^{-1}\left(J_{i}^{T}\left(V-U\left(\sigma_{i}\right)\right)\right),
$$

sendo $\alpha$ o parâmetro de regularização.

Nos métodos determinísticos é preciso aprimorar o cálculo do Jacobiano o qual tem que ser feito de maneira eficiente, pois no seu cálculo é gasto uma grande parcela do tempo computacional total do problema.

O Jacobiano tem a seguinte forma: 


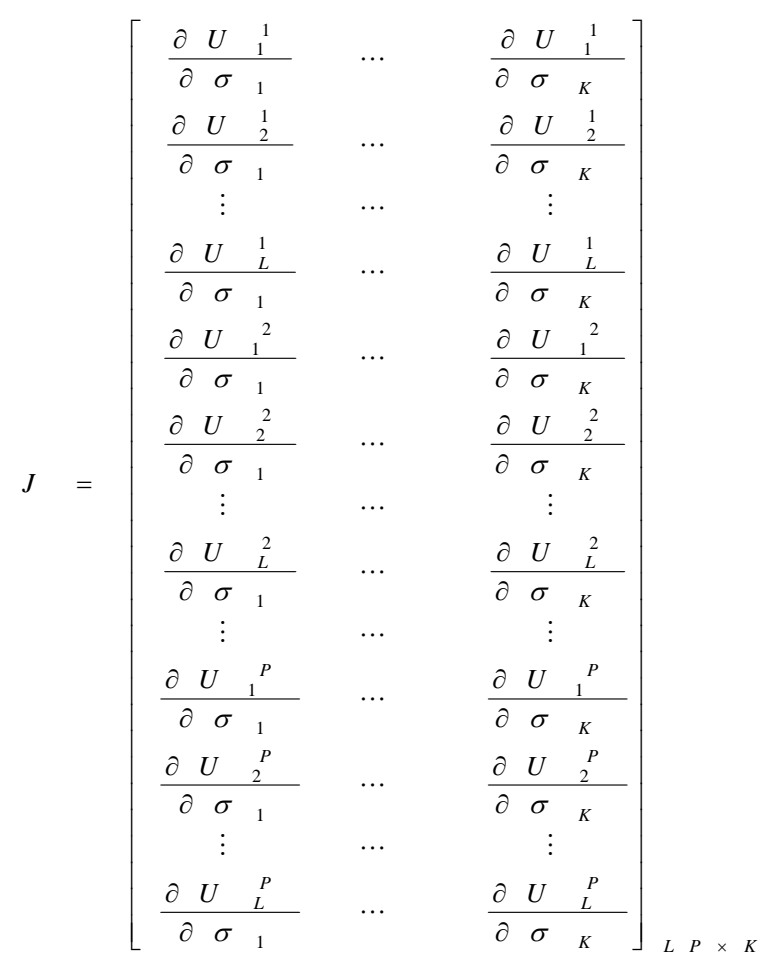

O Jacobiano descreve a mudanças nos voltagens medidos devido a pequenas mudanças de condutividade nos subdomínios de $\Omega$. Daqui em diante vamos denotar o Jacobiano como:

$$
J_{l d, k}=\partial U_{l}^{d} / \partial \sigma_{k},
$$

Uma forma de calcular o Jacobiano é perturbando cada elemento de condutividade e calcula-se a mudança em cada eletrodo, fazemos isso para cada padrão de corrente $d=1,2,3, \ldots, P$ e para todos os $k=1,2, \ldots, K$ subdomínios de $\Omega$. Então, seja o sistema linear que resulta ao aplicar um método numérico para resolver o problema direto:

$$
A b_{p}=f_{c}
$$

A $k$-ésima coluna do Jacobiano pode ser obtida como:

$$
\frac{\partial b_{p}}{\partial \sigma_{k}}=\frac{\partial\left(A^{-1} f_{c}\right)}{\partial \sigma_{k}},
$$

então o lado direito de (2.45) pode ser expandida da seguinte maneira: 


$$
\frac{\partial\left(A^{-1} f_{c}\right)}{\partial \sigma_{k}}=-A^{-1} \frac{\partial A}{\partial \sigma_{k}} A^{-1} f_{c}=-A^{-1} \frac{\partial A}{\partial \sigma_{k}} b_{p}
$$

Se na solução numérica do problema direito se usa o método dos Elementos Finitos então a derivada $\partial A / \partial \sigma_{k}$ pode ser calculado como:

$$
\frac{\partial A(m, i)}{\partial \sigma_{k}}=-\frac{1}{\sigma_{k}^{2}} \int_{\Delta_{k}} \nabla \varphi_{m} \cdot \nabla \varphi_{i}
$$

onde $\hat{u}=\sum_{k=1}^{K} \alpha_{k} \varphi_{k}$ é a solução aproximada e $\varphi_{k}$ são funções base [57].

Outra implementação que usa o método iterativo de Newton é o chamado método de reconstrução de um passo ou algoritmo NOSER [22], [23]. Neste método temos $L$ eletrodos alocados em $\partial \Omega$. Para assegurar uma boa resolução espacial usamos $L-1$ diferentes padrões de correntes $\vec{I}^{d}=\left(I_{1}^{d}, I_{2}^{d}, . ., I_{L}^{d}\right), d=1,2, . . L-1$ e obtemos as correspondentes medidas de voltagens $\vec{V}^{d}=\left(V_{1}^{d}, V_{2}^{d}, \ldots, V_{L}^{d}\right), d=1,2, . . L-1$.

Denotamos como $\vec{U}^{d}=\left(U_{1}^{d}(\sigma), U_{2}^{d}(\sigma), . ., U_{L}^{d}(\sigma)\right), d=1,2, . . L-1$ padrões de voltagens que é produzido ao injetar o $d$-ésimo padrão de corrente para uma distribuição de condutividade $\sigma$.

O problema inverso consiste em determinar a condutividade $\sigma$ que minimiza

$$
\vec{V}^{d}-\vec{U}^{d}(\sigma),
$$

onde $d=1,2, . ., L-1$.

A distribuição de condutividade é dada como:

$$
\sigma(p)=\sum_{k=1}^{K} \sigma_{k} \chi_{k}(p)
$$


onde $\chi_{k}$ é a função característica no $k$-ésimo elemento, e nesse elemento a condutividade é tida como constante.

O problema inverso encontra o mínimo de $\sigma_{k}, k=1,2, . ., K$. Uma maneira de fazer isso é pelo método dos mínimos quadrados. Assim, temos que minimizar o erro:

$$
E_{r}(\sigma)=\sum_{d=1}^{L-1}\left\|\vec{V}^{d}-\vec{U}^{d}(\sigma)\right\|^{2}
$$

e um mínimo pode ser achado fazendo:

$$
0=\frac{\partial E_{r}(\sigma)}{\partial \sigma_{k}}, k=1,2, . ., K
$$

Dada uma condutividade inicial $\sigma_{k}$, o método iterativo de Newton pode ser utilizado para achar uma nova solução.

Outra abordagem para resolver o problema inverso é o chamado método de sensibilidade. Este método é baseado no teorema da de sensibilidade o qual foi derivado por Geselowitz e Lehr [31] e depois foi linearizado por Murai e Kagawa [39].

Sejam $\psi, \phi$ potenciais elétricos em resposta aos padrões de corrente $I_{\psi}, I_{\phi}$ injetados através dos pares de eletrodos $\left(A_{\wp}-B_{\wp}\right.$ e $\left.C_{\wp}-D_{\wp}\right)$ respectivamente como se mostra na figura (2.1). Então a mudança de impedância mútua $\delta Z$ do sistema de quatro eletrodos é dado como:

$$
\delta Z=\frac{\delta \phi_{\mathrm{A}_{\wp} B_{\wp}}}{I_{\phi}}=\frac{\delta \phi_{C_{\S} D_{\wp}}}{I_{\psi}}=-\int_{\Omega} \delta \sigma \frac{\nabla \phi^{*}}{I_{\theta}} \cdot \frac{\nabla \psi}{I_{\psi}} d \Omega,
$$

onde $\phi^{*}=\phi+\delta \phi$ é a mudança do potencial que é causada pela mudança na condutividade $\sigma^{*}=\sigma+\delta \sigma$. Assim:

$$
\delta Z=-\int_{\Omega} \delta \sigma \frac{\nabla \phi}{I_{\theta}} \cdot \frac{\nabla \psi}{I_{\psi}} d \Omega+O\left((\delta \sigma)^{2}\right) \approx-\int_{\Omega} \delta \sigma \frac{\nabla \phi}{I_{\theta}} \cdot \frac{\nabla \psi}{I_{\psi}} d \Omega,
$$

onde $\delta \sigma \ll \sigma$. 


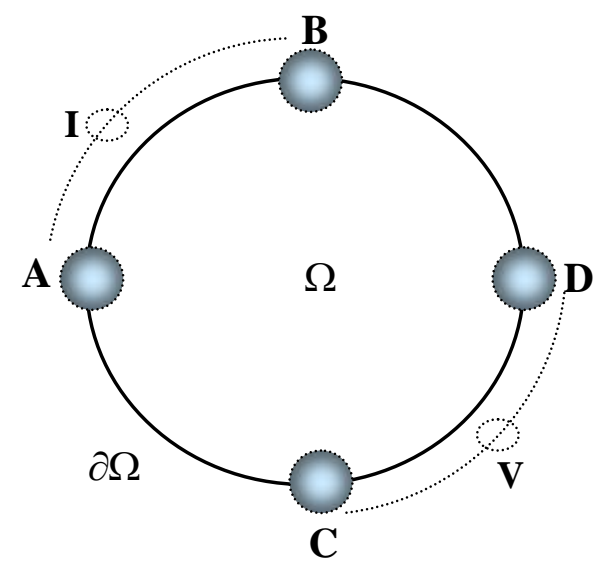

Figura 2.1 A corrente é injetada no interior de $\Omega$ através dos eletrodos A-B e mede-se a voltagem V através dos eletrodos C-D.

A impedância mútua no sistema de quatro eletrodos [61] é dada como:

$$
Z=\frac{\phi_{A_{\wp} B_{\wp}}}{I_{\phi}}=\frac{\phi_{C_{\wp} D_{\wp}}}{I_{\psi}}=-\int_{\Omega} \sigma \frac{\nabla \phi}{I_{\theta}} \cdot \frac{\nabla \psi}{I_{\psi}} d \Omega .
$$

A forma discreta de (2.50) e (2.51) ficam da seguinte maneira:

$$
\begin{gathered}
\delta Z=\sum_{k=1}^{K} \delta \sigma S_{d l, k}, \\
Z=\sum_{k=1}^{K} \sigma_{k} S_{d l, k},
\end{gathered}
$$

onde:

$$
S_{d l, k}=-\int_{\Omega_{k}} \frac{\nabla u^{d}}{I_{d}} \cdot \frac{\nabla u^{l}}{I_{l}} d \Omega_{k},
$$

sendo $u^{d}, u^{l}$ distribuições de voltagens quando o $d$-ésimo e o $l$-ésimo padrão de correntes é usado. Os coeficientes $S_{d l, k}$ formam a chamada matriz de sensibilidade.

Talvez, o método mais difundido que usa a matriz de sensibilidade para resolver o problema inverso seja o método backprojection [3],[42]. 
Em [61] podemos encontrar uma solução inversa que usa a matriz de sensibilidade baseada no método dos gradientes conjugados (GC) cujo algoritmo é dado da seguinte maneira:

(1) Calcular o vetor de voltagem de referência e a matriz de sensibilidade para uma distribuição inicial $\hat{\sigma}_{0}$ :

$$
u\left(\hat{\sigma}^{(0)}\right), S\left(\hat{\sigma}^{(0)}\right)
$$

(2) Mensurar os padrões de voltagens e produzir um vetor de mudanças relativas.

$$
\eta_{d}=\frac{V_{d}^{\prime}\left(\sigma^{\prime}\right)}{V_{d}(\sigma)}, d=1,2, . ., P
$$

(3) Definir os parâmetros de controle: erro mínimo de convergência $\varepsilon_{s}$, número máximo de passos inversos $\delta_{s}$ e o número máximo de iterações $\delta_{c}$ do método dos gradientes conjugados.

(4) Fazer a primeira estimação de um vetor de erro e do vetor de condutividade.

$$
\left\{\begin{array}{l}
e^{(1)}=\left[e_{1}^{(1)}, \ldots, e_{P}^{(1)}\right]^{T}, e_{d}^{(1)}=\eta_{d}-\sum_{d=1}^{P} \frac{\eta_{d}}{P} \\
\hat{\sigma}^{(1)}=\left[\hat{\sigma}_{1}^{(1)}, \ldots, \hat{\sigma}_{K}^{(1)}\right]^{T}, \hat{\sigma}_{k}^{(1)}=\frac{\sigma_{0}}{\sum_{d=1}^{P} \frac{\eta_{d}}{P}}
\end{array}\right.
$$

(5) Normalizar a matriz de sensibilidade: $\bar{S}=\left[\bar{S}_{d l, k}\right]$,

$$
\bar{s}_{d l, k}^{(n)}=\frac{s_{d l, k}\left(\hat{\sigma}_{k}^{(n)}\right)}{\sum_{k=1}^{K} s_{d l, k}\left(\hat{\sigma}_{k}^{(0)}\right)}
$$

(6) Resolver o problema inverso usando o método GC e atualizar as mudanças relativas no vetor de condutividade.

$$
\gamma_{r}=-\bar{S}^{-1} \cdot e
$$

(7) Atualizar o vetor de condutividade. 


$$
\hat{\sigma}^{(n+1)}=\hat{\sigma}^{(n)}\left(1+\gamma_{r}^{(n+1)}\right)
$$

(8) Resolver o problema direto e atualizar o vetor de voltagens e a matriz de sensibilidade.

$$
u\left(\hat{\sigma}^{(n+1)}\right), \quad S\left(\hat{\sigma}^{(n+1)}\right)
$$

(9) Atualizar o vetor de erros.

$$
e_{d}^{(n+1)}=\eta_{d}-\frac{u_{d}^{\prime}\left(\hat{\sigma}_{k}^{(n+1)}\right)}{u_{d}\left(\hat{\sigma}_{k}^{(0)}\right)}
$$

(10) Verificar se os parâmetros de controle têm sido atingidos:

$$
\|e\| \leq \varepsilon_{s} \text {, ou iterações } \geq \delta_{s} \text { ? }
$$

(11) Se não se conseguiu a convergência ou o máximo numero de iterações tem sido alcançado então retornar ao passo (5) ate que os parâmetros de controle sejam alcançados.

Nos últimos anos, métodos probabilísticos (algoritmos genéticos [46], simulated annealing, enxame de partículas, ant-colony, etc ) vem sendo aplicados para resolver o problema inverso. A grande motivação é que esses métodos não usam a informação do gradiente dos potenciais e se espera que uma implementação satisfatória obtenha boas soluções numéricas e melhore o tempo computacional.

O problema de minimização dos métodos probabilísticos é:

$$
\begin{gathered}
\min _{\sigma} \frac{1}{2}\left\|\vec{V}^{d}-\vec{U}^{d}(\sigma)\right\|^{2}, \\
0 \leq \sigma_{k} \leq 1
\end{gathered}
$$

e algumas outras restrições que dependem das especificações do método probabilístico utilizado [11], [46]. 


\section{ESTUDOS NUMÉRICOS USANDO O EIDORS}

O EIDORS (Electrical Impedance and Diffuse Optical Tomography Reconstruction Software [1], [60]) é um software aberto voltado ao uso de reconstrução de imagens em TIE e tomografia ótica difusa (DOT). Este software facilita a pesquisa no que se refere à reconstrução de imagens pois seus algoritmos podem ser modificáveis ou mesmo usados para comparação com novos desenvolvimentos. Os códigos foram implementados em MATLAB e, na sua concepção inicial, o software resolve o problema direto e inverso num domínio bidimensional. Atualmente apareceu a versão para problemas tridimensionais EIDORS 3D (http://eidors3d.sourceforge.net). O problema direto é resolvido usando o método numérico dos elementos finitos e para resolver o problema inverso se usam técnicas de regularização.

\subsection{Características da malha}

Consideramos o domínio como sendo o disco unitário e construímos duas malhas triangulares para resolver o problema direto e o problema inverso. Como é preciso uma solução acurada do problema direto a malha deve ser mais fina comparado à malha que se usa para resolver o problema inverso. Em ambos os casos o pólo $r=0$ faz parte da malha.

Usamos uma malha triangular para resolver o problema inverso (Figura 3.1 (a)) com as seguintes características:

Número de nós $=353$.

Número de elementos $=672$.

Número de eletrodos alocados na fronteira (os elementos correspondentes à locação de eletrodos fica colorida) $=16$.

A malha para resolver o problema direto (Figura 3.1 (b)) tem as seguintes características:

Número de nós $=1377$.

Número de elementos $=2688$. 


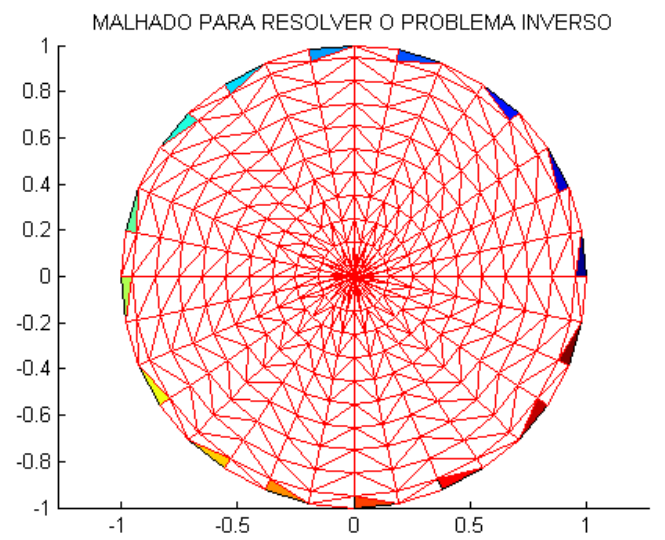

(a)

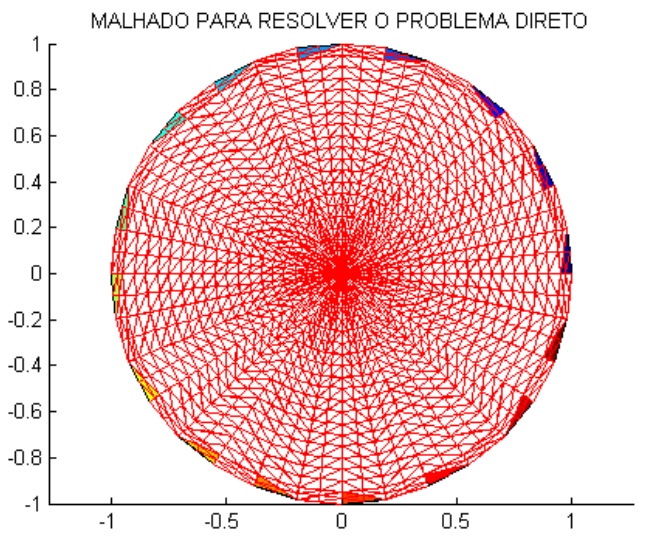

(b)

Figura 3.1 (a) Malha para resolver o problema inverso, (b) Malha para resolver o problema direto.

O EIDORS teve como uma de suas motivações a reconstrução de resistividade dentro de um volume condutor $\rho$. Porém, a resistividade é a inversa da condutividade $\sigma$. Assim a equação (2.13) fica:

$$
\nabla \cdot \frac{1}{\rho} \nabla u=0
$$

Como primeiro exemplo a condutividade é dividida numa região não homogênea com valor (200) e no restante da região tem o valor (400) conforme é mostrada na figura 3.2. 


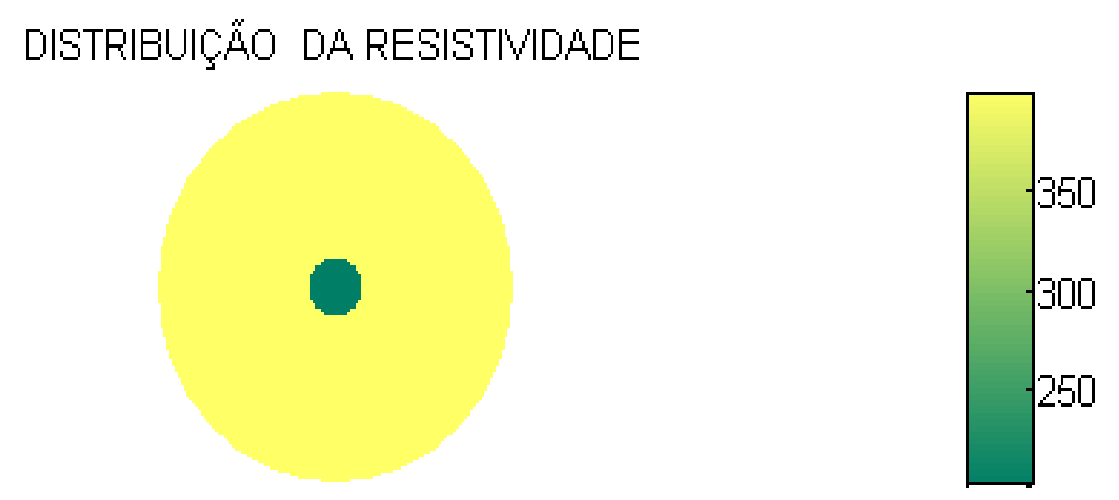

Figura 3.2 Distribuição de resistividade.

\subsection{Tratamento do Problema Direto}

Para resolver o problema direto consideramos a equação (2.13) junto às condições de fronteira dadas com o modelo completo de eletrodos (2.22 - 2.27).

Seja $u_{h}$ a solução generalizada que resolve o problema direto, $\phi_{k}$ funções bases lineares e constantes por partes com suporte limitado tal que:

$$
\phi_{k}(x, y)=\left\{\begin{array}{l}
1, \text { no vértice } i \\
0, \text { em outro lugar }
\end{array},\right.
$$

então:

$$
u_{h}=\sum_{k=1}^{K} \alpha_{k} \phi_{k}
$$

onde $U_{i}$ é o valor do potencial no nó $i$ a ser determinado e $K$ o número total de nós.

A distribuição discreta de condutividade fica:

$$
\sigma=\sum_{k=1}^{K} \sigma_{k} \psi_{k},
$$

onde $\psi_{k}$ são funções bases constante por partes. 
As voltagens $U^{h}$ nos eletrodos são aproximadas como:

$$
U^{h}=\sum_{d=1}^{L-1} \beta_{d} \eta_{d}
$$

onde $\eta_{1}=[1,-1,0, \ldots, 0], \eta_{2}=[1,0,-1,0, . ., 0], \ldots, \eta_{L-1}=[1,0, \ldots, 0,-1]$ e $\eta_{d} \in \mathfrak{R}^{L} . \quad$ Com isto, asseguramos que $\sum_{l=1}^{L} U_{l}=0$, onde $L$ é o numero de eletrodos alocados na fronteira e $\beta_{d}$ são coeficientes a ser determinados.

Como mostrado em [51] para $(v, V), v \in H^{1}(\Omega), V \in \mathfrak{R}^{L}$ :

$$
B_{s}((u, U),(v, V))=\sum_{l=1}^{L} I_{l} V_{l},
$$

onde:

$$
B_{s}((u, U),(v, V))=\int_{\Omega} \sigma \nabla u \cdot \nabla v d x+\sum_{l=1}^{L} \frac{1}{z_{l}} \int_{e_{l}}\left(u-U_{l}\right)\left(v-V_{l}\right) d S .
$$

O sistema linear fica:

$$
A b_{p}=f_{c}
$$

onde $b_{p}=(\alpha, \beta)^{T} \in \mathfrak{R}^{K+L-1}, \quad A \in \mathfrak{R}^{(K+L-1) \times(K+L-1)}$ é uma matriz simétrica que tem a forma:

$$
A=\left(\begin{array}{cc}
B & C \\
C^{T} & D_{b}
\end{array}\right)
$$

e no lado direito de ( 3.7 ) se encontram os padrões de corrente:

$$
f_{c}=\left(\begin{array}{l}
0 \\
\sum_{l=1}^{L} I_{l}\left(\eta_{k}\right)_{l}
\end{array}\right)=\left(\begin{array}{l}
\tilde{0} \\
\tilde{I}
\end{array}\right),
$$


onde $\tilde{0}=(0, \ldots, 0)^{T} \in \mathfrak{R}^{K}, \tilde{I}=\left(I_{1}-I_{2}, I_{1}-I_{3}, \ldots, I_{1}-I_{L}\right)^{T} \in \mathfrak{R}^{L-1}$.

Quando consideramos as funções bases $\phi_{k}$ e os vetores $\eta_{k}$ na forma variacional (3.6) os blocos da matriz A ficam:

$$
\begin{aligned}
B(i, j) & =\int_{\Omega} \sigma \nabla \phi_{i} \cdot \nabla \phi_{j} d x+\sum_{l=1}^{L} \frac{1}{z_{l}} \int_{e_{l}} \phi_{i} \phi_{j} d S, i, j=1,2 . ., K \\
C(i, j) & =-\left(\frac{1}{z_{1}} \int_{e_{l}} \phi_{i} d S-\frac{1}{z_{j+1}} \int_{e_{j+1}} \phi_{i} d S\right), i=1,2 ., K, j=1,2, . ., L-1 \\
D_{b}(i, j) & =\sum_{l=1}^{L} \frac{1}{z_{l}} \int_{e_{l}}\left(\eta_{i}\right)_{l}\left(\eta_{j}\right)_{l} d S \\
& = \begin{cases}\frac{\left|e_{1}\right|}{z_{1}}, & i \neq j \\
\frac{\left|e_{1}\right|}{z_{1}}+\frac{\left|e_{j+1}\right|}{z_{j+1}}, i=j, \quad i, j=1,2, . ., L-1 .\end{cases}
\end{aligned} .
$$

O sistema linear (3.7) é resolvido usando fatorização de Choleski, pois a formulação pelo método dos elementos finitos leva à matriz $A$ a ser simétrica e definida positiva.

Inicialmente o problema direto é aplicado para fazer as simulações das medições das voltagens nos eletrodos.

Dados de entrada:

Número de eletrodos $=16$.

Padrões de corrente trigonométrica $=I_{16 \times 15}$ (15 padrões de corrente e 16 eletrodos). Impedância de contato eletrodo-volume condutor $=z_{16 \times 1}$

O problema direto fornece a distribuição do potencial nos 1377 nós para os 15 padrões de corrente injetados $U_{1377 \times 15}$, sendo que 16 voltagens para cada um dos 15 padrões $V_{16 \times 15}$ correspondem às voltagens simuladas nos eletrodos. 


\subsection{Tratamento do Problema Inverso}

A distribuição de condutividade inicial é escolhida de maneira que as voltagens na fronteira de $\Omega$ são decompostas como:

$$
U_{l}(\sigma) \approx \frac{\sigma}{\sigma_{r}} U_{l}\left(\sigma_{r}\right)
$$

onde $\sigma_{r}$ é uma condutividade constante arbitrária. Então resulta que a melhor condutividade homogênea $\sigma_{0}$ é a que minimiza o seguinte funcional:

$$
\left\|V-\frac{\sigma_{0}}{\sigma_{r}} U\left(\sigma_{r}\right)\right\|,
$$

onde a solução é obtida pelo método dos mínimos quadrados.

Assim, se em nosso algoritmo escolhermos $\sigma_{r}=1.0$, resolvemos o problema direto para esta condutividade homogênea obtendo $U_{16 \times 15}\left(\sigma_{r}=1.0\right)$ medidas de voltagens nos eletrodos e o valor da condutividade (homogênea) inicial $\sigma_{0}$.

Novamente resolvemos o problema direto com a condutividade inicial e calculamos o Jacobiano $J_{\sigma_{0}}$. Para resolver o problema de minimização (2.40) é preciso definir a matriz de regularização $R$ e o parâmetro de regularização $\alpha$ com o propósito de utilizar o método de Gauss-Newton com regularização de Tikhonov para aproximar a distribuição de resistividade.

O parâmetro de regularização e a matriz de regularização usam informações a priori do problema. Na solução deste problema o parâmetro de regularização foi calculado aplicando o método da curva - L [17] (como mostra a figura 3.3) e a matriz de regularização é construída tentando obter imagens com certa suavidade. 


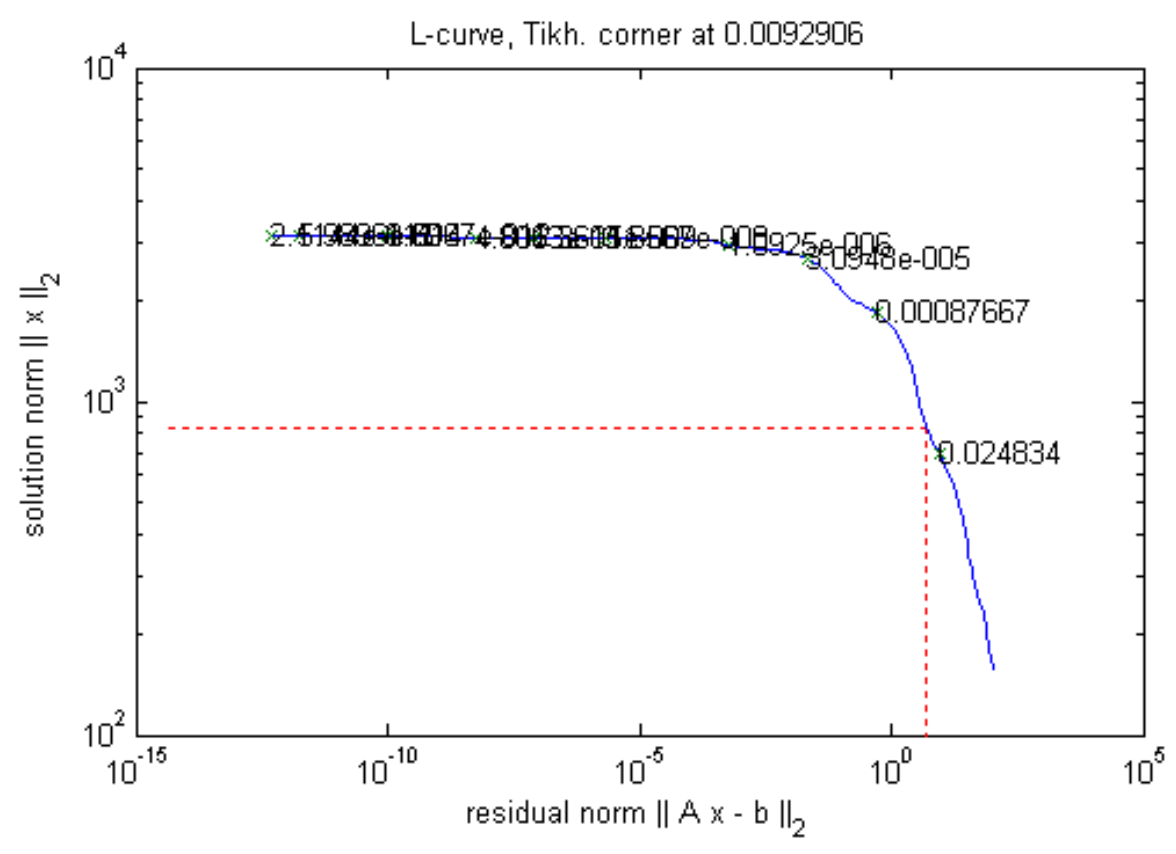

Figura 3.3 Gráfico da curva $L$.

A solução do problema inverso usa um processo iterativo do tipo Gauss-Newton com regularização de Tikhonov, assim:

$$
\begin{aligned}
& \text { para } \mathrm{i}=1 \text { : max it } \\
& \rho_{\mathrm{i}}=\rho_{i-1}+\delta \rho_{i-1} \\
& \delta \rho_{i-1}=\left(J_{i-1}{ }^{T} J_{i-1}+\alpha R^{T} R\right)^{-1}\left[J_{i-1}{ }^{T}\left(V-U_{\sigma_{i-1}}\right)-\alpha R^{T} R \rho_{i-1}\right]
\end{aligned}
$$

onde $\max i t=5$

Assim, obtemos a seguinte imagem da reconstrução da resistividade:

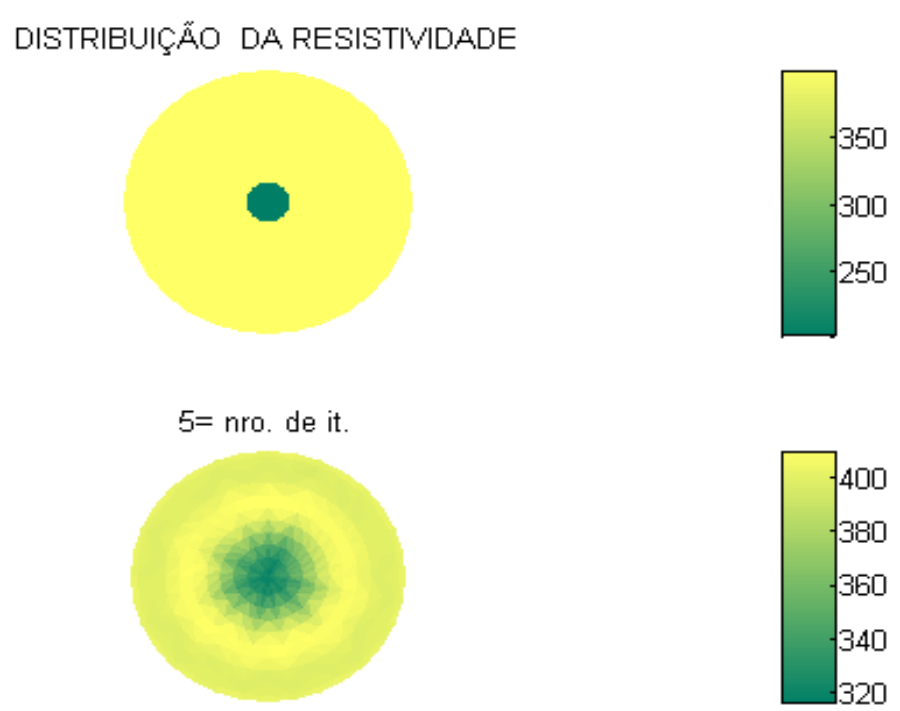

Figura 3.4 A distribuição de resistividade e a aproximação após a $5^{\mathrm{a}}$ iteração. 
O erro é medido da seguinte maneira:

$$
E_{i}=\left\|V-U_{\sigma_{i}}\right\|
$$

onde $i$ é o número de iterações. Assim, após a quinta iteração temos que $E_{5}=1.544 \times 10^{-6}$ sendo que o tempo computacional gasto pelo processo iterativo foi de aproximadamente 23.2s ( $\mathrm{s}$ = segundos) e as malhas para resolver o problema direto e inverso foram geradas antes do processo iterativo e gastaram aproximadamente 35s para serem geradas.

Na figura 3.4 podemos observar que o método iterativo usado consegue identificar a região onde fica a interface de valores de resistividade e este processo fica mais preciso na medida em que as iterações aumentam. 


\section{ESTUDOS NUMÉRICOS DO PROBLEMA DIRETO}

A reconstrução da condutividade no interior de um volume é essencialmente baseada na capacidade de calcular o campo elétrico no interior do mesmo quando os padrões de corrente são injetados na fronteira. Esta é a característica principal do modelo direto. Portanto, o objetivo do problema direto é estimar a distribuição do potencial no interior e na fronteira de um volume para, seqüencialmente, gerar observações na mesma.

O problema direto pode ser resolvido, em alguns casos, analiticamente [21] derivando equações para avaliar o potencial elétrico em algum ponto do volume ou pode ser resolvido numericamente.

Sabendo que nas aplicações da TIE a distribuição de condutividade ou resistividade podem ser funções continuas ou descontínuas por partes, a continuação resolvemos o problema de difusão numericamente para diferentes tipos de distribuição e testamos os métodos em problemas onde a solução analítica é conhecida.

\subsection{O problema de difusão com coeficientes contínuos em coordenadas polares}

A equação a ser considerada é mais geral que a dada em (2.13), a saber, por considerar uma função fonte definida em $\Omega$.

A equação de difusão em coordenadas polares e em dimensão 2 é :

$$
\frac{-1}{r}\left[\frac{\partial}{\partial r}\left(\sigma(r, \theta) r \frac{\partial u}{\partial r}\right)+\frac{\partial}{\partial \theta}\left(\frac{\sigma(r, \theta)}{r} \frac{\partial u}{\partial \theta}\right)\right]=f(r, \theta), \quad \text { em } \Omega
$$


onde vamos considerar $\Omega$ como sendo o disco unitário $\Omega=\{(r, \theta) / 0<r<1,0 \leq \theta<2 \pi\}$ e os coeficientes $\sigma(r, \theta)$ não homogêneos porém contínuos. A equação (4.1) é munida de uma das seguintes condições de fronteira de tipo Dirichlet ou Neumann:

$$
\left\{\begin{array}{l}
u(1, \theta)=g(\theta), \quad \text { em } \partial \Omega \\
\text { ou } \\
\sigma \frac{\partial u}{\partial n}=h(\theta), \quad \text { em } \partial \Omega
\end{array},\right.
$$

onde $n$ é a direção normal e $f, g$ ou $h$ são funções conhecidas.

\section{Discretização dos eixos radial e angular.}

\section{Eixo radial}

Para evitar um tratamento especial na singularidade do pólo usamos a seguinte malha modificada:

No caso quando a condição de fronteira é Dirichlet temos:

$r_{i}=(i-1 / 2) h_{r}$, onde $h_{r}=\frac{2}{2 M+1}, i=1, \ldots, M+1$. Assim, $r_{i}-r_{i-1}=h_{r}, i=2, \ldots, M+1 \mathrm{e}$ $r_{i \pm 1 / 2}=r_{i} \pm \frac{h_{r}}{2}, r_{M+1}=1.0$. O pólo ocorre em $r_{1 / 2}=0$ e não faz parte da malha como mostrado na figura (4.1).

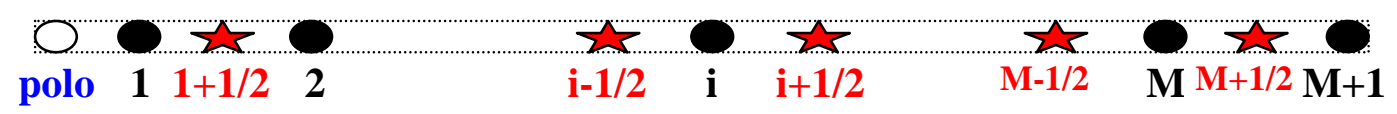

Figura 4.1 Subdivisão do eixo radial considerando a condição de Dirichlet.

No caso quando em que a condição de fronteira é de tipo Neumann temos: $r_{i}=(i-1 / 2) h_{r}$, onde $h_{r}=\frac{1}{M}, i=1, \ldots, M$. Assim, $r_{i}-r_{i-1}=h_{r}, i=2, \ldots, M$ e $r_{i \pm 1 / 2}=r_{i} \pm \frac{h_{r}}{2}$, $r_{M+1 / 2}=1.0$.

A figura 4.2 mostra esta discretização.

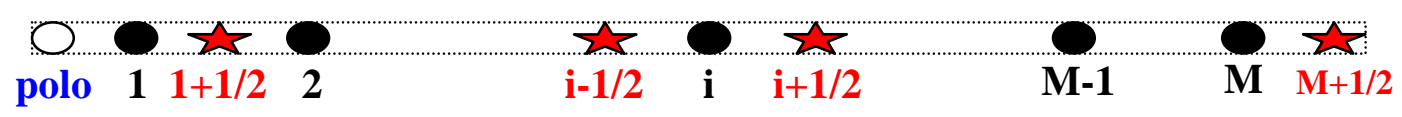

Figura 4.2 Subdivisão do eixo radial considerando a condição de Neumann. 


\section{Eixo angular}

$\theta_{j}=j h_{\theta}, \quad h_{\theta}=\frac{2 \pi}{N}, j=1,2, \ldots, N, \theta_{j \pm 1 / 2}=\theta_{j} \pm \frac{h_{\theta}}{2} \mathrm{e}$

$\theta_{j}-\theta_{j-1}=h_{\theta}, j=2, . ., N$.

A figura 4.3 mostra a discretização no eixo angular.

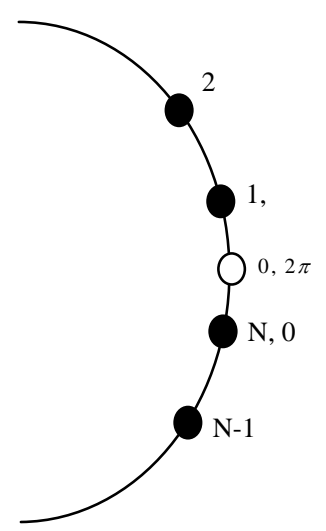

Figura 4.3 Subdivisão do eixo angular.

Considerando as seguintes notações: $u_{i, j} \approx u\left(r_{i}, \theta_{j}\right), f_{i, j} \approx f\left(r_{i}, \theta_{j}\right)$,

$\sigma_{i \pm 1 / 2, j}=\sigma\left(r_{i} \pm 0.5 h_{r}, \theta_{j}\right), \sigma_{i, j \pm 1 / 2}=\sigma\left(r_{i}, \theta_{j} \pm 0.5 h_{\theta}\right)$ e $g_{j}=g\left(\theta_{j}\right)$. Para discretizar a equação

(4.1) se usou o método de diferenças finitas centrais com um stêncil de cinco pontos [36].

Assim, temos:

$$
-\frac{1}{r_{i}}\left[\begin{array}{l}
\left(r_{i+1 / 2} \sigma_{i+1 / 2, j} \frac{u_{i+1, j}-u_{i, j}}{h_{r}}-r_{i-1 / 2} \sigma_{i-1 / 2, j} \frac{u_{i, j}-u_{i-1, j}}{h_{r}}\right) / h_{r}+ \\
\left(\frac{\sigma_{i, j+1 / 2 j}}{r_{i}} \frac{u_{i, j+1}-u_{i, j}}{h_{\theta}}-\frac{\sigma_{i, j-1 / 2 j}}{r_{i}} \frac{u_{i, j}-u_{i, j-1}}{h_{\theta}}\right) / h_{\theta}
\end{array}\right]=f_{i, j},
$$

onde $1 \leq i \leq M, 1 \leq j \leq N$. 
As condições de periodicidade na variável $\theta$ são dadas da seguinte maneira:

$$
\left\{\begin{array}{l}
u_{i, 0}=u_{i, M} \\
u_{i, M+1}=u_{i, 1}
\end{array},\right.
$$

onde $u_{i, 0}$ e $u_{i, M+1}$ são substituídos em (4.3).

Quando consideramos condições de fronteira tipo Dirichlet, temos que $u_{N+1 j}=g_{j}$ e no caso de considerar à condição de Neumann temos $\left.\sigma(1, \theta) \frac{\partial u}{\partial r}\right|_{r=1}=h(\theta)$.

Na condição de fronteira tipo Neumann em $i=M$, consideramos a própria equação de difusão. Assim temos:

$$
-\frac{r_{M-1 / 2} \sigma_{M-1 / 2, j}}{h_{r}^{2}} u_{M-1, j}-\frac{\sigma_{M, j-1 / 2}}{r_{M} h_{\theta}^{2}} u_{M, j-1}+\left[\frac{r_{M-1 / 2} \sigma_{M-1 / 2, j}}{h_{r}^{2}}+\left(\frac{\sigma_{M, j+1 / 2}+\sigma_{M, j-1 / 2}}{r_{M} h_{\theta}^{2}}\right)\right] u_{M, j}-\frac{\sigma_{M, j+1 / 2}}{r_{M} h_{\theta}^{2}} u_{M, j+1}=r_{M} f_{M, j}+\frac{h_{j}}{h_{r}}
$$

O sistema linear que resulta desta discretização é da forma: $A x=b$, onde $A=\left[a_{i j}\right]$ é uma matriz de dimensão $M N$, no caso onde as condições de fronteira são de tipo Dirichlet temos que $A$ é simétrica e definida positiva e no caso de considerar a condição de fronteira tipo Neumann temos que $A$ e uma matriz simétrica e podemos mostrar [25] que é semi definida positiva.

\section{Resultados Numéricos}

Dadas as seguintes notações:

$$
\left\{\begin{array}{l}
u_{e x}=\text { solução exata } \\
u_{a p}=\text { solução aproximada } \\
e_{\max }=\text { erro relativo na norma do maximo } \\
e_{L 2}=\text { erro relativo na norma } \mathrm{L}^{2}
\end{array}\right.
$$

Os erros usados para testar nossos algoritmos são: 
erro relativo na norma do máximo:

$$
e_{\max }=\left\|u_{e x}-u_{a p}\right\|_{\infty} /\left\|u_{e x}\right\|_{\infty}
$$

e erro relativo na norma $\mathrm{L}^{2}$ :

$$
e_{L 2}=\left\|u_{e x}-u_{a p}\right\|_{L^{2}} /\left\|u_{e x}\right\|_{\infty}
$$

A taxa de convergência é calculada pela fórmula:

$$
t x_{c}=\log _{2}\left(\frac{e_{M / 2}}{e_{M}}\right)
$$

onde: $e_{M}$ denota o erro na norma do máximo relativo ou L2 relativo, considerando $M$ subdivisões do eixo radial. As subdivisões no intervalo angular é $N=2 M$.

Para obter a solução aproximada $u_{a p}$ usando o método de diferenças finitas descrito acima, resolvemos o sistema linear $A\left(u_{a p}\right)=b$ usando métodos diretos e métodos iterativos.

Considerando métodos diretos usamos fatorização de Choleski e para testar os algoritmos usamos soluções conhecidas do problema (4.1) dadas em [36]:

\section{Problema (1)}

$$
\left\{\begin{array}{l}
u(r, \theta)=\exp (r(\cos (\theta)+\operatorname{sen}(\theta))), \\
\sigma(r, \theta)=r^{2} \operatorname{sen}^{2}(\theta)+r \cos (\theta)+1.1
\end{array}\right.
$$




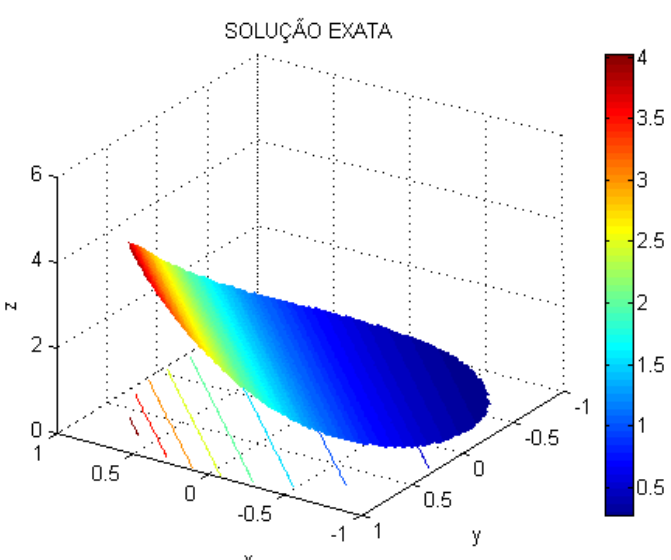

(a)

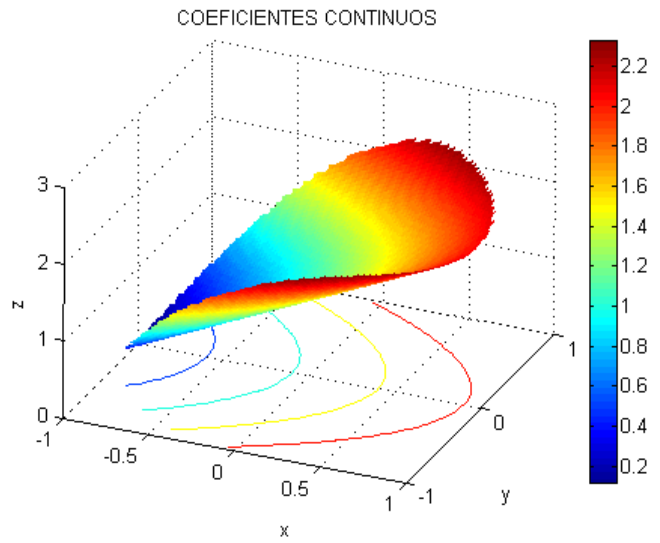

(b)

Figura 4.4 (a) Gráfico da solução exata, (b) Gráfico de $\sigma(r, \theta)$.

As figuras 4.4, (a) e (b) foram geradas considerando coordenadas cartesianas:

$$
\left\{\begin{array}{l}
x=r \cos (\theta) \\
y=r \operatorname{sen}(\theta)
\end{array},\right.
$$

e mostram a distribuição das variáveis $u(r, \theta)$ e dos coeficientes contínuos $\sigma(r, \theta)$ respectivamente no disco unitário.

\section{Resultados:}

Tabela 4.1 Aproximação do Problema (1) com condição de fronteira tipo Dirichlet e usando fatorização de Choleski.

\begin{tabular}{|c|c|c|}
\hline \multicolumn{3}{|c|}{ Fatorização de Choleski } \\
\hline $\mathbf{M}$ & $E_{L 2}$ & $E_{\max }$ \\
\hline 16 & $6.1936491 \times 10^{-4}$ & 0.0011683 \\
\hline 32 & $1.5007779 \times 10^{-4}$ & $2.8271419 \times 10^{-4}$ \\
\hline 64 & $3.69344449 \times 10^{-5}$ & $6.9544314 \times 10^{-5}$ \\
\hline 128 & $9.1611034 \times 10^{-6}$ & $1.7255221 \times 10^{-5}$ \\
\hline
\end{tabular}

A taxa de convergência fica:

Tabela 4.2 Taxa de convergência do Problema (1), considerando o erro relativo na norma L2.

\begin{tabular}{|c|c|}
\hline $\mathbf{M}$ & $t x_{c}$ \\
\hline 16 & - \\
\hline 32 & 2.04 \\
\hline 64 & 2.02 \\
\hline 128 & 2.01 \\
\hline
\end{tabular}


Das tabelas 4.1 e 4.2 podemos observar a convergência quadrática o qual é uma conseqüência da discretização de segunda ordem usada.

Também podemos usar métodos iterativos para resolver o sistema linear. Dado que a matriz do sistema linear é simétrica usamos o método dos gradientes conjugados com précondicionamento Choleski incompleto (PGC) e o método PGC com drop tolerance. Os detalhes da implementação deste último método são dados em [47]. O sistema linear foi resolvido usando as rotinas correspondentes a estes métodos do MATLAB versão 7, obtendose os seguintes resultados:

Tabela 4.3 Aproximação do Problema (1) com condição de fronteira tipo Dirichlet e usando PGC com pré-condicionamento Choleski Incompleto.

\begin{tabular}{|c|c|c|c|c|}
\hline \multicolumn{5}{|c|}{ PGC - Choleski Incompleto } \\
\hline $\mathbf{M}$ & $E_{L 2}$ & $E_{\max }$ & Iterações & Tempo (s) \\
\hline 16 & $6.1913716 \times 10^{-4}$ & 0.0011674 & 24 & 0.15625 \\
\hline 32 & $1.4976962 \times 10^{-4}$ & $2.8114731 \times 10^{-4}$ & 44 & 0.5 \\
\hline 64 & $3.8360657 \times 10^{-5}$ & $6.5745694 \times 10^{-5}$ & 79 & 3.421875 \\
\hline 128 & $1.0499728 \times 10^{-5}$ & $1.9168523 \times 10^{-5}$ & 154 & 35.65625 \\
\hline
\end{tabular}

Tabela 4.4 Aproximação do Problema (1) com condição de fronteira tipo Dirichlet usando PGC com pré-condicionamento Choleski Incompleto com drop tolerance.

\begin{tabular}{|c|c|c|c|c|}
\hline \multicolumn{5}{|c|}{ PGC - Choleski Incompleto com drop tolerance $=10^{-3}$} \\
\hline $\mathbf{M}$ & $E_{L 2}$ & $E_{\max }$ & Iterações & Tempo (s) \\
\hline 16 & $6.1953557 \times 10^{-4}$ & 0.0011685 & 6 & 0.15675 \\
\hline 32 & $1.5032993 \times 10^{-4}$ & $2.8203244 \times 10^{-4}$ & 8 & 0.40625 \\
\hline 64 & $3.7186787 \times 10^{-5}$ & $6.9194729 \times 10^{-5}$ & 14 & 2.28125 \\
\hline 128 & $1.0314064 \times 10^{-5}$ & $2.3044259 \times 10^{-5}$ & 27 & 19.765625 \\
\hline
\end{tabular}

Tabela 4.5 Taxa de convergência ao aplicar PGC com pré-condicionamento considerando o erro relativo na norma $L 2$.

\begin{tabular}{|c|c|}
\hline $\mathbf{M}$ & $t x_{c}$ \\
\hline 16 & - \\
\hline 32 & 2.04 \\
\hline 64 & 2.01 \\
\hline 128 & 1.9 \\
\hline
\end{tabular}

O critério de parada usado nos métodos iterativos é: 


$$
\left\|b-A x_{(i)}\right\| /\|b\|<10^{-6}
$$

Nas tabelas 4.3 e 4.4 se observa que quando usamos o método de Choleski incompleto com drop tolerance obtemos um ganho de tempo computacional na medida em que reduzimos o tamanho do passo e na tabela 4.5 podemos observar a ordem de convergência quadrática.

\section{Problema (2)}

$$
\left\{\begin{array}{l}
u(r, \theta)=\operatorname{sen}(r \cos (\theta)) \operatorname{sen}(r \operatorname{sen}(\theta)), \\
\sigma(r, \theta)=\exp (r(\cos (\theta)+\operatorname{sen}(\theta)))
\end{array}\right.
$$

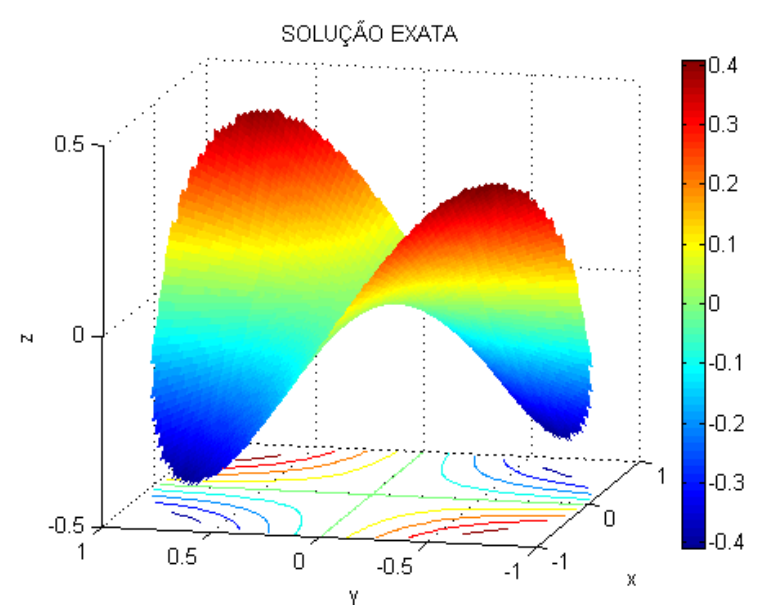

(a)

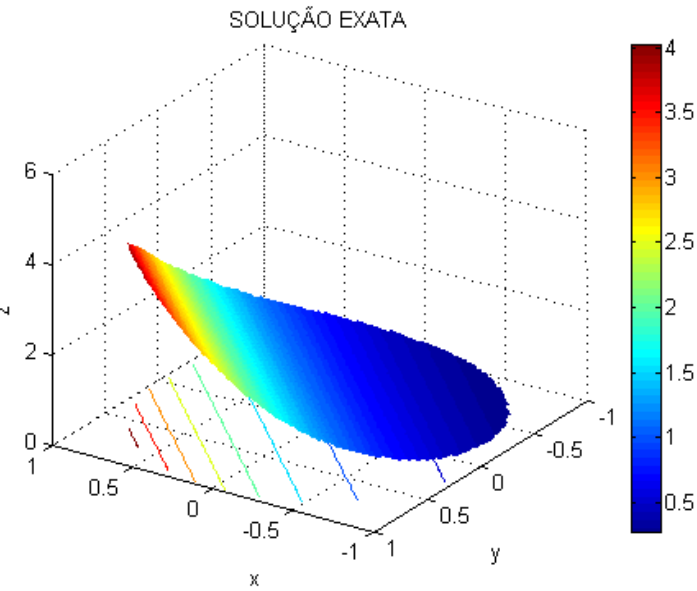

(b)

Figura 4.5 (a) Gráfico da solução exata, (b) Gráfico de $\sigma(r, \theta)$.

\section{Resultados:}

Tabela 4.6 Aproximação do Problema (2) com condição de fronteira tipo Dirichlet e usando fatorização de Choleski.

\begin{tabular}{|c|c|c|}
\hline \multicolumn{3}{|c|}{ Fatorização de Choleski } \\
\hline $\mathbf{M}$ & $E_{L 2}$ & $E_{\max }$ \\
\hline 16 & 0.0027051 & 0.0029793 \\
\hline 32 & $6.4079641 \times 10^{-4}$ & $7.0663607 \times 10^{-4}$ \\
\hline 64 & $1.5606043 \times 10^{-4}$ & $1.7211521 \times 10^{-4}$ \\
\hline 128 & $3.8489361 \times 10^{-5}$ & $4.2482815 \times 10^{-5}$ \\
\hline
\end{tabular}


Tabela 4.7 Taxa de convergência do Problema (2) respeito do erro relativo na norma L2.

\begin{tabular}{|c|c|}
\hline $\mathbf{M}$ & $t x_{c}$ \\
\hline 16 & - \\
\hline 32 & 2.08 \\
\hline 64 & 2.03 \\
\hline 128 & 2.01 \\
\hline
\end{tabular}

A tabela 4.7 mostra que a taxa de convergência é compatível com a discretização de segunda ordem usada.

Usando os métodos iterativos gradientes conjugados com pré-condicionamento Choleski incompleto (PGC) e o método PGC com drop tolerance, temos os seguintes resultados:

Tabela 4.8 Aproximação do Problema (2) com condição de fronteira tipo Dirichlet e usando PGC com pré-condicionamento Choleski Incompleto.

\begin{tabular}{|c|c|c|c|c|}
\hline \multicolumn{5}{|c|}{ PGC - Choleski Incompleto } \\
\hline $\mathbf{M}$ & $E_{L 2}$ & $E_{\max }$ & Iterações & Tempo (s) \\
\hline 16 & 0.0027056 & 0.0029792 & 23 & 0.15625 \\
\hline 32 & $6.4181419 \times 10^{-4}$ & $7.0698817 \times 10^{-4}$ & 40 & 0.5 \\
\hline 64 & $1.5714453 \times 10^{-4}$ & $1.7274502 \times 10^{-4}$ & 77 & 3.5 \\
\hline 128 & $3.8980834 \times 10^{-5}$ & $4.8992245 \times 10^{-5}$ & 151 & 35.6875 \\
\hline
\end{tabular}

Tabela 4.9 Aproximação do Problema (2) com condição de fronteira tipo Dirichlet e usando PGC com pré-condicionamento Choleski Incompleto com drop tolerance.

\begin{tabular}{|c|c|c|c|c|}
\hline \multicolumn{5}{|c|}{ PGC - Choleski Incompleto com drop tolerance $=10^{-3}$} \\
\hline $\mathbf{M}$ & $E_{L 2}$ & $E_{\max }$ & Iterações & Tempo (s) \\
\hline 16 & 0.0027052 & 0.0029794 & 6 & 0.15625 \\
\hline 32 & $6.4246953 \times 10^{-4}$ & $7.0870142 \times 10^{-4}$ & 8 & 0.40625 \\
\hline 64 & $1.5567015 \times 10^{-4}$ & $1.7241548 \times 10^{-4}$ & 13 & 2.28125 \\
\hline 128 & $3.3645628 \times 10^{-5}$ & $4.6711596 \times 10^{-5}$ & 25 & 19.921875 \\
\hline
\end{tabular}

Tabela 4.10 Taxa de convergência ao aplicar PGC com pré-condicionamento.

\begin{tabular}{|c|c|}
\hline $\mathbf{M}$ & $t x_{c}$ \\
\hline 16 & - \\
\hline 32 & 2.07 \\
\hline 64 & 2.04 \\
\hline 128 & 2.1 \\
\hline
\end{tabular}


O critério de parada usado nos métodos iterativos do problema (2) é o dado em (4.7) onde novamente podemos observar das tabelas 4.8 e 4.9 o ganho de tempo computacional na medida em que reduzimos o tamanho do passo.

Considerando a condição de fronteira tipo Neumann, temos os seguintes resultados para os problemas (1) e (2).

Tabela 4.11 Aproximação do Problema (1) com condição de fronteira tipo Neumann e usando PGC com pré-condicionamento Choleski Incompleto com drop tolerance.

\begin{tabular}{|c|c|c|c|c|}
\hline \multicolumn{5}{|c|}{ Problema (1) } \\
\hline $\mathbf{M}$ & $E_{L 2}$ & $E_{\max }$ & Iterações & Tempo (s) \\
\hline 16 & 0.0033189 & 0.0055591 & 6 & 0.171875 \\
\hline 32 & $7.9769944 \times 10^{-4}$ & 0.0013271 & 10 & 0.40625 \\
\hline 64 & $1.9574212 \times 10^{-4}$ & $3.2537913 \times 10^{-4}$ & 18 & 2.390625 \\
\hline 128 & $4.8495202 \times 10^{-5}$ & $8.0481329 \times 10^{-5}$ & 35 & 20.234375 \\
\hline
\end{tabular}

Tabela 4.12 Taxa de convergência do problema (1) com condição de fronteira tipo Neumann.

\begin{tabular}{|c|c|}
\hline $\mathbf{M}$ & $t x_{c}$ \\
\hline 16 & - \\
\hline 32 & 2.05 \\
\hline 64 & 2.02 \\
\hline 128 & 2.01 \\
\hline
\end{tabular}

Tabela 4.13 Aproximação do Problema (2) com condição de fronteira tipo Neumann usando PGC com pré-condicionamento Choleski Incompleto com drop tolerance.

\begin{tabular}{|c|c|c|c|c|}
\hline \multicolumn{5}{|c|}{ Problema (2) } \\
\hline $\mathbf{M}$ & $E_{L 2}$ & $E_{\max }$ & Iterações & Tempo (s) \\
\hline 16 & 0.0069783 & 0.0079139 & 6 & 0.171875 \\
\hline 32 & 0.0016918 & 0.0019159 & 10 & 0.4375 \\
\hline 64 & $4.1698847 \times 10^{-4}$ & $4.7223551 \times 10^{-4}$ & 18 & 2.375 \\
\hline 128 & $1.0347735 \times 10^{-4}$ & $1.1743769 \times 10^{-4}$ & 30 & 20.09375 \\
\hline
\end{tabular}

Tabela 4.14 Taxa de convergência do problema (2) com condição de fronteira tipo Neumann.

\begin{tabular}{|c|c|}
\hline $\mathbf{M}$ & $t x_{c}$ \\
\hline 16 & - \\
\hline 32 & 2.04 \\
\hline 64 & 2.01 \\
\hline 128 & 2.01 \\
\hline
\end{tabular}


Na solução numérica da equação (4.1) com condição de fronteira tipo Neumann é preciso fazer uma normalização, neste caso foi feito uma normalização pela fronteira para termos uma solução única, pois as soluções deste problema diferem em uma constante. No apêndice A temos uma descrição de alguns tipos de normalização.

O critério de parada usado para gerar as tabelas 4.11 e 4.13 é dado em (4.7) e as taxas de convergência das tabelas 4.12 e 4.14 mostram serem compatíveis com a ordem de discretização usada.

\subsection{O problema de difusão com coeficientes descontínuos}

Na maioria de aplicações da TIE os coeficientes $\sigma$ são descontínuos em $\Omega$, sendo que existem interfaces $\Gamma \subset \Omega$ que separam as regiões onde os coeficientes têm valores característicos. Assim se precisa de métodos apropriados para lidar com as descontinuidades dos coeficientes.

Sabendo que na maioria de aplicações da técnica de TIE em dimensão 2 o domínio é um quadrado, um disco ou uma deformação destes volumes, resolvemos o problema de difusão pelo método dos volumes finitos modificados (MVFM) [14],[20], com o objetivo de termos uma técnica que proporcione resultados acurados.

O problema de difusão pode ser escrito da seguinte maneira:

$$
-\operatorname{div}(\sigma(x) \operatorname{grad}(u(x)))=f(x), x \in \Omega,
$$

onde div é o operador divergência e grad é o operador gradiente.

A equação (4.9) é sujeita as condições de fronteira tipo Dirichlet ou Neumann e $\sigma(x)$ é descontinua numa interface $\Gamma \subset \Omega$. As interfaces que são consideradas usando este método são paralelas aos eixos coordenados. 
Devido à natureza dos processos, geralmente, as componentes do fluxo através da interface $\Gamma$ definidas como $-\sigma \operatorname{grad}(u) . v$, onde $v$ é o vetor normal unitário são suaves apesar de que os coeficientes $\sigma$ e as derivadas da solução são descontínuos.

Para colocar as idéias principais deste método começamos nossa discussão do método de MVFM com a solução do problema de difusão em coordenadas retangulares e em dimensão 1.

\subsubsection{Discretização por volumes finitos modificados em problemas de dimensão 1} Problemas com condição de fronteira tipo Dirichlet:

Em dimensão 1 a equação de difusão é:

$$
\left\{\begin{array}{l}
W^{\prime}(x)=f(x), \quad W(x)=-\sigma(x) u^{\prime}(x), \quad 0<x<1 . \\
u(0)=a_{1}, \quad u(1)=a_{2}
\end{array}\right.
$$

onde: $\sigma(x)$ é um coeficiente de difusão conhecida, $W(x)$ é o fluxo e $f(x)$ é o termo fonte.

O problema vem acompanhado de condições de continuidade da solução $u$ e do fluxo $W$ numa interface $x=\xi \in \Omega$ :

$$
[u]=[W]=0, \quad x=\xi,
$$

onde: $[\varphi]$ denota a diferença dos limites à direita e a esquerda de $\varphi$ no ponto de descontinuidade $\xi$.

Consideramos como domínio do problema o intervalo $\Omega=[0,1]$ e as principais condições para a existência de uma solução contínua neste problema são:

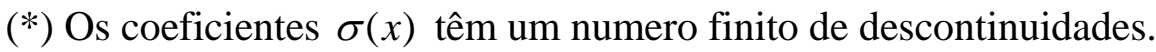

(*) O lado direito $f(x) \in C^{2}[0,1]$. 


\section{Malha}

Usaremos a seguinte malha:

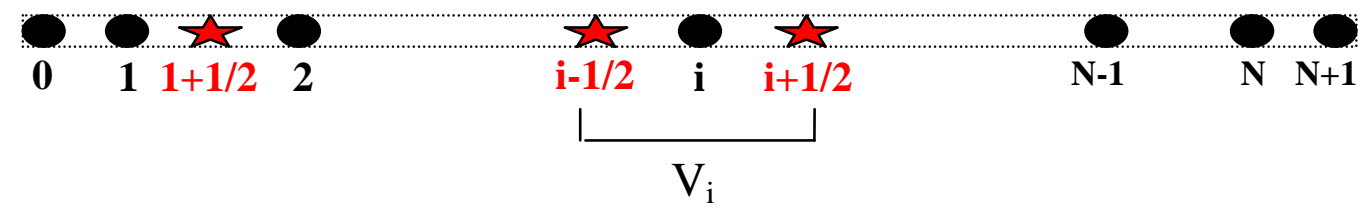

Figura 4.6 Subdivisão do intervalo [0,1].

Os pontos nodais são: $x_{0}=0, \quad x_{1}=h / 2, \quad x_{i}=x_{i-1}+h, i=2, \ldots, N, \quad x_{N+1}=1$, onde $h=1 / N$.

Os volumes finitos são definidos como: $V_{i}=\left(x_{i-1 / 2}, x_{i+1 / 2}\right)$, onde $x_{i \pm 1 / 2}=x_{i} \pm \frac{h}{2}$.

\section{Método dos volumes finitos modificados}

Integrando a equação dada em (4.1) num volume finito, temos:

$$
W_{i+1 / 2}-W_{i-1 / 2}=\int_{x_{i-1 / 2}}^{x_{i+1 / 2}} f(x) d x, \quad i=1,2, \ldots, N
$$

Seja:

$$
-u^{\prime}(x)=\frac{W(x)}{\sigma(x)}
$$

Integrando essa equação no intervalo $\left(x_{i}, x_{i+1}\right)$ :

$$
-\left(u_{i+1}-u_{i}\right)=-\int_{x_{i}}^{x_{i+1}} u^{\prime}(x) d x=\int_{x_{i}}^{x_{i+1}} \frac{W(x)}{\sigma(x)} d x
$$

Considerando que $W(x)$ é duas vezes continuamente diferenciável na interface podemos expandir ao redor de $x_{i+1 / 2}$ na série de Taylor:

$$
W(x)=W_{i+1 / 2}+\left(x-x_{i+1 / 2}\right) W^{\prime}\left(x_{i+1 / 2}\right)+\frac{\left(x-x_{i+1 / 2}\right)^{2}}{2} W^{\prime \prime}(\eta), \quad \eta \in\left(x_{i}, x_{i+1}\right)
$$


Substituindo a primeira derivada do fluxo em $x_{i+1 / 2}$ pelo esquema backward de diferenças finitas chegamos a seguinte expressão:

$$
-\left(u_{i+1}-u_{i}\right)=W_{i+1 / 2} \int_{x_{i}}^{x_{i+1}} \frac{d x}{\sigma(x)}+\frac{W_{i+1 / 2}-W_{i-1 / 2}}{h} \int_{x_{i}}^{x_{i+1}} \frac{\left(x-x_{i+1 / 2}\right)}{\sigma(x)} d x+O\left(h^{3}\right)
$$

Assim a equação fica:

$$
-\kappa_{i+1 / 2}^{H} \frac{u_{i+1}-u_{i}}{h}=W_{i+1 / 2}+a_{i+1 / 2}\left(W_{i+1 / 2}-W_{i-1 / 2}\right)+\varphi_{i},
$$

onde $\varphi_{i}=O\left(h^{2}\right)$ e:

$$
\begin{gathered}
\kappa_{i+1 / 2}^{H}=\left(\frac{1}{h} \int_{x_{i}}^{x_{i+1}} \frac{d x}{\sigma(x)}\right)^{-1}, \\
a_{i+1 / 2}=\kappa_{i+1 / 2}^{H} \frac{1}{h^{2}} \int_{x_{i}}^{x_{i+1}} \frac{x-x_{i+1 / 2}}{\sigma(x)} d x,
\end{gathered}
$$

$\kappa_{i+1 / 2}^{H}$ é a média harmônica dos coeficientes $\sigma(x)$ sobre a célula $\left(x_{i}, x_{i+1}\right)$.

Por causa de usar os termos $a_{i+1 / 2}, a_{i-1 / 2}$ chamamos este método de media harmônica modificada (HIA) e observamos que em (4.16) no lado direito as ordens dos termos são: $O(1), O(h)$ e $O\left(h^{2}\right)$ respectivamente sendo que se consideramos somente o primeiro termo do lado direito temos o esquema de Samarski [48], onde se prova que esta discretização é $O(h)$-consistente nos pontos de interface e a convergência é de segunda ordem na norma $H^{1}$. Chamaremos a este esquema de (HA).

Denotamos como $F_{i+1 / 2}$ e $F_{i-1 / 2}$ a aproximação dos fluxos $W_{i+1 / 2}$ e $W_{i-1 / 2}$ respectivamente, e denotamos como $y_{i}$ as aproximações dos valores exatos da solução $u\left(x_{i}\right)$. Assim temos as seguintes relações: 


$$
\left\{\begin{array}{l}
-\kappa_{i+1 / 2}^{H} \frac{y_{i+1}-y_{i}}{h}=F_{i+1 / 2}+a_{i+1 / 2}\left(F_{i+1 / 2}-F_{i-1 / 2}\right) \\
-\kappa_{i-1 / 2}^{H} \frac{y_{i}-y_{i-1}}{h}=F_{i-1 / 2}+a_{i-1 / 2}\left(F_{i+1 / 2}-F_{i-1 / 2}\right) .
\end{array}\right.
$$

Dessas relações chegamos a:

$$
\left(1+a_{i+1 / 2}-a_{i-1 / 2}\right)\left(F_{i+1 / 2}-F_{i-1 / 2}\right)=-\kappa_{i+1 / 2}^{H} \frac{y_{i+1}-y_{i}}{h}+\kappa_{i-1 / 2}^{H} \frac{y_{i}-y_{i-1}}{h},
$$

onde os pontos $x_{0}, x_{N+1}$ estão numa distancia $h / 2$ do nó vizinho.

As equações em $i=1$ e $i=N$ são modificados convenientemente para assegurar a convergência desejada [20].

Assim o problema fica:

$$
L_{h} y_{i}=\mu_{i}, \quad i=1,2, \ldots, N,
$$

onde:

$$
L_{h} y_{i}=\left\{\begin{array}{l}
-\frac{4}{3} \frac{1}{h}\left(\kappa_{3 / 2}^{H} \frac{y_{2}-y_{1}}{h}-\kappa_{1 / 2}^{H} \frac{2 y_{1}}{h}\right), \quad i=1 \\
-\left(1+a_{i+1 / 2}-a_{i-1 / 2}\right)^{-1} \frac{1}{h}\left(\kappa_{i+1 / 2}^{H} \frac{y_{i+1}-y_{i}}{h}-\kappa_{i-1 / 2}^{H} \frac{y_{i}-y_{i-1}}{h}\right), \quad i \neq 1, N \\
-\frac{4}{3} \frac{1}{h}\left(\kappa_{N+1 / 2}^{H} \frac{2 y_{N}}{h}-\kappa_{N-1 / 2}^{H} \frac{y_{N}-y_{N-1}}{h}\right), \quad i=N
\end{array}\right.
$$

Para comparar os resultados que produz o método HIA, usaremos o esquema HA e o esquema da media aritmética (AA), onde temos que neste último esquema $a_{i+1 / 2}=a_{i-1 / 2}=0$ e $\kappa_{i+1 / 2}^{H}=0.5\left(\sigma_{i}+\sigma_{i+1}\right)$.

O novo esquema HIA aproxima os fluxos nas laterais dos volumes finitos com exatidão de segunda ordem, independentemente da posição da descontinuidade dos coeficientes $\sigma(x)$ [14]. O preço que pagamos é a necessidade de avaliar $\left(a_{i+1 / 2}-a_{i-1 / 2}\right)$ e $\kappa_{i+1 / 2}^{H}$ com um erro maior o igual que $O\left(h^{2}\right)$. 


\section{Discretização na interface}

Suponha que $\sigma(x)$ é descontínua em $x=\xi, \xi=x_{i}+v h$ para algum $i$ e $0 \leq v \leq 1$. Obviamente que quando $v=0$ e $v=1$ temos as interfaces alinhadas com os pontos nodais.

No caso $0<v<1$ suponha que a descontinuidade fica no intervalo $\left(x_{i}, x_{i+1}\right)$ então $\kappa_{i+1 / 2}^{H}$ é calculada integrando em $\left(x_{i}, \xi\right)$ e $\left(\xi, x_{i+1}\right)$.

Assim no caso em que $\sigma(x)$ é constante por partes temos as seguintes aproximações:

$$
\begin{gathered}
\kappa_{i+1 / 2}^{H}=\left(\frac{v}{\sigma_{i}}+\frac{(1-v)}{\sigma_{i+1}}\right)^{-1}, \\
a_{i+1 / 2}=\frac{1}{2} \frac{v(1-v)\left(\sigma_{i}-\sigma_{i+1}\right)}{(1-v) \sigma_{i}+v \sigma_{i+1}},
\end{gathered}
$$

e conseqüentemente com a seguinte aproximação no fluxo:

$$
F_{i+1 / 2}=\frac{-\kappa_{i+1 / 2}^{H} \frac{y_{i+1}-y_{i}}{h}\left(1-a_{i-1 / 2}\right)-\kappa_{i-1 / 2}^{H} \frac{y_{i}-y_{i-1}}{h}\left(a_{i+1 / 2}\right)}{\left(1+a_{i+1 / 2}\right)\left(1-a_{i-1 / 2}\right)+a_{i+1 / 2} a_{i-1 / 2}}=W_{i+1 / 2}+O\left(h^{2}\right)
$$

atingimos convergência de segunda ordem.

\section{Resultados Numéricos}

Consideramos a descontinuidade em $\xi=1 / 2$.

Quando utilizamos os métodos AA (média Aritmética), HA (média harmônica) a matriz do sistema linear é simétrica definida positiva e o método que usamos para resolver este sistema linear é o método PGC. Porém, quando utilizamos o método HIA (média harmônica modificada) a matriz do sistema linear é não simétrica e para resolver este sistema aplicamos o método dos Bi-gradientes conjugados com pré - condicionamento LU incompleto. 
Para calcular o erro consideramos as normas definidas em (4.5) e (4.6).

O problema usado para testar os algoritmos é:

\section{Problema (3)}

$$
\left\{\begin{array}{l}
u(x)=(1 / \sigma) \sin \left(\frac{\pi x}{2}\right)(x-1 / 2)\left(1+x^{2}\right), \\
\sigma(x)= \begin{cases}1, & 0<x<0.5 \\
10^{-4}, & 0.5<x<1\end{cases}
\end{array}\right.
$$

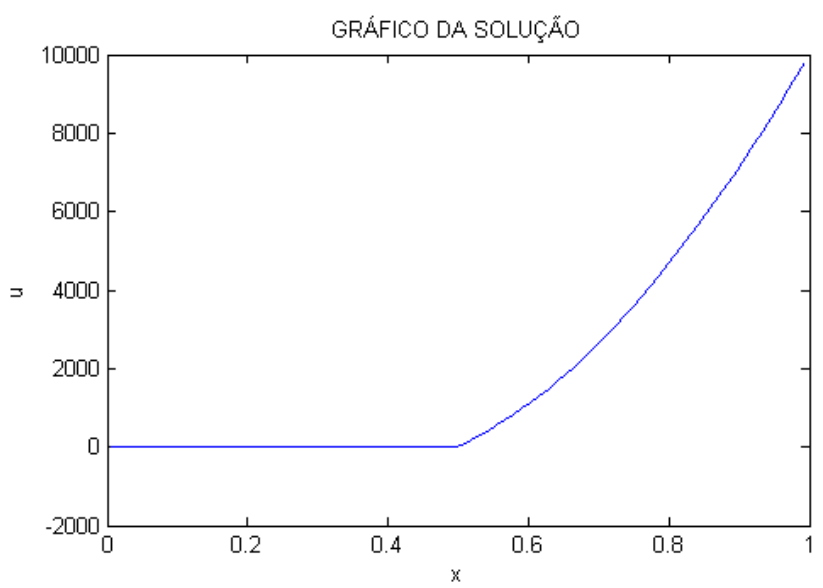

Figura 4.7 Gráfico da solução exata do Problema 3.

Os resultados são comparados com á solução exata nas normas do erro máximo relativo e na norma $L^{2}$ relativa, obtendo-se:

Tabela 4.15 Aproximação aplicando os métodos AA, HA e HIA do Problema (3) usando o erro relativo na norma do máximo.

\begin{tabular}{|c|c|c|c|}
\hline $\begin{array}{c}\text { Erro relativo na } \\
\text { norma do máximo }\end{array}$ & \multicolumn{3}{|c|}{$\sigma=\left\{1,10^{-4}\right\}$} \\
\hline M=N & AA & HA & HIA \\
\hline 12 & $3.14 \times 10^{-2}$ & $4.31 \times 10^{-3}$ & $4.68 \times 10^{-4}$ \\
\hline 22 & $1.53 \times 10^{-2}$ & $1.21 \times 10^{-3}$ & $1.82 \times 10^{-4}$ \\
\hline 42 & $7.55 \times 10^{-3}$ & $3.19 \times 10^{-4}$ & $5.59 \times 10^{-5}$ \\
\hline 82 & $3.74 \times 10^{-3}$ & $8.24 \times 10^{-5}$ & $7.64 \times 10^{-6}$ \\
\hline 162 & $1.86 \times 10^{-3}$ & $2.09 \times 10^{-5}$ & $2.84 \times 10^{-6}$ \\
\hline
\end{tabular}




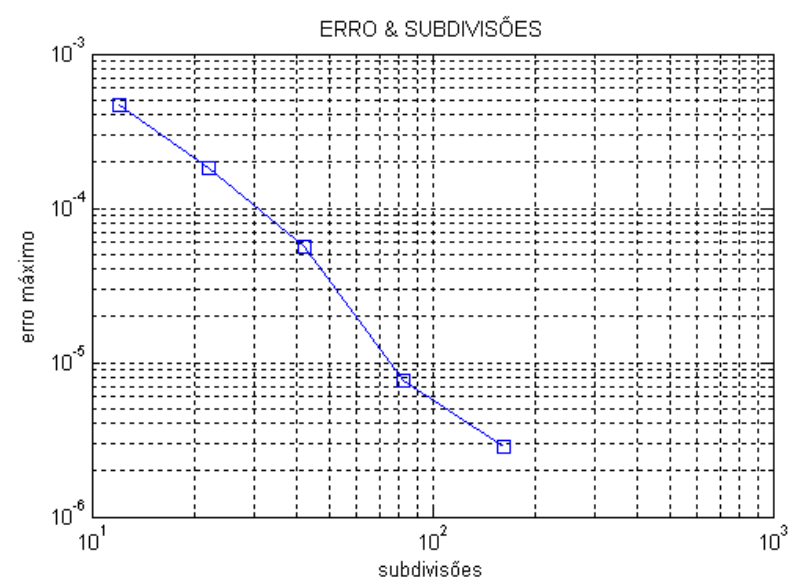

Figura 4.8 Gráfico do erro relativo na norma do máximo no método HIA \& subdivisões no eixo radial no Problema (3).

Tabela 4.16 Aproximação aplicando os métodos AA, HA e HIA do Problema (3) usando o erro relativo na norma $L^{2}$.

\begin{tabular}{|c|c|c|c|}
\hline $\begin{array}{c}\text { Erro relativo na } \\
\text { norma L }\end{array}$ & \multicolumn{3}{|c|}{$\sigma=\left\{1,10^{-4}\right\}$} \\
\hline $\mathbf{M}=\mathbf{N}$ & AA & HA & HIA \\
\hline 12 & $1.47 \times 10^{-2}$ & $2.79 \times 10^{-3}$ & $2.31 \times 10^{-4}$ \\
\hline 22 & $6.79 \times 10^{-3}$ & $7.79 \times 10^{-4}$ & $8.74 \times 10^{-5}$ \\
\hline 42 & $3.23 \times 10^{-3}$ & $2.07 \times 10^{-4}$ & $2.76 \times 10^{-5}$ \\
\hline 82 & $1.57 \times 10^{-3}$ & $5.32 \times 10^{-5}$ & $7.64 \times 10^{-6}$ \\
\hline 162 & $7.71 \times 10^{-4}$ & $1.35 \times 10^{-5}$ & $1.42 \times 10^{-6}$ \\
\hline
\end{tabular}

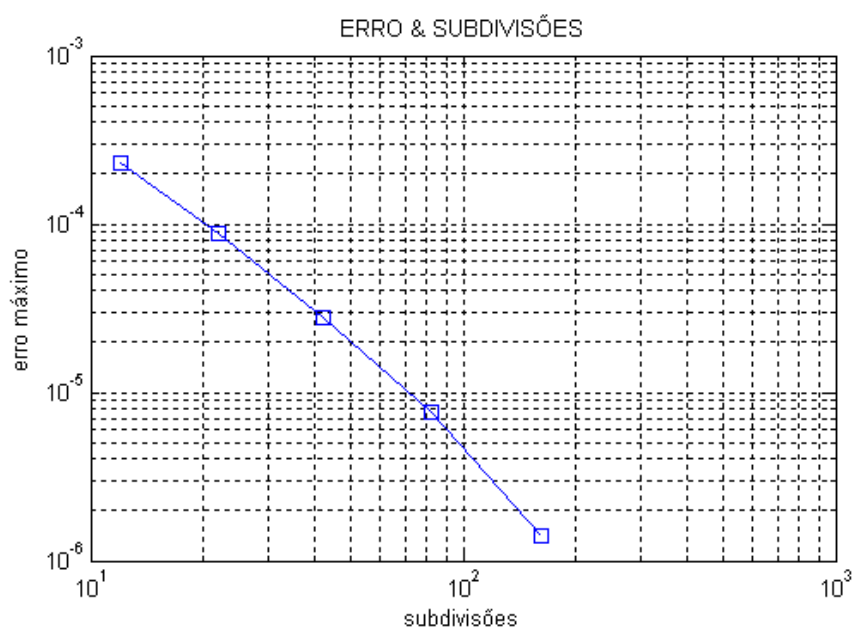

Figura 4.9 Gráfico do erro relativo na norma L2 no método HIA \& subdivisões no eixo radial no Problema (3). 
Destes resultados podemos observar que nas tabelas 4.15 e 4.16 que as ordens de convergência são: para o método AA $O(h)$, para o método HA $O\left(h^{2}\right)$. Também podemos observar dos gráficos 4.8 e 4.9 que o método HIA tem ordem de convergência $O\left(h^{2}\right)$, porém os resultados são mais acurados que o método HA, estes resultados eram os esperados segundo as ordens de discretização usadas.

\subsubsection{Discretização por volumes finitos modificados em problemas de dimensão 2}

Consideramos um domínio bi-dimensional a equação de difusão (4.1) munida das condições de fronteira tipo Dirichlet ou Neumann tem a forma:

$$
\left\{\begin{array}{l}
-\left[\left(\sigma u_{x}\right)_{x}+\left(\sigma u_{y}\right)_{y}\right]=f(x, y), \text { em } \Omega \\
u(x, y)=g(x, y), \text { em } \partial \Omega \\
o u \\
\sigma \frac{\partial u}{\partial n}=h(x, y), \text { em } \partial \Omega
\end{array}\right.
$$

onde consideramos o domínio $\Omega=[0,1] \times[0,1]$.

Considerando as interfaces sendo paralelas às faces dos volumes finitos. $\mathrm{O}$ desenvolvimento em dimensão 2 é o produto tensorial de 2 problemas de dimensão 1 (um para cada direção).

As componentes do fluxo ficam:

$$
W(x, y)=\left[\begin{array}{c}
-\sigma u_{x} \\
-\sigma u_{y}
\end{array}\right]=\left[\begin{array}{l}
W_{1} \\
W_{2}
\end{array}\right]
$$

O gráfico de um volume finito é dado na figura 4.10. 


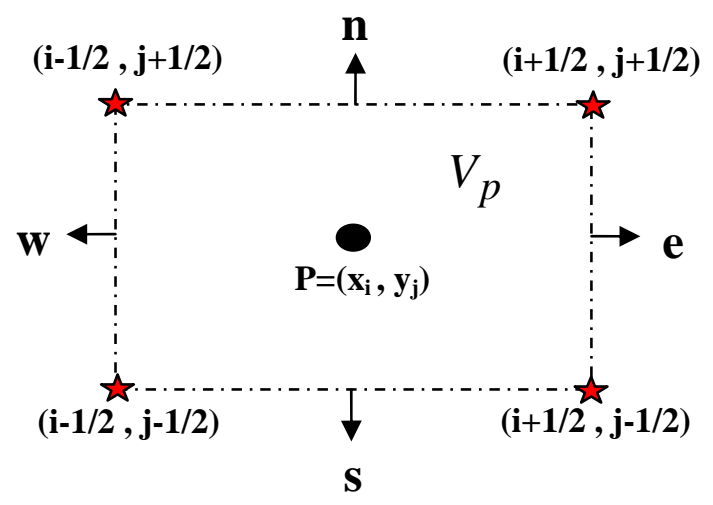

Figura 4.10 Volume finito em coordenadas cartesianas.

Integrando a equação em $V_{p}$ :

$$
\int_{V_{p}} \nabla \cdot W d A=h_{x} h_{y} \varphi_{p}, \quad \varphi_{p}=\frac{1}{h_{x} h_{y}} \int_{V_{p}} f d A
$$

pelo teorema da divergência:

$$
\begin{aligned}
& \int_{V_{p}} \nabla \cdot W d A=\int_{\partial V_{p}} W \cdot n d l \\
& \int_{\partial V_{p}} W \cdot n d l=\int_{x_{i-1 / 2}}^{x_{i+1 / 2}}\left[W_{2}\left(x, y_{j+1 / 2}\right)-W_{2}\left(x, y_{j-1 / 2}\right)\right] d x+\int_{y_{j-1 / 2}}^{y_{j+1 / 2}}\left[W_{1}\left(x_{i+1 / 2}, y\right)-W_{1}\left(x_{i-1 / 2}, y\right)\right] d y
\end{aligned}
$$

Aplicando a regra do ponto médio temos:

$$
h_{y}\left(W_{e}-W_{w}\right)+h_{x}\left(W_{n}-W_{s}\right)=h_{x} h_{y} f_{i j} .
$$

Assim, a discretização fica: 
Na direção $x$ :

$$
\left\{\begin{array}{l}
h_{y}\left[\frac{-4}{3}\left(K_{3 / 2, j}^{H} \frac{y_{2, j-} y_{1, j}}{h_{x}}-K_{1 / 2, j}^{H} \frac{2 y_{1, j}}{h_{x}}\right)\right], i=1, j=1, \ldots, N \\
h_{y}\left[-\left(1+a_{i+1 / 2, j}-a_{i-1 / 2, j}\right)^{-1}\left(K_{i+1 / 2, j}^{H} \frac{y_{i+1, j}-y_{i, j}}{h_{x}}-K_{i-1 / 2, j}^{H} \frac{y_{i, j}-y_{i-1, j}}{h_{x}}\right)\right], i=2, . ., M-1, \quad j=1, \ldots, N \\
h_{y}\left[\frac{-4}{3}\left(K_{N+1 / 2, j}^{H} \frac{2\left(y_{N+1, j} y_{N, j}\right)}{h_{x}}-K_{N-1 / 2, j}^{H} \frac{\left(y_{N, j}-y_{N-1, j}\right)}{h_{x}}\right)\right], i=M, j=1, \ldots, N
\end{array},\right.
$$

e na direção y:

$$
\left\{\begin{array}{l}
h_{x}\left[\frac{-4}{3}\left(K_{i, 3 / 2}^{H} \frac{y_{i, 2-} y_{i, 1}}{h_{y}}-K_{i, 1 / 2}^{H} \frac{2\left(y_{i, 1}-y_{i, 0}\right)}{h_{y}}\right)\right], j=1, i=1, \ldots, M \\
h_{x}\left[-\left(1+a_{i, j+1 / 2}-a_{i, j-1 / 2}\right)^{-1}\left(K_{i, j+1 / 2}^{H} \frac{y_{i, j+1-} y_{i, j}}{h_{y}}-K_{i, j-1 / 2}^{H} \frac{y_{i, j}-y_{i, j-1}}{h_{y}}\right)\right], j=2, \ldots N-1, \quad i=1, \ldots, M \\
h_{x}\left[\frac{-4}{3}\left(K_{i, M+1 / 2}^{H} \frac{2\left(y_{i, M+1-} y_{i, M}\right)}{h_{y}}-K_{i, M-1 / 2}^{H} \frac{\left(y_{i, M}-y_{i, M-1}\right)}{h_{y}}\right)\right], j=N, i=1, \ldots, M
\end{array}\right.
$$

Na figura 4.11 temos o domínio $[0,1] \times[0,1]$ e a interface centrada nos pontos médios do domínio.

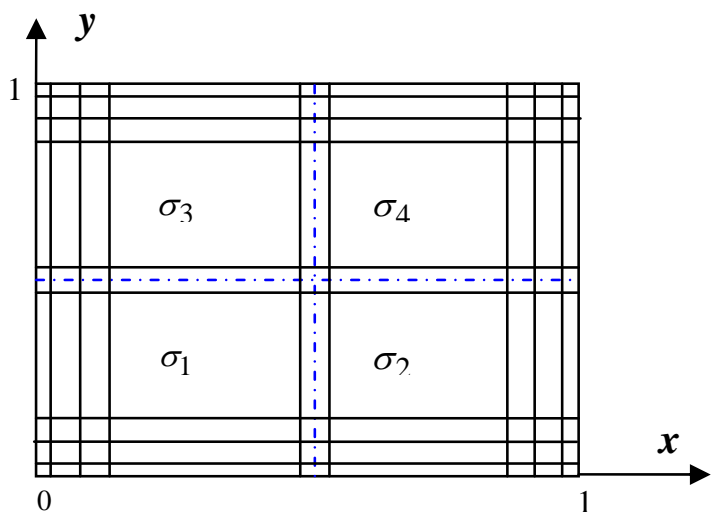

Figura 4.11 O Domínio [0,1]x[0,1] e a localização da interface. 


\section{Resultados Numéricos:}

\section{Problema (4)}

$$
\left\{\begin{array}{l}
u(x, y)=\frac{1}{\sigma} \operatorname{sen}\left(\frac{\pi x}{2}\right)\left(x-\xi_{1}\right)\left(y-\xi_{2}\right)\left(1+x^{2}+y^{2}\right) \\
\sigma(x, y)=\left\{\begin{array}{l}
\sigma_{1}=10^{-2} ; 0 \leq x<\xi_{1}, 0 \leq y<\xi_{2} \\
\sigma_{2}=1 ; \xi_{1}<x \leq 1,0 \leq y<\xi_{2} \\
\sigma_{3}=10^{-4} ; 0 \leq x<\xi_{1}, \xi_{2}<y \leq 1 \\
\sigma_{4}=10^{+5} ; \xi_{1}<x \leq 1, \xi_{2}<y \leq 1
\end{array}\right.
\end{array}\right.
$$

onde $\left(\xi_{1}, \xi_{2}\right)=(0.5,0.5)$.

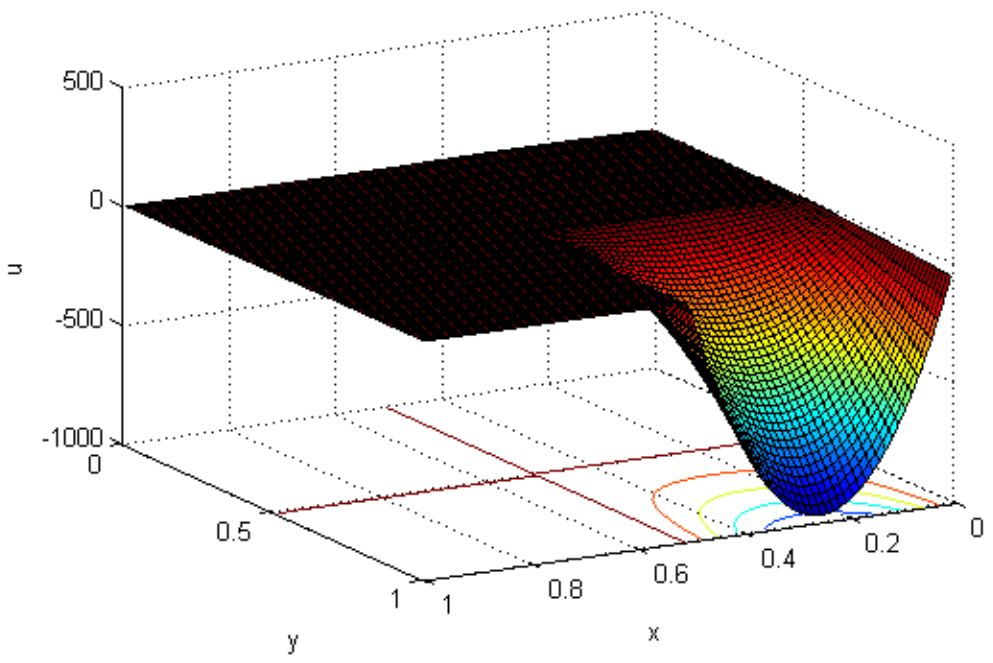

Figura 4.12 Gráfico da solução exata do Problema (4).

Para resolver o sistema linear usamos o método de fatorização de Choleski para ambos os métodos (HA e HIA) e o erro é dado usando a norma do máximo como descrito em (4.5).

Tabela 4.17 Aproximação aplicando os métodos HA e HIA do Problema (4) usando o erro relativo na norma do máximo.

\begin{tabular}{|c|c|c|}
\hline GRID & HA & HIA \\
\hline $\mathbf{N = M}$ & $\begin{array}{c}\text { Erro relativo na norma do } \\
\text { máximo }\end{array}$ & $\begin{array}{c}\text { Erro relativo na norma do } \\
\text { máximo }\end{array}$ \\
\hline 12 & 0.0157335 & $2.562509 \times 10^{-4}$ \\
\hline 22 & 0.005368 & $6.656319 \times 10^{-5}$ \\
\hline 42 & 0.0017031 & $1.825298 \times 10^{-5}$ \\
\hline 82 & $4.894295 \times 10^{-4}$ & $4.819306 \times 10^{-6}$ \\
\hline 162 & $1.335411 \times 10^{-4}$ & $1.242216 \times 10^{-6}$ \\
\hline
\end{tabular}


Podemos observar na tabela 4.17 que com o método HIA obtemos resultados mais acurados e mantemos a ordem de convergência quadrática, porém gastamos maior tempo computacional.

Também resolvemos o sistema linear do método HA usando o método iterativo dos gradientes conjugados com pré-condicionador Choleski Incompleto e o critério de parada e da tolerância relativa, dado em (4.7) $t o l=10^{-6}$, e no esquema HIA usamos o método dos Bigradientes conjugados com pré-condicionador LU Incompleto e tolerância relativa tol $=10^{-6}$. Quando usamos os métodos PGC e Bi-PGC com a fatorização incompleta no modo usual (sem drop-tolerance) temos os seguintes resultados:

Tabela 4.18 Aproximação aplicando os métodos HA e HIA do Problema (4) usando o erro relativo na norma do máximo.

\begin{tabular}{|c|c|c|c|c|c|c|}
\hline GRID & \multicolumn{3}{|c|}{ HA } & \multicolumn{3}{c|}{ HIA } \\
\hline N=M & $\begin{array}{c}\text { Erro relativo na } \\
\text { norma do máximo }\end{array}$ & Iter & Tempo & $\begin{array}{c}\text { Erro relativo na } \\
\text { norma do máximo }\end{array}$ & Iter & Tempo \\
\hline 12 & 0.015838 & 13 & 0.140625 & $2.562112 \times 10^{-4}$ & 14 & 0.234375 \\
\hline 22 & 0.005367 & 23 & 0.203125 & $6.656383 \times 10^{-5}$ & 22 & 0.3125 \\
\hline 42 & 0.001696 & 39 & 0.484375 & $1.825301 \times 10^{-5}$ & 42 & 0.859375 \\
\hline 82 & $4.877827 \times 10^{-4}$ & 78 & 2.84375 & $4.818712 \times 10^{-6}$ & 76 & 4.921875 \\
\hline 162 & $1.332667 \times 10^{-4}$ & 142 & 24.28125 & $1.2416931 \times 10^{-6}$ & 147 & 45.828125 \\
\hline
\end{tabular}

Quando usamos os métodos PCG e Bi-PCG com a fatorização incompleta com droptolerance $=10^{-3}$ temos:

Tabela 4.19 Aproximação aplicando os métodos HA e HIA do Problema (4) usando o erro relativo na norma do máximo com drop tolerance.

\begin{tabular}{|c|c|c|c|c|c|c|}
\hline GRID & \multicolumn{3}{|c|}{ HA } & \multicolumn{3}{|c|}{ HIA } \\
\hline $\mathbf{N}=\mathbf{M}$ & $\begin{array}{l}\text { Erro relativo na } \\
\text { norma do máximo } \\
\text { relativo }\end{array}$ & Iter & Tempo & $\begin{array}{c}\text { Erro relativo na } \\
\text { norma } \\
\text { do máximo relativo }\end{array}$ & Iter & Tempo \\
\hline 12 & 0.015838 & 4 & 0.125 & $2.562531 \times 10^{-4}$ & 3 & 0.21875 \\
\hline 22 & 0.005366 & 5 & 0.171875 & $6.649025 \times 10^{-5}$ & 4 & 0.28125 \\
\hline 42 & 0.001696 & 8 & 0.34375 & $1.825383 \times 10^{-5}$ & 7 & 0.578125 \\
\hline 82 & $4.877825 \times 10^{-4}$ & 13 & 1.65625 & $4.819987 \times 10^{-6}$ & 12 & 2.46875 \\
\hline 162 & $1.332631 \times 10^{-4}$ & 16 & 10.953125 & $1.832487 \times 10^{-6}$ & 15 & 14.984375 \\
\hline
\end{tabular}


Novamente, podemos ver das tabelas (4.18) e (4.19) que o método HIA proporciona melhores resultados quando comparado com o método HA, no entanto este método demanda maior tempo computacional.

O problema (4) também é resolvido numericamente usando a condição de fronteira tipo Neumann a qual é obtida da solução analítica. Os seguintes resultados são relativos ao método HIA. Para resolver o sistema linear usamos o método Bi-PGC com a fatorização incompleta LU com drop-tolerance $=10^{-3}$ onde obtemos:

Tabela 4.20 Aproximação aplicando o método HIA do Problema 4 usando o erro relativo na norma do máximo e condição de fronteira tipo Neumann com drop tolerance.

\begin{tabular}{|c|c|c|c|}
\hline GRID & \multicolumn{3}{|c|}{ HIA } \\
\hline N=M & $\begin{array}{c}\text { Erro relativo na } \\
\text { norma do } \\
\text { máximo }\end{array}$ & Iter & Tempo \\
\hline 12 & $8.821918 \times 10^{-4}$ & 2 & 0.1875 \\
\hline 22 & $3.460102 \times 10^{-4}$ & 2 & 0.265625 \\
\hline 42 & $9.544108 \times 10^{-5}$ & 2 & 0.53125 \\
\hline 82 & $2.505263 \times 10^{-5}$ & 3 & 2.328125 \\
\hline 162 & $5.751978 \times 10^{-6}$ & 5 & 15.390625 \\
\hline
\end{tabular}

O critério de parada foi a tolerância relativa $t o l=10^{-6}$ e como podemos observar na tabela 4.20 os erros relativos na norma do máximo e o tempo computacional foram parecidos daqueles mostrados na tabela 4.19 onde foi considerado a condição de fronteira tipo Dirichlet.

\subsubsection{Solução numérica da equação de difusão com coeficientes descontínuos em coordenadas polares}

Consideramos novamente a equação de difusão (4.1) dada em coordenadas polares junto às condições de fronteira tipo Dirichlet ou Neumann (4.2) e o domínio $\Omega$ como sendo o disco unitário e os coeficientes $\sigma(r, \theta)$ descontínuos e constantes por partes.

A malha a ser usada é a mesma das figuras 4.1, 4.2 e 4.3.

\section{Interfaces}

Analogamente ao caso em que as coordenadas são retangulares consideramos às interfaces sendo ortogonais aos eixos coordenados. 
Inicialmente vamos alinhar as curvas das interfaces com os pontos médios entre dois pontos da malha (laterais dos volumes finitos). Porém, de uma maneira geral, as interfaces podem estar localizadas em qualquer lugar entre dois nós da malha.

Denotaremos um volume finito genérico da forma: $V_{P}=[i-1 / 2, i+1 / 2] \times[j-1 / 2, j+1 / 2]$.

Na Figura 4.13 é mostrado um volume finito genérico.

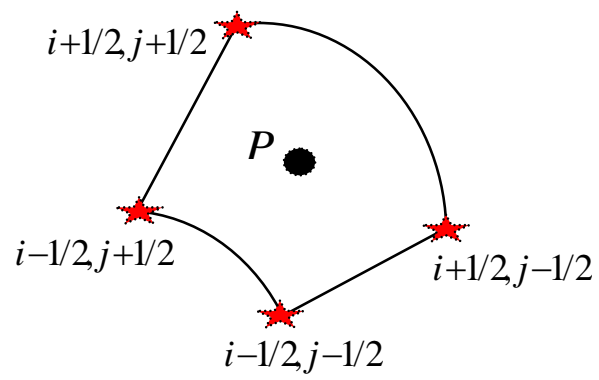

Figura 4.13 Um volume finito em coordenas polares.

\section{Interfaces no eixo radial:}

Consideramos a interface $\xi$ em algum lugar da célula $\left[r_{i}, r_{i+1}\right]$ do eixo radial. Assim denotamos como $\xi=r_{i}+v h_{r}$, onde $v \in[0,1]$.

\section{Interfaces no eixo angular:}

Da mesma maneira, denotamos as interfaces no eixo angular localizados no intervalo $\left[\theta_{j}, \theta_{j+1}\right]$ como $\eta=\theta_{j}+v h_{\theta}$, onde $v \in[0,1]$.

Assim temos os seguintes domínios: físicos e computacional.

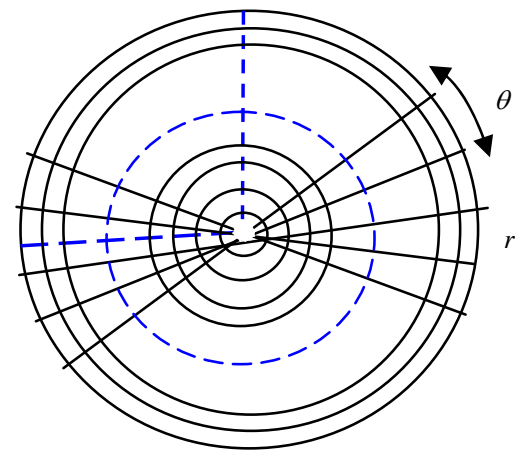

(a)

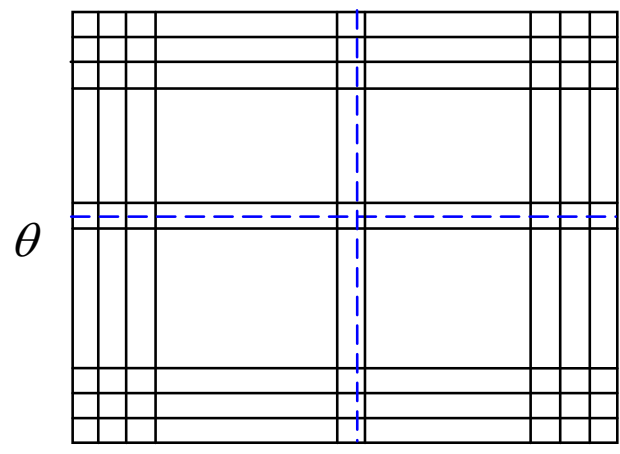

(b)

Figura 4.14 (a) Domínio físico, (b) Domínio computacional. 


\section{Discretização da equação de difusão}

O fluxo do problema (4.1) tem a forma:

$$
W(r, \theta)=-\sigma(r, \theta) \nabla u(r, \theta)=\left[\begin{array}{c}
-\sigma u_{r} \\
-\frac{\sigma}{r} u_{\theta}
\end{array}\right]=\left[\begin{array}{l}
W_{1} \\
W_{2}
\end{array}\right]
$$

Nesse termos a equação de difusão fica:

$$
\nabla \cdot W(r, \theta)=f(r, \theta)
$$

Integrando a equação em $V_{p}$ temos:

$$
\int_{V_{p}} \nabla \cdot W d A=r h_{r} h_{\theta} \varphi_{p}, \quad \varphi_{p}=\frac{1}{r h_{r} h_{\theta}} \int_{V_{p}} f d A,
$$

e pelo teorema da divergência:

$$
\int_{V_{p}} \nabla \cdot W d A=\int_{\partial V_{p}} W \cdot n d l
$$

então:

$$
\left[-\int_{\theta_{j-1 / 2}}^{\theta_{j+1 / 2}}\left(r_{i+1 / 2}\left(\sigma u_{r}\right)\left(r_{i+1 / 2}, \theta\right)-r_{i-1 / 2}\left(\sigma u_{r}\right)\left(r_{i-1 / 2}, \theta\right)\right) d \theta\right]+\left[-\int_{r_{i-1 / 2}}^{r_{i+1 / 2}}\left(\left(\frac{1}{r} \sigma u_{\theta}\right)\left(r, \theta_{j+1 / 2}\right)-\left(\frac{1}{r} \sigma u_{\theta}\right)\left(r, \theta_{j-1 / 2}\right)\right) d r\right]=h_{r} h_{\theta} r_{i} \varphi_{P}
$$

e agora, aproximando as integrais pela regra do ponto médio, temos:

$$
-\left\{h_{\theta}\left[r_{i+1 / 2}\left(\sigma u_{r}\right)_{i+1 / 2, j}-r_{i-1 / 2}\left(\sigma u_{r}\right)_{i-1 / 2, j}\right]+h_{r}\left[\left(\frac{\sigma}{r} u_{\theta}\right)_{i, j+1 / 2}-\left(\frac{\sigma}{r} u_{\theta}\right)_{i, j-1 / 2}\right]\right\}=h_{r} h_{\theta} r_{i} f_{i, j}
$$

$\mathrm{ou}$

$$
h_{\theta}\left[r_{i+1 / 2}\left(W_{1}\right)_{i+1 / 2, j}-r_{i-1 / 2}\left(W_{1}\right)_{i-1 / 2, j}\right]+h_{r}\left[\left(W_{2}\right)_{i, j+1 / 2}-\left(W_{2}\right)_{i, j-1 / 2}\right]=h_{r} h_{\theta} r_{i} f_{i, j}
$$


Esquemas da media harmônica e da media harmônica modificada em coordenadas polares

Das aproximações das componentes do fluxo $W_{1}, W_{2}$ pela serie de Taylor são obtidos os métodos dos volumes finitos que usa a media harmônica HA, e o método que usa uma media harmônica modificada HIA.

\section{Esquema HA}

\section{Discretização na direção radial:}

Sabendo que $W_{1}=-\sigma u_{r} \rightarrow-u_{r}=\frac{W_{1}}{\sigma}$ e aproximando $W_{1}(r, \theta)$ pela série de Taylor truncada antes dos termos de primeira ordem temos: $W_{1}(r, \theta) \approx\left(W_{1}\right)_{i+1 / 2 j}$ então:

$$
-\int_{r_{i}}^{r_{i+1}} u_{r} d r=\int_{r_{i}}^{r_{i+1}} \frac{W_{1}(r, \theta)}{\sigma(r, \theta)} d r=\left(W_{1}\right)_{i+1 / 2} \int_{r_{i}}^{r_{i+1}} \frac{d r}{\sigma(r, \theta)}+O\left(h_{r}^{2}\right)
$$

e

$$
-r_{i+1 / 2} \frac{u_{i+1, j}-u_{i, j}}{h_{r}}=r_{i+1 / 2}\left(W_{1}\right)_{i+1 / 2, j} \frac{1}{h_{r}} \int_{r_{i}}^{r_{i+1}} \frac{d r}{\sigma(r, \theta)}+O\left(h_{r}\right) .
$$

Assim:

$$
-r_{i+1 / 2} K_{i+1 / 2, j}^{H} \frac{u_{i+1, j}-u_{i, j}}{h_{r}}=r_{i+1 / 2}\left(W_{1}\right)_{i+1 / 2, j},
$$

onde:

$$
K_{i+1 / 2, j}^{H}=\left(\frac{1}{h_{r}} \int_{r_{i}}^{r_{i+1}} \frac{d r}{\sigma\left(r, \theta_{j}\right)}\right)^{-1} .
$$

Da mesma maneira, temos que:

$$
-r_{i-1 / 2} K_{i-1 / 2, j}^{H} \frac{u_{i, j}-u_{i-1, j}}{h_{r}}=r_{i-1 / 2}\left(W_{1}\right)_{i-1 / 2, j},
$$

onde: 


$$
K_{i-1 / 2, j}^{H}=\left(\frac{1}{h_{r}} \int_{r_{i-1}}^{r_{i}} \frac{d r}{\sigma\left(r, \theta_{j}\right)}\right)^{-1} .
$$

Portanto:

$$
\left\{\begin{array}{l}
-r_{i+1 / 2} K_{i+1 / 2, j}^{H} \frac{u_{i+1, j}-u_{i, j}}{h_{r}}=r_{i+1 / 2}\left(W_{1}\right)_{i+1 / 2, j} \\
-r_{i-1 / 2} K_{i-1 / 2, j}^{H} \frac{u_{i, j}-u_{i-1, j}}{h_{r}}=r_{i-1 / 2}\left(W_{1}\right)_{i-1 / 2, j}
\end{array}\right.
$$

Em conseqüência a discretização da equação (4.1) na direção radial pelo método HA fica:

$$
-\left[r_{i+1 / 2} K_{i+1 / 2, j}^{H} \frac{u_{i+1, j}-u_{i, j}}{h_{r}}-r_{i-1 / 2} K_{i-1 / 2, j}^{H} \frac{u_{i, j}-u_{i-1, j}}{h_{r}}\right]=r_{i+1 / 2}\left(W_{1}\right)_{i+1 / 2, j}-r_{i-1 / 2}\left(W_{1}\right)_{i-1 / 2, j}
$$

\section{Discretização na direção angular:}

Sabendo que $W_{2}=-\frac{\sigma}{r} u_{\theta} \rightarrow-\frac{u_{\theta}}{r}=\frac{W_{2}}{\sigma}$ e aproximando $W_{2}(r, \theta)$ pela série de Taylor truncada antes dos termos de primeira ordem:

$$
W_{1}(r, \theta)=\left(W_{1}\right)_{i+1 / 2 j},
$$

então:

$$
-\int_{\theta_{j}}^{\theta_{j+1}} \frac{u_{\theta}}{r} d \theta=\int_{\theta_{j}}^{\theta_{j+1}} \frac{W_{2}(r, \theta)}{\sigma(r, \theta)} d \theta=\left(W_{2}\right)_{i j+1 / 2} \int_{\theta_{j}}^{\theta_{j+1}} \frac{d \theta}{\sigma(r, \theta)}
$$

e

$$
-\frac{u_{i, j+1}-u_{i, j}}{r_{i} h_{\theta}}=\theta_{j+1 / 2}\left(W_{2}\right)_{i, j+1 / 2} \frac{1}{h_{\theta}} \int_{\theta_{j}}^{\theta_{j+1}} \frac{d \theta}{\sigma(r, \theta)} .
$$

Assim:

$$
-K_{i, j+1 / 2}^{H} \frac{u_{i, j+1}-u_{i, j}}{r_{i} h_{\theta}}=\left(W_{2}\right)_{i, j+1 / 2}
$$

onde: 


$$
K_{i, j+1 / 2}^{H}=\left(\frac{1}{h_{\theta}} \int_{\theta_{j}}^{\theta_{j+1}} \frac{d \theta}{\sigma\left(r_{i}, \theta\right)}\right)^{-1} .
$$

Da mesma maneira:

$$
-K_{i, j-1 / 2}^{H} \frac{u_{i, j}-u_{i, j-1}}{r_{i} h_{\theta}}=\left(W_{2}\right)_{i, j-1 / 2},
$$

onde:

$$
K_{i, j-1 / 2}^{H}=\left(\frac{1}{h_{\theta}} \int_{\theta j-1}^{\theta_{j}} \frac{d \theta}{\sigma\left(r_{i}, \theta\right)}\right)^{-1} .
$$

Portanto:

$$
\left\{\begin{array}{l}
-K_{i, j-1 / 2}^{H} \frac{u_{i, j+1}-u_{i, j}}{r_{i} h_{\theta}}=\left(W_{2}\right)_{i, j+1 / 2} \\
-K_{i, j-1 / 2}^{H} \frac{u_{i, j}-u_{i, j-1}}{r_{i} h_{\theta}}=\left(W_{2}\right)_{i, j-1 / 2}
\end{array} .\right.
$$

Assim, temos que a discretização da equação (4.1) na direção angular pelo método HA fica:

$$
-\left[K_{i, j+1 / 2}^{H} \frac{u_{i, j+1}-u_{i, j}}{r_{i} h_{\theta}}-K_{i, j-1 / 2}^{H} \frac{u_{i, j}-u_{i, j-1}}{r_{i} h_{\theta}}\right]=\left(W_{2}\right)_{i, j+1 / 2}-\left(W_{2}\right)_{i, j-1 / 2} .
$$

Também é possível aplicar a metodologia dos esquemas HA e HIA em problemas onde precisamos ter à aproximação no pólo $(r=0)$. Para isso, precisamos considerar o pólo como sendo parte da malha e fazer algumas considerações especiais, como descritos a seguir:

Dividimos $V_{1}=[0,1+1 / 2] \times[j-1 / 2, j+1 / 2]$ como mostra a figura 4.15 , em duas regiões de integração, de $r=0$ a $r=\frac{1}{4} h_{r}$ e de $r=\frac{1}{4} h_{r}$ a $r=h_{r}$. 
Na direção radial $r=\frac{1}{4} h_{r}$ a $r=h_{r}$ consideramos o intervalo radial centrado em $r_{a}=\frac{5 h_{r}}{8}$. Assim os limites de integração são:

$$
\left\{\begin{array}{l}
r_{a}+\frac{3 h_{r}}{8} \\
r_{a}-\frac{3 h_{r}}{8}
\end{array} .\right.
$$

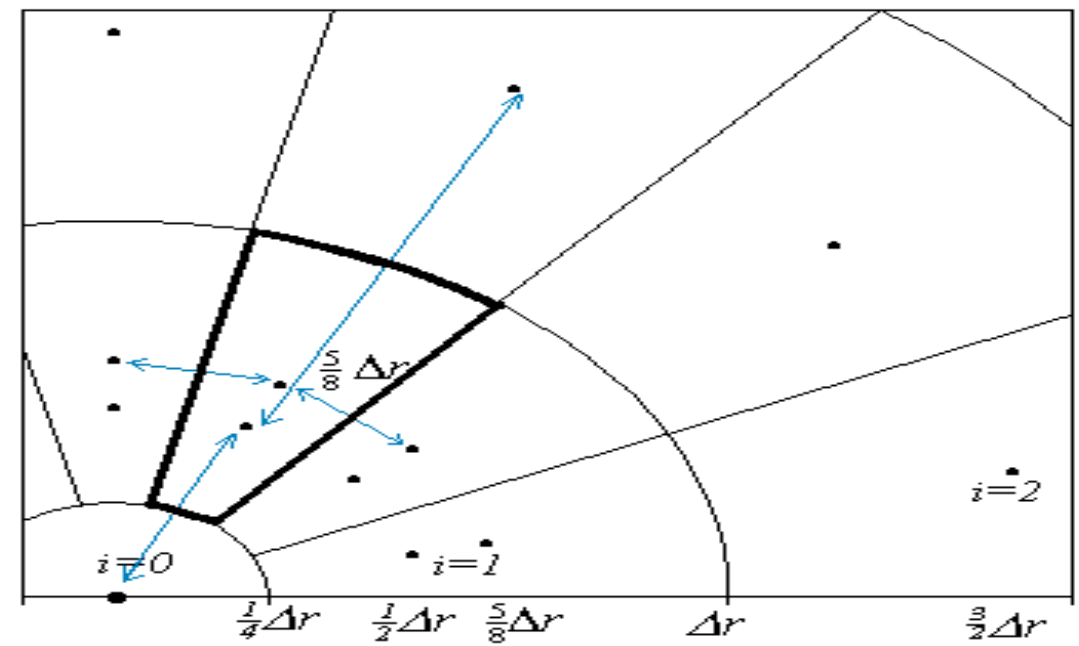

Figura 4.15 Malha perto do pólo [68].

De:

$$
\int_{V_{1}=V_{0}+V_{a}} \nabla \cdot \bar{W} d A=r h_{r} h_{\theta} \varphi, \quad \varphi=\frac{1}{r h_{r} h_{\theta}} \int_{V} f d A
$$

chegamos a:

$$
\left\{h_{\theta}\left[r_{a+3 h_{r} / 8}\left(W_{1}\right)_{a+3 h_{r} / 8 j}-r_{a-3 h_{r} / 8}\left(W_{1}\right)_{a-3 h_{r} / 8 j}\right]+\frac{3 h_{r}}{8}\left[\left(W_{2}\right)_{a j+1 / 2}-\left(W_{2}\right)_{a j-1 / 2}\right]\right\}=-r_{1} f_{1 j} .
$$

Assim:

$$
h_{\theta}\left[h_{r} K_{1+1 / 2, j}^{H} \frac{u_{2, j}-u_{1, j}}{h_{r}}-\frac{h_{r}}{4} K_{1-1 / 2, j}^{H} \frac{u_{1, j}-u_{0, j}}{h_{r} / 2}\right]+\frac{3 h_{r}}{4}\left[K_{1, j+1 / 2}^{H} \frac{u_{a, j+1}-u_{a, j}}{r_{a} h_{\theta}}-K_{1, j-1 / 2}^{H} \frac{u_{a, j}-u_{a, j-1}}{r_{a} h_{\theta}}\right]=-r_{1} f_{1, j},
$$


e fazendo interpolação linear:

$$
u_{a, j}=\frac{1}{8}\left(7 u_{1, j}+u_{2, j}\right), u_{a, j+1}=\frac{1}{8}\left(7 u_{1, j+1}+u_{2, j+1}\right) u_{a, j-1}=\frac{1}{8}\left(7 u_{1, j-1}+u_{2, j-1}\right) .
$$

Assim: Para $i=1,1 \leq j \leq N$ :

$$
\begin{aligned}
& \left(\frac{21}{20} \frac{K_{1, j-1 / 2}^{H}}{h_{\theta}}\right) u_{1, j-1}-\left[h_{\theta} K_{3 / 2, j}^{H}+\frac{K_{1 / 2, j}^{H}}{2} h_{\theta}+\frac{21}{20} \frac{K_{1, j+1 / 2}^{H}}{h_{\theta}}+\frac{21}{20} \frac{K_{1, j-1 / 2}^{H}}{h_{\theta}}\right] u_{1, j}+\left(\frac{21}{20} \frac{K_{1, j+1 / 2}^{H}}{h_{\theta}}\right) u_{1, j+1}+ \\
& {\left[h_{\theta} K_{3 / 2, j}^{H}-\frac{3}{20} \frac{K_{1, j+1 / 2}^{H}}{h_{\theta}}-\frac{3}{20} \frac{K_{1, j-1 / 2}^{H}}{h_{\theta}}\right] u_{2, j}+\left(\frac{3}{20} \frac{K_{1, j-1 / 2}^{H}}{h_{\theta}}\right) u_{2, j-1}+\left(\frac{3}{20} \frac{K_{1, j+1 / 2}^{H}}{h_{\theta}}\right) u_{2, j+1}+} \\
& \frac{K_{1 / 2, j}^{H}}{2} h_{\theta} u_{o, j}=-r_{1} f_{1, j}
\end{aligned}
$$

\section{O sistema linear}

\section{Na direção radial:}

Para $i=1,1 \leq j \leq N$ :

$$
r_{1+1 / 2}\left(W_{1}\right)_{1+1 / 2, j}=-r_{1+1 / 2} K_{1+1 / 2, j}^{H} \frac{u_{2, j}-u_{1, j}}{h_{r}}
$$

Para $2 \leq i \leq M-1,1 \leq j \leq N$ :

$$
r_{i+1 / 2}\left(W_{1}\right)_{i+1 / 2, j}-r_{i-1 / 2}\left(W_{1}\right)_{i-1 / 2, j}=-\left[r_{i+1 / 2} K_{i+1 / 2, j}^{H} \frac{u_{i+1, j}-u_{i, j}}{h_{r}}-r_{i-1 / 2} K_{i-1 / 2, j}^{H} \frac{u_{i, j}-u_{i-1, j}}{h_{r}}\right]
$$

Para $i=M, 1 \leq j \leq N$ :

$$
r_{M+1 / 2}\left(w_{1}\right)_{M+1 / 2, j}-r_{M-1 / 2}\left(w_{1}\right)_{M-1 / 2, j}=-\left[r_{M+1 / 2} K_{M+1 / 2, j}^{H} \frac{g_{j}-u_{M, j}}{h_{r}}-r_{M-1 / 2} K_{M-1 / 2, j}^{H} \frac{u_{M, j}-u_{M-1, j}}{h_{r}}\right] .
$$

Considerando os coeficientes $\sigma(r, \theta)$ contínuos por partes temos: 


$$
\left\{\begin{array}{l}
K_{i+1 / 2, j}^{H}=2 \frac{\sigma_{i+1, j} \sigma_{i, j}}{\sigma_{i+1, j}+\sigma_{i, j}}=, i=1, M \\
K_{i-1 / 2, j}^{H}=2 \frac{\sigma_{i-1, j} \sigma_{i, j}}{\sigma_{i-1, j}+\sigma_{i, j}}, i=2, M+1
\end{array}\right.
$$

\section{Aproximação nas interfaces}

Em geral se a interface se encontra no intervalo $\left[r_{i}, r_{i+1}\right]$ e aproximando os valores de $K_{i+1 / 2 j}^{H}$ podem ser aproximados pelo método do ponto médio.

Seja:

$$
\xi=r_{i}+v h_{r}, 0 \leq v \leq 1
$$

uma interface no eixo radial e:

$$
K_{i+1 / 2, j}^{H}=\left(\frac{1}{h_{r}} \int_{r_{i}}^{r_{i+1}} \frac{d r}{\sigma\left(r, \theta_{j}\right)}\right)^{-1},
$$

onde temos que considerar a interface nessa integral. Em particular se a interface se encontra no ponto médio do intervalo $\left[r_{i}, r_{i+1}\right]$, aproximamos os valores de $K_{i+1 / 2 j}^{H}$ pelo método do ponto médio, assim:

$$
\frac{1}{h_{r}} \int_{r_{i}}^{r_{i}+1} \frac{d r}{\sigma(r, \theta)}=\frac{1}{h_{r}}\left[\frac{1}{\sigma_{i, j}}\left(\int_{r_{i}}^{r_{i+1 / 2}} d r\right)+\frac{1}{\sigma_{i+1, j}}\left(\int_{r_{i+1 / 2}}^{r_{i+1}} d r\right)\right],
$$

então:

$$
K_{i+1 / 2, j}^{H}=2\left(\frac{\sigma_{i, j} \sigma_{i+1, j}}{\sigma_{i, j}+\sigma_{i+1, j}}\right)
$$

Na direção angular:

Para $1 \leq j \leq N, \quad 1 \leq i \leq M$

$$
-\left[K_{i, j+1 / 2}^{H} \frac{u_{i, j+1}-u_{i, j}}{r_{i} h_{\theta}}-K_{i, j-1 / 2}^{H} \frac{u_{i, j}-u_{i, j-1}}{r_{i} h_{\theta}}\right]=\left(W_{2}\right)_{i, j+1 / 2}-\left(W_{2}\right)_{i, j-1 / 2} .
$$


Nos intervalos $\left[\theta_{j}, \theta_{j+1}\right]$ considerando os coeficientes $\sigma(r, \theta)$ contínuos por partes, os valores de $K_{i, j+1 / 2}^{H}$ são:

$$
\left\{\begin{array}{l}
K_{i, j+1 / 2}^{H}=2 \frac{\sigma_{i, j} \sigma_{i, j+1}}{\sigma_{i, j}+\sigma_{i, j+1}}, j=1, N \\
K_{i, j-1 / 2}^{H}=2 \frac{\sigma_{i, j} \sigma_{i, j-1}}{\sigma_{i, j}+\sigma_{i, j-1}}, \quad j=2, N
\end{array}\right.
$$

\section{Aproximação nas interfaces:}

Nos intervalos $\left[\theta_{j}, \theta_{j+1}\right]$, os valores de $K_{i+1 / 2, j}^{H}$ são aproximados como:

$$
K_{i, j+1 / 2}^{H}=2\left(\frac{\sigma_{i, j} \sigma_{i, j+1}}{\sigma_{i, j}+\sigma_{i, j+1}}\right) .
$$

Montagem da Matriz $A G$ do sistema linear $(A G) * U=b$

Substituindo (4.50) e (4.59) em (4.42) e considerando $A(i, j), B(i, j), C(i, j)$ como sendo os coeficientes das variáveis $u_{i j}$ em (4.42), montamos o sistema linear $(A G) * U=b$ correspondente ao esquema HA.

Os primeiros blocos da matriz $A G$ no caso em que não se considera o pólo:

Para $i=1, \quad j=1, N$

$$
\left\{\begin{array}{l}
C(1,1) u_{11}-B(1,1) u_{1,2}-B(1) u_{1, N}-A(1,1) u_{2,1}=r_{1} f_{1,1} \\
-B(i, j-1) u_{i, j-1}+C(1, j) u_{1, j}-B(1, j) u_{1, j+1}-A(1, j) u_{2, j}=r_{1} f_{1, j}, j=2, N-1 \\
-B(1, N) u_{1,1}-B(1, N-1) u_{1, N-1}+C(1, N) u_{1, N}-A(1, N) u_{2, N}=r_{1} f_{1, N}
\end{array}\right.
$$

Assim temos os primeiros blocos de $A G$

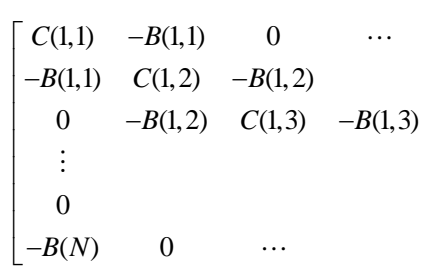

$$
\begin{array}{cccccccc} 
& 0 & -B(1) & -A(1,1) & 0 & \cdots & & 0 \\
& & 0 & 0 & -A(1,2) & & & \\
\ddots & & \vdots & \vdots & & -A(1,3) & & \vdots \\
-B(1, N-2) & C(1, N-1) & -B(1, N-1) & & & & \ddots & \\
0 & -B(1, N-1) & C(1, N) & 0 & \cdots & & 0 & -A(1, N-1) \\
\end{array}
$$

Para $i=2, M-1 \quad j=1, N$ 


$$
\begin{aligned}
& \left\{\begin{array}{l}
-A(i-1,1) u_{i-1,1}+C(i, 1) u_{i, 1}-B(i, 1) u_{i, 2}-B(i) u_{i, N}-A(i, 1) u_{i+1,1}=r_{i} f_{i, 1} \\
-A(i-1, j) u_{i-1, j}-B(i, j-1) u_{i, j-1}+C(i, j) u_{i, j}-B(i, j) u_{i, j+1}-A(i, j) u_{i+1, j}=r_{i} f_{i, j}, j=2, N-1 \\
-A(i-1, N) u_{i-1, N}-B(i) u_{i, 1}-B(i, N-1) u_{i, N-1}+C(i, N) u_{i, N}-A(i, N) u_{i+1, N}=r_{i} f_{i, N}
\end{array}\right.
\end{aligned}
$$

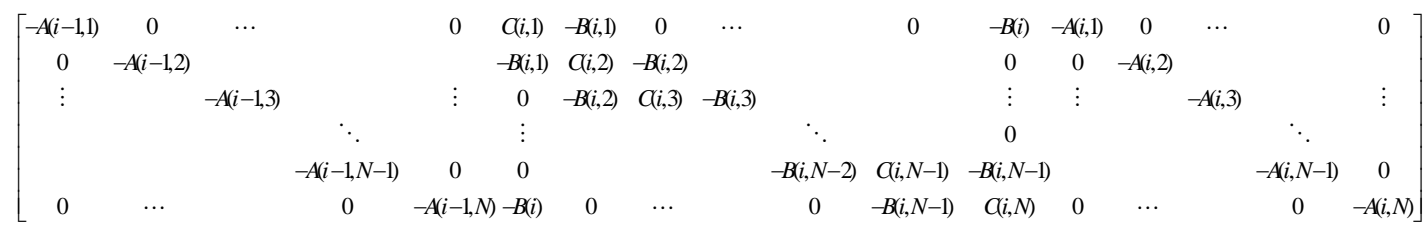

Para $i=M \quad j=1, N$

$$
\left\{\begin{array}{l}
-A(M-1,1) u_{M-1,1}+C(M, 1) u_{M, 1}-B(M, 1) u_{M, 2}-B(M) u_{M, N}=r_{M} f_{M, 1}+A(M, 1) g(1) \\
-A(M-1, j) u_{M-1, j}-B(M, j-1) u_{M, j-1}+C(M, j) u_{M, j}-B(M, j) u_{M, j+1}=r_{M} f_{M, j}+A(M, j) g(j), j=2, N-1 \\
-A(M-1, N) u_{M-1, N}-B(M) u_{M, 1}-B(M, N-1) u_{M, N-1}+C(M, N) u_{M, N}=r_{1} f_{1, N}+A(M, N) g(N)
\end{array},\right.
$$

assim os últimos blocos de $A G$ são:

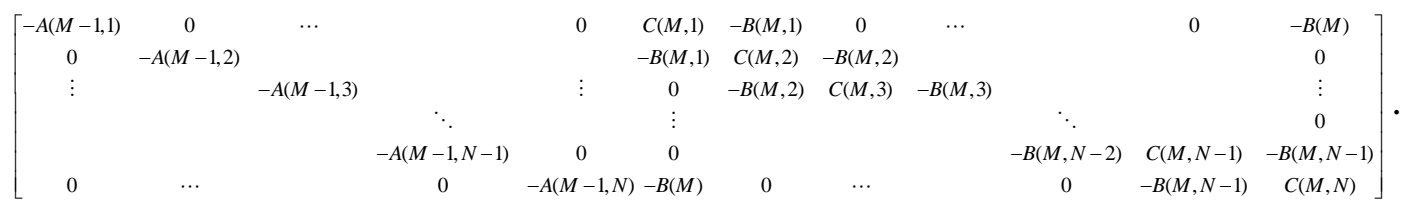

Portanto, a matriz $A G$ (em blocos) fica da seguinte forma:

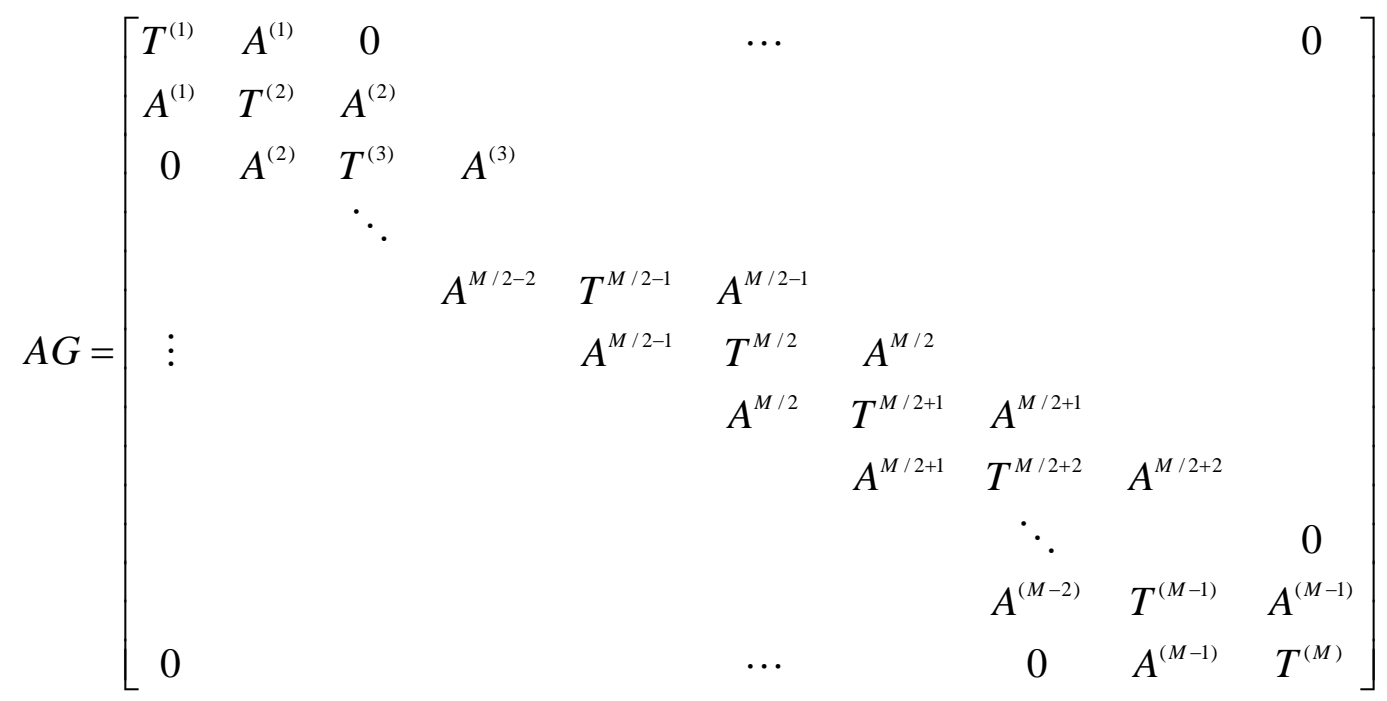


A matriz $A G$ é simétrica e definida positiva, assim o sistema $\left(A G^{*} U\right)=b$ tem uma solução única.

Os primeiros blocos da matriz AG quando consideramos o pólo fica:

Para $i=1, \quad j=1, N$

$$
\left\{\begin{array}{l}
-z 0(1) u_{0,1}+C(1,1) u_{1,1}-B(1,1) u_{1,2}-B(1) u_{1, N}-D(1) u_{2,1}-A(1) u_{2,2}-E 0 u_{2, N}=r_{1} f_{1,1} \\
-z 0(i) u_{0, i}-B(i, j-1) u_{i, j-1}+C(1, j) u_{1, j}-B(1, j) u_{1, j+1}-A(j-1) u_{2, j-1}-D(i) u_{2, j}-A(j) u_{2, j+1}=r_{1} f_{1, j}, j=2, N-1 \\
-z 0(N) u_{0, N}-B(1, N) u_{1,1}-B(1, N-1) u_{1, N-1}+C(1, N) u_{1, N}-A(N) u_{2,1}-A(N-1) u_{2, N-1}-D(N) u_{2, N}=r_{1} f_{1, N}
\end{array}\right.
$$

Assim temos os primeiros blocos de $A G$

\begin{tabular}{|c|c|c|c|c|c|c|c|c|c|c|}
\hline$c 0$ & $-z 0(1)$ & $-z 0(2)$ & $-z 0(3)$ &.. & $-z 0(N)$ & 0 & 0 & & $\ldots$ & 0 \\
\hline$-z 0(1)$ & $C(1,1)$ & $-B(1,1)$ & & & $-B(1)$ & $-D(1)$ & $-A(1)$ & 0 & & $-A 0$ \\
\hline \multirow[t]{4}{*}{$-z 0(2)$} & $-B(1,1)$ & $C(1,2)$ & $-B(1,2)$ & & & $-A(1)$ & $-D(2)$ & $-A(2)$ & & \\
\hline & & & & & & 0 & & & & \\
\hline & & & & $\cdot$ & & & & & $\ddots$ & 0 \\
\hline & & & & & $-B(1, N-1)$ & 0 & & & & $-A(N-1)$ \\
\hline$-z 0(N)$ & $B(1)$ & & & $-B(1, N-1)$ & $C(1, N)$ & A0 & 0 & & $0 \quad A(N-1)$ & $D(N)$ \\
\hline
\end{tabular}

\section{Esquema HIA}

\section{Discretização na direção radial:}

Sabendo que $W_{1}=-\sigma u_{r} \rightarrow-u_{r}=\frac{W_{1}}{\sigma}$ e aproximando $W_{1}(r, \theta)$ pela série de Taylor até os termos de primeira ordem:

$$
W_{1}(r, \theta)=\left(W_{1}\right)_{i+1 / 2, j}+\left(r-r_{i+1 / 2}\right)\left[\left(W_{1}\right)_{r}\right]_{i+1 / 2, j}+O\left(h_{r}^{2}\right)
$$

então:

$$
\begin{gathered}
-\int_{r_{i}}^{r_{i+1}} u_{r} d r=\int_{r_{i}}^{r_{i+1}} \frac{W_{1}(r, \theta)}{\sigma(r, \theta)} d r=\left(W_{1}\right)_{i+1 / 2, j} \int_{r_{i}}^{r_{i+1}} \frac{d r}{\sigma(r, \theta)}+\left[\left(W_{1}\right)_{r}\right]_{i+1 / 2, j} \int_{r_{i}}^{r_{i+1}} \frac{r-r_{i+1 / 2}}{\sigma(r, \theta)} d r+O\left(h_{r}^{3}\right) \mathrm{e} \\
-r_{i+1 / 2} \frac{u_{i+1, j}-u_{i, j}}{h_{r}}=r_{i+1 / 2}\left(W_{1}\right)_{i+1 / 2, j} \frac{1}{h_{r}} \int_{r_{i}}^{r_{i+1}} \frac{d r}{\sigma(r, \theta)}+r_{i+1 / 2}\left[\left(W_{1}\right)_{r}\right]_{i+1 / 2, j} \frac{1}{h_{r}} \int_{r_{i}}^{r_{i+1}} \frac{r-r_{i+1 / 2}}{\sigma(r, \theta)} d r+O\left(h_{r}{ }^{2}\right)
\end{gathered}
$$

Fazendo $\left(r W_{1}\right)_{r}=r\left(W_{1}\right)_{r}+W_{1}$, então: 


$$
r\left(W_{1}\right)_{r}=\left(r W_{1}\right)_{r}-W_{1} \text {, }
$$

temos que:

$$
\begin{aligned}
& -r_{i+1 / 2} \frac{u_{i+1, j}-u_{i, j}}{h_{r}}=r_{i+1 / 2}\left(W_{1}\right)_{i+1 / 2, j} \frac{1}{h_{r}} \int_{r_{i}}^{r_{i+1}}\left(\frac{1}{\sigma(r, \theta)}-\frac{r-r_{i+1 / 2}}{r_{i+1 / 2} \sigma(r, \theta)}\right) d r+ \\
& +\left[r_{i+1 / 2}\left(w_{1}\right)_{i+1 / 2, j}-r_{i-1 / 2}\left(W_{1}\right)_{i-1 / 2, j}\right]_{i+1 / 2, j} \frac{1}{h_{r}^{2}} \int_{r_{i}}^{r_{i+1}} \frac{r-r_{i+1 / 2}}{\sigma(r, \theta)} d r+O\left(h_{r}^{2}\right)
\end{aligned}
$$

Assim:

$$
-r_{i+1 / 2} K_{i+1 / 2, j}^{H} \frac{u_{i+1, j}-u_{i, j}}{h_{r}}=r_{i+1 / 2}\left(W_{1}\right)_{i+1 / 2, j}+\left[r_{i+1 / 2}\left(W_{1}\right)_{i+1 / 2, j}-r_{i-1 / 2}\left(W_{1}\right)_{i-1 / 2, j}\right] a_{i+1 / 2, j},
$$

onde

$$
\begin{gathered}
K_{i+1 / 2, j}^{H}=\left(\frac{1}{h_{r}} \int_{r_{i}}^{r_{i+1}}\left(1-\frac{\left(r-r_{i+1 / 2}\right)}{r_{i+1 / 2}}\right) \frac{d r}{\sigma\left(r, \theta_{j}\right)}\right)^{-1} \\
a_{i+1 / 2, j}=K_{i+1 / 2, j}^{H} \frac{1}{h_{r}^{2}} \int_{r_{i}}^{r_{i+1}} \frac{r-r_{i+1 / 2}}{\sigma\left(r, \theta_{j}\right)} d r
\end{gathered}
$$

sendo que os limites do termo $1-\frac{\left(r-r_{i+1 / 2}\right)}{r_{i+1 / 2}}$ são:

$$
r_{i} \leq r \leq r_{i+1} \rightarrow 0 \leq 1-\frac{r-r_{i+1 / 2}}{r_{i+1 / 2}} \leq 1+\frac{h_{r}}{2 r_{i+1 / 2}}
$$

Da mesma maneira:

$$
-r_{i-1 / 2} K_{i-1 / 2, j}^{H} \frac{u_{i, j}-u_{i-1, j}}{h_{r}}=r_{i-1 / 2}\left(W_{1}\right)_{i-1 / 2, j}+\left[r_{i+1 / 2}\left(W_{1}\right)_{i+1 / 2, j}-r_{i-1 / 2}\left(W_{1}\right)_{i-1 / 2, j}\right] a_{i-1 / 2, j},
$$

onde:

$$
K_{i-1 / 2, j}^{H}=\left(\frac{1}{h_{r}} \int_{r_{i-1}}^{r_{i}}\left(1-\frac{\left(r-r_{i-1 / 2}\right)}{r_{i-1 / 2}}\right) \frac{d r}{\sigma\left(r, \theta_{j}\right)}\right)^{-1},
$$




$$
a_{i-1 / 2, j}=K_{i-1 / 2, j}^{H} \frac{1}{h_{r}^{2}} \int_{r_{i}-1}^{r_{i}} \frac{r-r_{i-1 / 2}}{\sigma\left(r, \theta_{j}\right)} d r
$$

Assim temos que:

$$
\left\{\begin{array}{l}
-r_{i+1 / 2} K_{i+1 / 2, j}^{H} \frac{u_{i+1, j}-u_{i, j}}{h_{r}}=r_{i+1 / 2}\left(W_{1}\right)_{i+1 / 2, j}+\left[r_{i+1 / 2}\left(W_{1}\right)_{i+1 / 2, j}-r_{i-1 / 2}\left(W_{1}\right)_{i-1 / 2, j}\right] a_{i+1 / 2, j} \\
-r_{i-1 / 2} K_{i-1 / 2, j}^{H} \frac{u_{i, j}-u_{i-1, j}}{h_{r}}=r_{i-1 / 2}\left(W_{1}\right)_{i-1 / 2, j}+\left[r_{i-1 / 2}\left(W_{1}\right)_{i+1 / 2, j}-r_{i-1 / 2}\left(w_{1}\right)_{i-1 / 2, j}\right] a_{i-1 / 2, j}
\end{array}\right.
$$

Portanto:

$$
-\left(1+a_{i+1 / 2, j}-a_{i-1 / 2, j}\right)^{-1}\left[r_{i+1 / 2} K_{i+1 / 2, j}^{H} \frac{u_{i+1, j}-u_{i, j}}{h_{r}}-r_{i-1 / 2} K_{i-1 / 2, j}^{H} \frac{u_{i, j}-u_{i-1, j}}{h_{r}}\right]=r_{i+1 / 2}\left(W_{1}\right)_{i+1 / 2, j}-r_{i-1 / 2}\left(W_{1}\right)_{i-1 / 2, j}
$$

\section{Discretização na direção angular:}

Sabendo que $W_{2}=-\frac{\sigma}{r} u_{\theta} \rightarrow-\frac{u_{\theta}}{r}=\frac{W_{2}}{\sigma}$ e aproximando $W_{2}(r, \theta)$ pela série de Taylor ate os termos de primeira ordem:

$$
W_{1}(r, \theta)=\left(W_{1}\right)_{i+1 / 2, j}+\left(r-r_{i+1 / 2}\right)\left[\left(W_{1}\right)_{r}\right]_{i+1 / 2, j}+O\left(h_{r}^{2}\right)
$$

então:

$$
-\int_{\theta_{j}}^{\theta_{j+1}} \frac{u_{\theta}}{r} d \theta=\int_{\theta_{j}}^{\theta_{j+1}} \frac{W_{2}(r, \theta)}{\sigma(r, \theta)} d \theta=\left(W_{2}\right)_{i, j+1 / 2} \int_{\theta_{j}}^{\theta_{j+1}} \frac{d \theta}{\sigma(r, \theta)}+\left[\left(W_{2}\right)_{\theta}\right]_{i, j+1 / 2} \int_{\theta_{j}}^{\theta_{j+1}} \frac{\theta-\theta_{j+1 / 2}}{\sigma(r, \theta)} d \theta+O\left(h_{\theta}{ }^{3}\right),
$$

e:

$$
-\frac{u_{i, j+1}-u_{i, j}}{r_{i} h_{\theta}}=\theta_{j+1 / 2}\left(W_{2}\right)_{i, j+1 / 2} \frac{1}{h_{\theta}} \int_{\theta_{j}}^{\theta_{j+1}} \frac{d \theta}{\sigma(r, \theta)}+\left[\left(W_{2}\right)_{\theta}\right]_{i, j+1 / 2} \frac{1}{h_{\theta}} \int_{\theta_{j}}^{\theta_{j+1}} \frac{\theta-\theta_{j+1 / 2}}{\sigma(r, \theta)} d \theta+O\left(h_{\theta}{ }^{2}\right) .
$$

Assim: 


$$
-K_{i, j+1 / 2}^{H} \frac{u_{i, j+1}-u_{i, j}}{r_{i} h_{\theta}}=\left(W_{2}\right)_{i, j+1 / 2}+\left[\left(W_{2}\right)_{i, j+1 / 2}-\left(W_{2}\right)_{i, j-1 / 2}\right] a_{i, j+1 / 2}
$$

onde:

$$
\begin{gathered}
K_{i, j+1 / 2}^{H}=\left(\frac{1}{h_{\theta}} \int_{\theta_{j}}^{\theta j+1} \frac{d \theta}{\sigma\left(r_{i}, \theta\right)}\right)^{-1}, \\
a_{i, j+1 / 2}=K_{i, j+1 / 2}^{H} \frac{1}{h_{\theta}^{2}} \int_{\theta_{j}}^{\theta+1} \frac{\theta-\theta_{j+1 / 2}}{\sigma\left(r_{i}, \theta\right)} d \theta .
\end{gathered}
$$

Da mesma maneira:

$$
-K_{i, j-1 / 2}^{H} \frac{u_{i, j}-u_{i, j-1}}{r_{i} h_{\theta}}=\left(W_{2}\right)_{i, j-1 / 2}+\left[\left(W_{2}\right)_{i, j+1 / 2}-\left(W_{2}\right)_{i, j-1 / 2}\right] a_{i, j-1 / 2},
$$

onde:

$$
\begin{gathered}
K_{i, j-1 / 2}^{H}=\left(\frac{1}{h_{\theta}} \int_{\theta j-1}^{\theta_{j}} \frac{d \theta}{\sigma\left(r_{i}, \theta\right)}\right)^{-1}, \\
a_{i, j-1}=K_{i, j-1 / 2}^{H} \frac{1}{h_{\theta}^{2}} \int_{\theta j-1}^{\theta j} \frac{\theta-\theta_{j-1 / 2}}{\sigma\left(r_{i}, \theta\right)} d \theta .
\end{gathered}
$$

De:

$$
\left\{\begin{array}{l}
-K_{i, j-1 / 2}^{H} \frac{u_{i, j+1}-u_{i, j}}{r_{i} h_{\theta}}=\left(W_{2}\right)_{i, j+1 / 2}+\left[\left(W_{2}\right)_{i, j+1 / 2}-\left(W_{2}\right)_{i, j-1 / 2}\right] a_{i, j+1 / 2} \\
-K_{i, j-1 / 2}^{H} \frac{u_{i, j}-u_{i, j-1}}{r_{i} h_{\theta}}=\left(W_{2}\right)_{i, j-1 / 2}+\left[\left(W_{2}\right)_{i, j+1 / 2}-\left(W_{2}\right)_{i, j-1 / 2}\right] a_{i j-1 / 2}
\end{array}\right.
$$

temos que:

$$
-\left(1+a_{i, j+1 / 2}-a_{i, j-1 / 2}\right)^{-1}\left[K_{i, j+1 / 2}^{H} \frac{u_{i, j+1}-u_{i, j}}{r_{i} h_{\theta}}-K_{i, j-1 / 2}^{H} \frac{u_{i, j}-u_{i, j-1}}{r_{i} h_{\theta}}\right]=\left(W_{2}\right)_{i, j+1 / 2}-\left(W_{2}\right)_{i, j-1 / 2}
$$




\section{O sistema linear}

\section{Na direção radial:}

Para $i=1,1 \leq j \leq N$ :

$$
r_{1+1 / 2}\left(W_{1}\right)_{1+1 / 2, j}=-\left(1+a_{1+1 / 2, j}\right)^{-1} r_{1+1 / 2} K_{1+1 / 2, j}^{H} \frac{u_{2, j}-u_{1, j}}{h_{r}}
$$

Para $2 \leq i \leq M-1,1 \leq j \leq N$ :

$$
r_{i+1 / 2}\left(W_{1}\right)_{i+1 / 2, j}-r_{i-1 / 2}\left(W_{1}\right)_{i-1 / 2, j}=-\left(1+a_{i+1 / 2, j}-a_{i-1 / 2, j}\right)^{-1}\left[r_{i+1 / 2} K_{i+1 / 2, j}^{H} \frac{u_{i+1, j}-u_{i, j}}{h_{r}}-r_{i-1 / 2} K_{i-1 / 2, j}^{H} \frac{u_{i, j}-u_{i-1, j}}{h_{r}}\right]
$$

Para $i=M, 1 \leq j \leq N$ :

$$
r_{M+1 / 2}\left(W_{1}\right)_{M+1 / 2, j}-r_{M-1 / 2}\left(W_{1}\right)_{M-1 / 2, j}=-\left(1+a_{M+1 / 2, j}-a_{M-1 / 2, j}\right)^{-1}\left[r_{M+1 / 2} K_{M+1 / 2, j}^{H} \frac{g_{j}-u_{M, j}}{h_{r}}-r_{M-1 / 2} K_{M-1 / 2, j}^{H} \frac{u_{M, j}-u_{M-1, j}}{h_{r}}\right] .
$$

Nos intervalos $\left[r_{i}, r_{i+1}\right], i=1, \ldots, M / 2-1, \quad i=M / 2+1, \ldots, M \quad$ consideramos os coeficientes $\sigma(r, \theta)$ contínuos por partes, assim os valores de $K_{i+1 / 2 j}^{H}, a_{i+1 / 2 j}$ podem ser obtidos com seus valores exatos:

$$
\begin{gathered}
\left\{\begin{array}{l}
K_{i+1 / 2, j}^{H}=2 r_{i+1 / 2} \frac{\sigma_{i, j} \sigma_{i+1, j}}{\sigma_{i, j} r_{i+1 / 4}+\sigma_{i+1, j} r_{i+3 / 4}}, i=1, M \\
K_{i-1 / 2, j}^{H}=K_{(i-1)+1 / 2, j}^{H}, i=2, M+1
\end{array},\right. \\
\left\{\begin{array}{l}
a_{i+1 / 2, j}=0, i=1, M / 2-1 ; i=M / 2+1, M \\
a_{i-1 / 2, j}=0, i=2, M / 2 ; i=M / 2+2, M+1
\end{array}\right.
\end{gathered}
$$

\section{Aproximação nas interfaces:}

Considerando a interface no ponto médio do intervalo $\left[r_{i}, r_{i+1}\right]$, os valores de $K_{i+1 / 2, j}^{H}, a_{i+1 / 2, j}$ podem se aproximados pelo método do ponto médio, então:

$$
\frac{1}{h_{r}} \int_{r_{i}}^{r_{i+1}}\left(1-\frac{r-r_{i+1 / 2}}{r_{i+1 / 2}}\right) \frac{d r}{\sigma(r, \theta)}=\frac{1}{h_{r}}\left[\frac{1}{\sigma_{i, j}}\left(\int_{r_{i}}^{r_{i+1 / 2}}\left(2-\frac{r}{r_{i+1 / 2}}\right) d r\right)+\frac{1}{\sigma_{i+1, j}}\left(\int_{r_{i+1 / 2}}^{r_{i+1}}\left(2-\frac{r}{r_{i+1 / 2}}\right) d r\right)\right],
$$


e:

$$
\begin{aligned}
& \int_{r_{i}}^{r_{i+1 / 2}}\left(2-\frac{r}{r_{i+1 / 2}}\right) d r=\frac{h_{r}}{2}\left(2-\frac{r_{i+1 / 4}}{r_{i+1 / 2}}\right)=r_{i}+\frac{3 h_{r}}{4} \\
& \int_{r_{i+1 / 2}}^{r_{i+1}}\left(2-\frac{r}{r_{i+1 / 2}}\right) d r=\frac{h_{r}}{2}\left(2-\frac{r_{i+3 / 4}}{r_{i+1 / 2}}\right)=r_{i}+\frac{h_{r}}{4} .
\end{aligned}
$$

Então:

$$
K_{i+1 / 2, j}^{H}=2 r_{i+1 / 2}\left(\frac{\sigma_{i, j} \sigma_{i+1, j}}{\sigma_{i, j} r_{i+1 / 4}+\sigma_{i+1, j} r_{i+3 / 4}}\right)
$$

e

$$
\begin{aligned}
& a_{i+1 / 2, j}=K_{i+1 / 2, j}^{H} \frac{1}{h_{r}^{2}} \int_{r_{i}}^{r_{i+1}} \frac{r-r_{i+1 / 2}}{\sigma\left(r, \theta_{j}\right)} d r= \\
& 2 r_{i+1 / 2}\left(\frac{\sigma_{i, j} \sigma_{i+1, j}}{\sigma_{i, j} r_{i+1 / 4}+\sigma_{i+1, j} r_{i+3 / 4}}\right) \frac{1}{h_{r}^{2}}\left(\frac{\sigma_{i, j}-\sigma_{i+1, j}}{\sigma_{i, j} \sigma_{i+1, j}}\right),
\end{aligned}
$$

em conseqüência:

$$
a_{i+1 / 2, j}=\frac{r_{i+1 / 2}}{4}\left(\frac{\sigma_{i, j}-\sigma_{i+1, j}}{\sigma_{i, j} r_{i+1 / 4}+\sigma_{i+1, j} r_{i+3 / 4}}\right) \text {. }
$$

O sistema linear na direção radial fica:

Para $i=1,1 \leq j \leq N$ :

$$
r_{3 / 2}\left(W_{1}\right)_{3 / 2, j}=-r_{3 / 2}\left(1+a_{3 / 2, j}\right)^{-1} K_{3 / 2, j}^{H} \frac{u_{2, j}-u_{1, j}}{h_{r}}
$$

Para $2 \leq i \leq M-1,1 \leq j \leq N$ :

$r_{i+1 / 2}\left(W_{1}\right)_{i+1 / 2, j}-r_{i-1 / 2}\left(W_{1}\right)_{i-1 / 2, j}=-\left(1+a_{i+1 / 2, j}-a_{i-1 / 2, j}\right)^{-1}\left[r_{i+1 / 2} K_{i+1 / 2, j}^{H} \frac{u_{i+1, j}-u_{i, j}}{h_{r}}-r_{i-1 / 2} K_{i-1 / 2, j}^{H} \frac{u_{i, j}-u_{i-1, j}}{h_{r}}\right]$

Para $i=M, 1 \leq j \leq N$ :

$r_{M+1 / 2}\left(W_{1}\right)_{M+1 / 2, j}-r_{M-1 / 2}\left(W_{1}\right)_{M-1 / 2, j}=-\left(1+a_{M+1 / 2, j}-a_{M-1 / 2, j}\right)^{-1}\left[r_{M+1 / 2} K_{M+1 / 2, j}^{H} \frac{g_{j}-u_{M, j}}{h_{r}}-r_{M-1 / 2} K_{M-1 / 2, j}^{H} \frac{u_{M, j}-u_{M-1, j}}{h_{r}}\right]$ 


\section{Na direção angular:}

Para $1 \leq j \leq N, \quad 1 \leq i \leq M$

$$
-\left(1+a_{i, j+1 / 2}-a_{i, j-1 / 2}\right)^{-1}\left[K_{i, j+1 / 2}^{H} \frac{u_{i, j+1}-u_{i, j}}{r_{i} h_{\theta}}-K_{i, j-1 / 2}^{H} \frac{u_{i, j}-u_{i, j-1}}{r_{i} h_{\theta}}\right]=\left(W_{2}\right)_{i, j+1 / 2}-\left(W_{2}\right)_{i, j-1 / 2}
$$

\section{Aproximação nas interfaces:}

Nos intervalos $\left[\theta_{j}, \theta_{j+1}\right]$, os valores de $K_{i+1 / 2, j}^{H}, a_{i+1 / 2, j}$ podem ser aproximados pelo método do ponto médio, então:

$$
\begin{gathered}
K_{i, j+1 / 2}^{H}=2\left(\frac{\sigma_{i, j} \sigma_{i, j+1}}{\sigma_{i, j}+\sigma_{i, j+1}}\right) \\
a_{i, j+1 / 2}=K_{i, j+1 / 2}^{H} \frac{1}{h_{\theta}^{2}} \int_{\theta_{j}}^{\theta_{j+1}} \frac{\theta-\theta_{j+1 / 2}}{\sigma\left(r_{i}, \theta\right)} d \theta=2\left(\frac{\sigma_{i, j} \sigma_{i, j+1}}{\sigma_{i, j}+\sigma_{i, j+1}}\right) \frac{1}{8}\left(\frac{\sigma_{i, j}-\sigma_{i, j+1}}{\sigma_{i, j} \sigma_{i, j+1}}\right),
\end{gathered}
$$

então:

$$
a_{i, j+1 / 2}=\frac{1}{4}\left(\frac{\sigma_{i, j}-\sigma_{i, j+1}}{\sigma_{i, j}+\sigma_{i, j+1}}\right)
$$

Em $j=1$, temos que levar em consideração os termos:

$$
\begin{gathered}
K_{i, 1-1 / 2}^{H}=2\left(\frac{\sigma_{i, 1} \sigma_{i, N}}{\sigma_{i, 1}+\sigma_{i, N}}\right), \\
a_{i, 1-1 / 2}=\frac{1}{4}\left(\frac{\sigma_{i, 1}-\sigma_{i, N}}{\sigma_{i, 1}+\sigma_{i, N}}\right),
\end{gathered}
$$

então temos que na direção angular o sistema linear fica:

Para $1 \leq j \leq N, 1 \leq i \leq M$ :

$$
\left(W_{2}\right)_{i, j+1 / 2}-\left(W_{2}\right)_{i, j-1 / 2}=-\left(1+a_{i, j+1 / 2}-a_{i, j-1 / 2}\right)^{-1}\left[K_{i, j+1 / 2}^{H} \frac{u_{i, j+1}-u_{i, j}}{r_{i} h_{\theta}}-K_{i, j-1 / 2}^{H} \frac{u_{i, j}-u_{i, j-1}}{r_{i} h_{\theta}}\right]
$$


Detalhamento dos termos $K_{i+1 / 2, j}^{H}, K_{i, j+1 / 2}^{H}$

Suponha que a interface se encontra nos pontos médios de $\left[\theta_{N / 4}, \theta_{N / 4+1}\right]$ e $\left[\theta_{N / 2}, \theta_{N / 2+1}\right]$ então:

$$
K_{i+1 / 2, j}^{H}=\left\{\begin{array}{l}
\sigma_{i, j}, i=1, M / 2-1, j=1, N \\
2 r_{i+1 / 2}\left(\frac{\sigma_{i, j} \sigma_{i+1, j}}{\sigma_{i, j} r_{i+1 / 4}+\sigma_{i+1, j} r_{i+3 / 4}}\right), i=M / 2, j=1, N \\
\sigma_{i, j}, i=M / 2+1, M, j=1, N
\end{array}\right.
$$

e

$$
K_{i, j+1 / 2}^{H}=\left\{\begin{array}{l}
\sigma_{i, j}, \quad j=1, N / 4-1, i=1, M \\
2\left(\frac{\sigma_{i, j} \sigma_{i, j+1}}{\sigma_{i, j}+\sigma_{i, j+1}}\right), j=N / 4, \quad i=1, M \\
\sigma_{i, j}, \quad j=N / 4+1, N / 2-1, \quad i=1, M \\
2\left(\frac{\sigma_{i, j} \sigma_{i, j+1}}{\sigma_{i, j}+\sigma_{i, j+1}}\right), j=N / 2, i=1, M \\
\sigma_{i, j}, j=N / 2+1, N-1, i=1, M \\
2\left(\frac{\sigma_{i, N} \sigma_{i, 1}}{\sigma_{i, 1}+\sigma_{i, N}}\right), j=N, \quad i=1, M
\end{array}\right.
$$

Montagem da Matriz $A G$ de $(A G) U=b$

Substituindo (4.86) e (4.94) em (4.42) e considerando $A(i, j), B(i, j), C(i, j)$ como sendo os coeficientes das variáveis $u_{i j}$ em (4.42), montamos o sistema linear $(A G) * U=b$ correspondente ao esquema HIA.

Para $i=1, \quad j=1, N$

$$
\left\{\begin{array}{l}
C(1,1) u_{1,1}-B S(1,1) u_{1,2}-B(1,1) u_{1, N}-A(1,1) u_{2,1}=r_{1} f_{1,1} \\
-B F(i, j-1) u_{i, j-1}+C(1, j) u_{1, j}-B S(1, j) u_{1, j+1}-A(1, j) u_{2, j}=r_{1} f_{1, j}, j=2, N-1 . \\
-B(1, N) u_{1,1}-B F(1, N-1) u_{1, N-1}+C(1, N) u_{1, N}-A(1, N) u_{2, N}=r_{1} f_{1, N}
\end{array}\right.
$$

Assim temos os primeiros blocos de $A G$ ficam: 


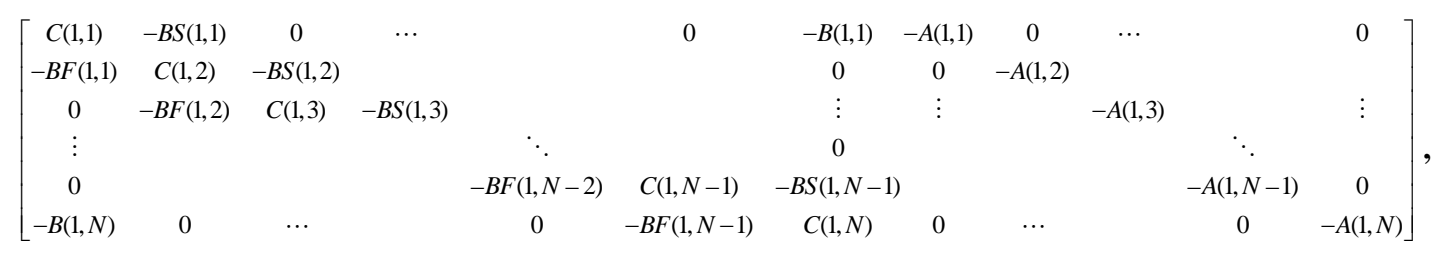

onde $B(1, N) \neq B(1,1)$.

Para $i=2, M-1 \quad j=1, N$

$$
\left\{\begin{array}{l}
-A(i-1,1) u_{i-1,1}+C(i, 1) u_{i, 1}-B S(i, 1) u_{i, 2}-B(i, 1) u_{i, N}-A(i, 1) u_{i+1,1}=r_{i} f_{i, 1} \\
-A(i-1, j) u_{i-1, j}-B F(i, j-1) u_{i, j-1}+C(i, j) u_{i, j}-B S(i, j) u_{i, j+1}-A(i, j) u_{i+1, j}=r_{i} f_{i, j}, j=2, N-1 \\
-A(i-1, N) u_{i-1, N}-B(i, N) u_{i, 1}-B F(i, N-1) u_{i, N-1}+C(i, N) u_{i, N}-A(i, N) u_{i+1, N}=r_{i} f_{i, N}
\end{array}\right.
$$

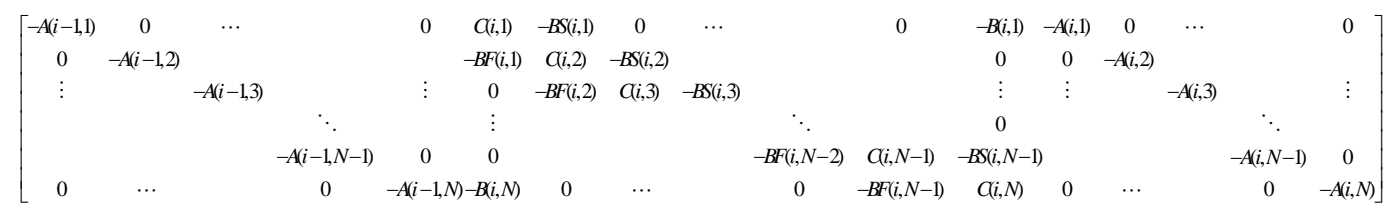

onde $B(i, N) \neq B(i, 1)$

Para $i=M \quad j=1, N$

$$
\left\{\begin{array}{l}
-A(M-1,1) u_{M-1,1}+C(M, 1) u_{M, 1}-B S(M, 1) u_{M, 2}-B(M, 1) u_{M, N}=r_{M} f_{M, 1}+A(M, 1) g(1) \\
-A(M-1, j) u_{M-1, j}-B F(M, j-1) u_{M, j-1}+C(M, j) u_{M, j}-B S(M, j) u_{M, j+1}=r_{M} f_{M, j}+A(M, j) g(j), j=2, N-1 \\
-A(M-1, N) u_{M-1, N}-B(M, N) u_{M, 1}-B F(M, N-1) u_{M, N-1}+C(M, N) u_{M, N}=r_{1} f_{1, N}+A(M, N) g(N)
\end{array},\right.
$$

então os últimos blocos de $A G$ são:

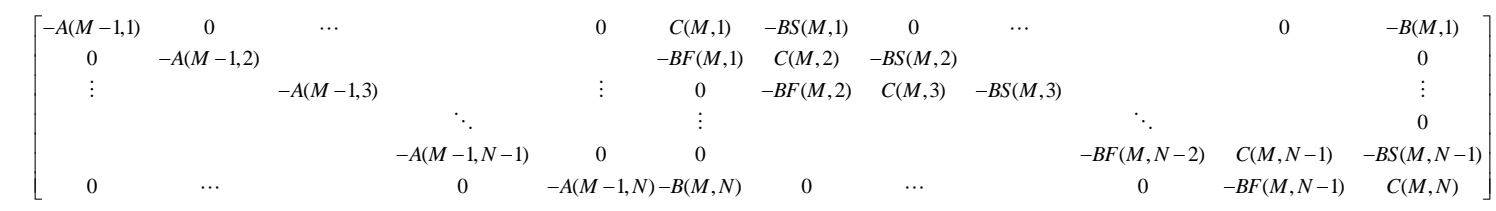

$B(M, N) \neq B(M, 1)$

Portanto, a matriz $A G$ (em blocos) fica da seguinte forma: 


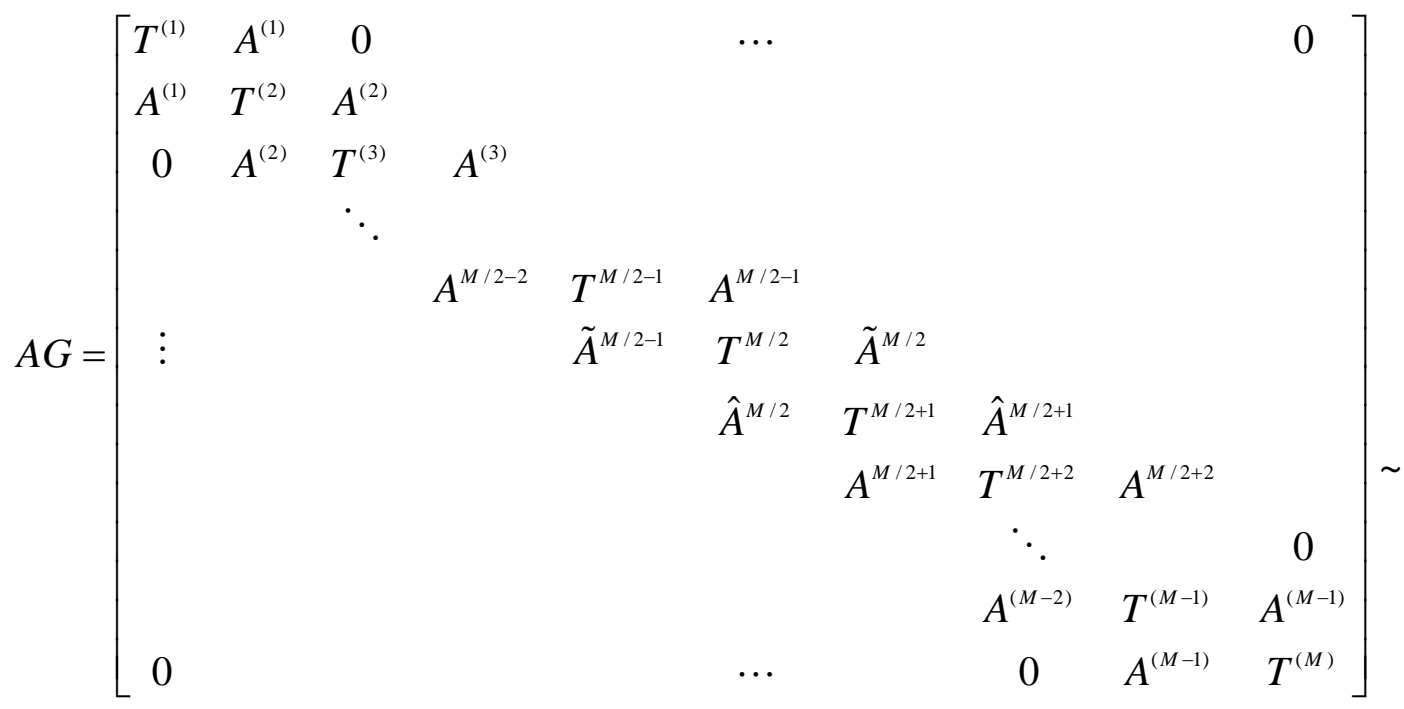

onde: $\tilde{A}^{(M / 2-1)} \neq A^{(M / 2-1)}, \quad \tilde{A}^{(M / 2)} \neq \hat{A}^{(M / 2)}, \quad \hat{A}^{(M / 2+1)} \neq A^{(M / 2+1)}$. A matriz $A G$ é não simétrica.

\section{Resultados}

Consideramos os seguintes problemas:

\section{Problema (5)}

$$
\begin{aligned}
& u(r, \theta)=\left(r^{2}-r_{m}\right)^{2} / 4 \sigma(r, \theta) \\
& \sigma(r, \theta)=\left\{\begin{array}{lll}
\sigma_{1}, & 0<r<r_{m}, & 0 \leq \theta \leq 2 \pi \\
\sigma_{2}, & r_{m} \leq r \leq 1, & 0 \leq \theta \leq 2 \pi
\end{array}\right.
\end{aligned}
$$

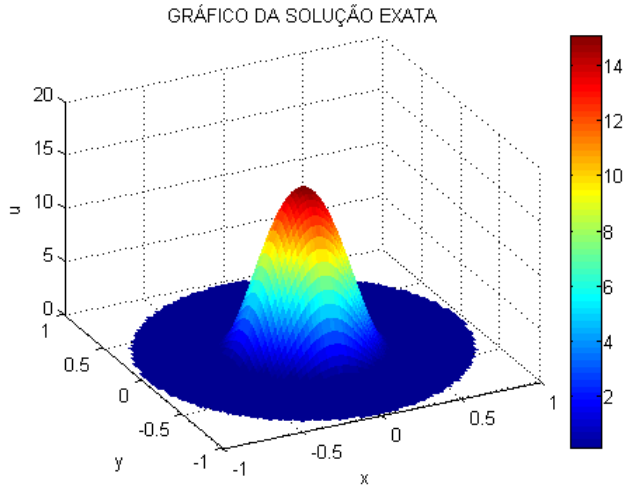

(a)

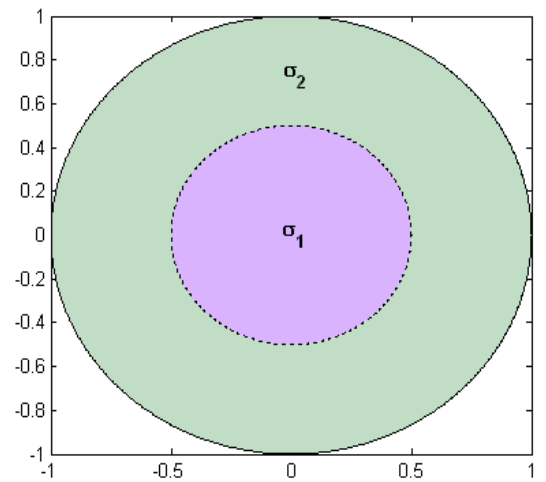

(b)

Figura 4.16 (a) Gráfico da solução exata do Problema (5), ( b) Interface no disco unitário. 
A interface $\Gamma$ é:

$$
\begin{gathered}
\Gamma=\left\{(r, \theta) / r=r_{m} ; \quad 0 \leq \theta \leq 2 \pi\right\} \\
5.1-\sigma(r, \theta)=\left\{\begin{array}{ll}
1, & 0<r<r_{m}, 0 \leq \theta \leq 2 \pi \\
100, & r_{m} \leq r \leq 1,0 \leq \theta \leq 2 \pi
\end{array},\right.
\end{gathered}
$$

Considerando a interface em $r_{m}=r_{M / 2}+0.5 h_{r}$ e usando a condição de fronteira tipo Dirichlet, obtemos os seguintes resultados:

Tabela 4.21 Aproximação do problema 5.1 usando o método HA e fatorização de Choleski para resolver o sistema linear.

\begin{tabular}{|c|c|}
\hline $\mathbf{M}$ & $\begin{array}{c}\text { Fatorização Choleski } \\
r_{m}=r_{M / 2}+0.5 h_{r}\end{array}$ \\
\hline & $\begin{array}{c}\text { Erro relativo na } \\
\text { norma } L^{2}\end{array}$ \\
\hline $\mathbf{1 6}$ & 0.0070624 \\
\hline $\mathbf{3 2}$ & 0.00154876 \\
\hline $\mathbf{6 4}$ & $3.7760161 \times 10^{-4}$ \\
\hline $\mathbf{1 2 8}$ & $9.4292309 \times 10^{-5}$ \\
\hline
\end{tabular}

Como podemos observar na tabela 4.21, o esquema HA tem convergência quadrática o qual era o esperado pela ordem do esquema usado.

Usando a condição de fronteira tipo Neumann e aplicando fatorização de Choleski para resolver o sistema linear obtemos:

Tabela 4.22 Aproximação do problema 5.1 com condição de fronteira tipo Neumann usando o método HA e fatorização de Choleski para resolver o sistema linear.

\begin{tabular}{|c|c|}
\hline $\mathbf{M}$ & $\begin{array}{c}\text { Fatorização de Choleski } \\
r_{m}=r_{M / 2}+0.5 h_{r}\end{array}$ \\
\hline & $\begin{array}{c}\text { Erro relativo na norma } \\
L^{2}\end{array}$ \\
\hline $\mathbf{1 6}$ & 0.0251498 \\
\hline $\mathbf{3 2}$ & 0.0062746 \\
\hline $\mathbf{6 4}$ & 0.0015699 \\
\hline $\mathbf{1 2 8}$ & $3.9282675 \times 10^{-4}$ \\
\hline
\end{tabular}


Considerando a interface em $r=0.5$ e usando condição de fronteira tipo Dirichlet:

Tabela 4.23 Aproximação do problema 5.1, onde a interface se encontra em 0.5 usando os métodos HA, HIA e eliminação gaussiana para resolver o sistema linear.

\begin{tabular}{|c|c|c|}
\hline \multicolumn{3}{|c|}{ Eliminação Gaussiana } \\
$r=0.5$ \\
\hline GRID & HA & HIA \\
\hline $\mathbf{M}$ & $\begin{array}{c}\text { Erro relativo na } \\
\text { norma } L^{2}\end{array}$ & $\begin{array}{c}\text { Erro relativo na } \\
\text { norma } L^{2}\end{array}$ \\
\hline $\mathbf{1 6}$ & 0.00706241 & 0.0091825 \\
\hline $\mathbf{3 2}$ & 0.0015488 & 0.0026156 \\
\hline $\mathbf{6 4}$ & $3.7760161 \times 10^{-4}$ & $7.0247739 \times 10^{-4}$ \\
\hline $\mathbf{1 2 8}$ & $9.4292309 \times 10^{-5}$ & $1.8230732 \times 10^{-4}$ \\
\hline
\end{tabular}

Observamos da tabela 4.23 que os esquemas HA e HIA têm convergência quadrática quando consideramos condição de fronteira tipo Dirichlet. Porém, os resultados do esquema HIA não têm a precisão obtida em coordenadas cartesianas.

$$
\text { 5.2- } \sigma(r, \theta)= \begin{cases}0.001, & 0<r<r_{m}, \quad 0 \leq \theta \leq 2 \pi \\ 1000, & r_{m} \leq r \leq 1, \quad 0 \leq \theta \leq 2 \pi\end{cases}
$$

Considerando a interface em $r_{m}=r_{M / 2}+0.5 h_{r}$ e usando à condição de Dirichlet:

Tabela 4.24 Aproximação do problema 5.2 usando o método HA e fatorização de Choleski para resolver o sistema linear.

\begin{tabular}{|c|c|}
\hline $\mathbf{M}$ & $\begin{array}{c}\text { Fatorização de Choleski } \\
r_{m}=r_{M / 2}+0.5 h_{r}\end{array}$ \\
\hline & $\begin{array}{c}\text { Erro relativo na norma } \\
L^{2}\end{array}$ \\
\hline $\mathbf{1 6}$ & 0.0066151 \\
\hline $\mathbf{3 2}$ & 0.0016981 \\
\hline $\mathbf{6 4}$ & $4.2897791 \times 10^{-4}$ \\
\hline $\mathbf{1 2 8}$ & $1.0773579 \times 10^{-4}$ \\
\hline
\end{tabular}


Usando à condição de Neumann temos os seguintes resultados:

Tabela 4.25 Aproximação do problema 5.2 com condição de fronteira tipo Neumann usando o método HA e fatoirização de Choleski para resolver o sistema linear.

\begin{tabular}{|c|c|}
\hline $\mathbf{M}$ & $\begin{array}{c}\text { Fatorização de Choleski } \\
r_{m}=r_{M / 2}+0.5 h_{r}\end{array}$ \\
\hline & Erro relativo na norma \\
& $L^{2}$ \\
\hline $\mathbf{1 6}$ & 0.0232906 \\
\hline $\mathbf{3 2}$ & 0.0057873 \\
\hline $\mathbf{6 4}$ & 0.0014446 \\
\hline $\mathbf{1 2 8}$ & $3.6101586 \times 10^{-4}$ \\
\hline
\end{tabular}

Nas tabelas 4.24 e 4.25 observamos que o esquema HA tem convergência quadrática para saltos grandes nas interfaces.

Considerando a interface em $r=0.5$, temos:

Tabela 4.26 Aproximação do problema 5.2, onde a interface se encontra em 0.5 usando os métodos HA, HIA e eliminação gaussiana para resolver o sistema linear.

\begin{tabular}{|c|c|c|c|c|}
\hline \multicolumn{5}{|c|}{$\begin{array}{c}\text { Eliminação Gaussiana } \\
r=0.5\end{array}$} \\
\hline GRID & \multicolumn{2}{|c|}{ HA } & \multicolumn{2}{c|}{ HIA } \\
\hline $\mathbf{M}$ & $\begin{array}{c}\text { Erro relativo na } \\
\text { norma } L^{2}\end{array}$ & Tempo & $\begin{array}{c}\text { Erro relativo na } \\
\text { norma } L^{2}\end{array}$ & Tempo \\
\hline $\mathbf{1 6}$ & 0.0065915 & 0.09375 & 0.0089974 & 0.125 \\
\hline $\mathbf{3 2}$ & 0.0014729 & 0.234375 & 0.0025751 & 0.265625 \\
\hline $\mathbf{6 4}$ & $3.6553819 \times 10^{-4}$ & 1.8125 & $6.9321404 \times 10^{-4}$ & 1.4375 \\
\hline $\mathbf{1 2 8}$ & $9.2187789 \times 10^{-5}$ & 22.09375 & $1.8011226 \times 10^{-4}$ & 13.78125 \\
\hline
\end{tabular}

Na tabela 4.26 observamos que os esquemas HA e HIA têm convergência quadrática. Porém, o esquema HIA não têm a precisão obtida em coordenadas cartesianas. 


\section{Problema (6)}

$$
u(r, \theta)=r^{3}\left(r-r_{m}\right)^{3} \sin (z \theta)\left(\cos ^{2}(z \theta)-1\right) / \sigma(r, \theta), \text { o coeficiente } z \text { é tal que } z \theta=\pi
$$

$$
\sigma(r, \theta)= \begin{cases}\sigma_{1}, & 0<r<r_{m}, \quad 0 \leq \theta<y_{n} \\ \sigma_{2}, & 0<r<r_{m}, \quad y_{n}<\theta \leq 2 \pi \\ \sigma_{3}, & r_{m}<r \leq 1, \quad 0 \leq \theta \leq 2 \pi\end{cases}
$$

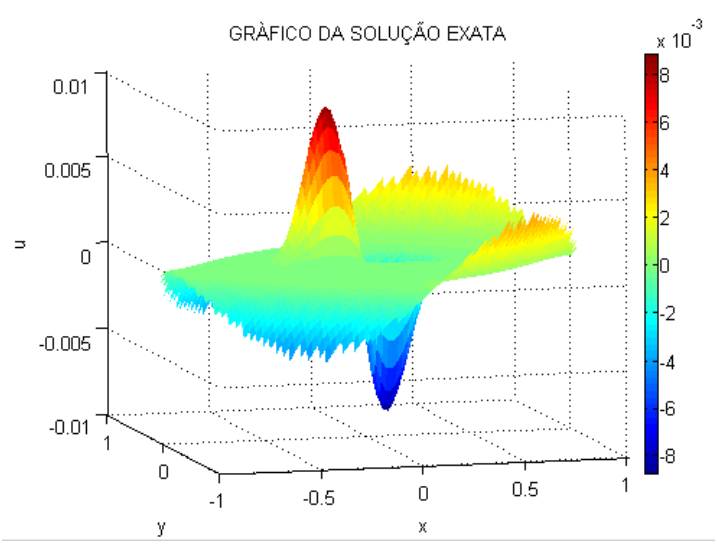

(a)

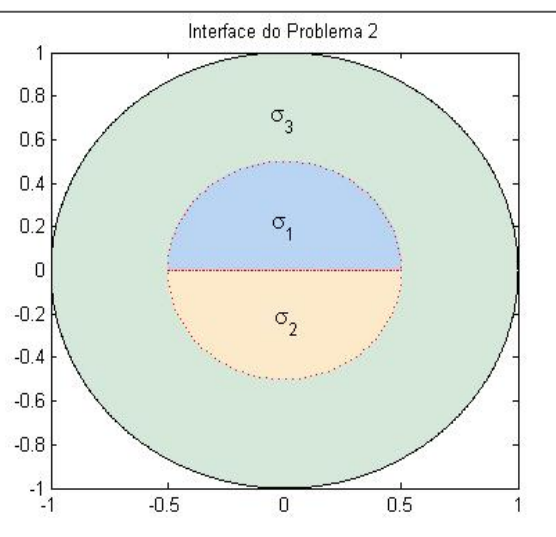

(b)

Figura 4.17 (a) Gráfico da solução exata do Problema (6), (b) Interfaces no disco unitário.

A interface $\Gamma \subset \Omega$ tem a forma $\Gamma=\Gamma_{1} \cup \Gamma_{2} \cup \Gamma_{3}$, onde:

$$
\left\{\begin{array}{ll}
\Gamma_{1}=\left\{(r, \theta) / r=r_{M / 2}+\frac{h_{r}}{2}, \quad 0 \leq \theta \leq 2 \pi\right\} \\
\Gamma_{2}=\left\{(r, \theta) / \theta=\frac{\pi}{4},\right. & \left.0<r \leq r_{m}\right\} \\
\Gamma_{3}=\left\{(r, \theta) / \theta=\frac{\pi}{2},\right. & \left.0<r \leq r_{m}\right\}
\end{array},\right.
$$

a qual divide o disco unitário em 3 partes.

Interface no ponto médio do intervalo central para cada um dos eixos

$$
\text { 6.1- } \sigma(r, \theta)=\left\{\begin{array}{l}
\sigma_{1}=1 \\
\sigma_{2}=10 \\
\sigma_{3}=100
\end{array}\right.
$$


Aplicando a condição de fronteira tipo Dirichlet e sem considerar o pólo:

Tabela 4.27 Aproximações do problema 6.1, usando os métodos HA , HIA e eliminação gaussiana para resolver o sistema linear.

\begin{tabular}{|c|c|c|}
\hline \multicolumn{3}{|c|}{$\begin{array}{c}\text { Eliminação Gaussiana } \\
r_{m}=r_{M / 2}+0.5 h_{r} \\
y_{n}=\theta_{N / 2}+0.5 h_{\theta}\end{array}$} \\
\hline GRID & HA & HIA \\
\hline $\mathbf{M}$ & $\begin{array}{c}\text { Erro relativo na } \\
\text { norma } L^{2}\end{array}$ & $\begin{array}{c}\text { Erro relativo na } \\
\text { norma } L^{2}\end{array}$ \\
\hline 16 & 0.0131015 & 0.0122549 \\
\hline 32 & 0.0025788 & 0.0024479 \\
\hline 64 & $5.743182 \times 10^{-4}$ & $5.4833134 \times 10^{-4}$ \\
\hline 128 & $1.3660386 \times 10^{-4}$ & $1.3056715 \times 10^{-4}$ \\
\hline
\end{tabular}

Interface no ponto médio do intervalo central para cada um dos eixos

$$
\text { 6.2- } \sigma(r, \theta)=\left\{\begin{array}{l}
\sigma_{1}=1 \\
\sigma_{2}=10 \\
\sigma_{3}=0.001
\end{array}\right.
$$

Aplicando à condição de fronteira tipo Dirichlet e sem considerar o pólo:

Tabela 4.28 Aproximações do problema 6.2, usando os métodos HA , HIA e eliminação gaussiana para resolver o sistema linear.

\begin{tabular}{|c|c|c|}
\hline \multicolumn{3}{|c|}{$\begin{array}{c}\text { Eliminação Gaussiana } \\
r_{m}=r_{M / 2}+0.5 h_{r}\end{array}$} \\
& \multicolumn{2}{|c|}{$y_{n}=\theta_{N / 2}+0.5 h_{\theta}$} \\
\hline GRID & HA & HIA \\
\hline $\mathbf{M}$ & $\begin{array}{c}\text { Erro relativo na } \\
\text { norma } L^{2}\end{array}$ & $\begin{array}{c}\text { Erro relativo na } \\
\text { norma } L^{2}\end{array}$ \\
\hline $\mathbf{1 6}$ & 0.0126043 & 0.0081841 \\
\hline $\mathbf{3 2}$ & 0.0023572 & 0.0015995 \\
\hline $\mathbf{6 4}$ & $4.7971441 \times 10^{-4}$ & $3.5345334 \times 10^{-4}$ \\
\hline $\mathbf{1 2 8}$ & $1.0502971 \times 10^{-4}$ & $8.3291517 \times 10^{-5}$ \\
\hline
\end{tabular}

Das tabelas 4.27 e 4.28 podemos ver que o esquema HIA aproximou um pouco melhor o problema. Porém os resultados não são tão satisfatórios quando os obtidos em coordenadas cartesianas. 


\subsection{Aproximação da solução na fronteira}

Para aproximar a solução na fronteira usamos os polinômios interpoladores de Lagrange e os três pontos finais na direção radial, como mostra a figura 4.18:

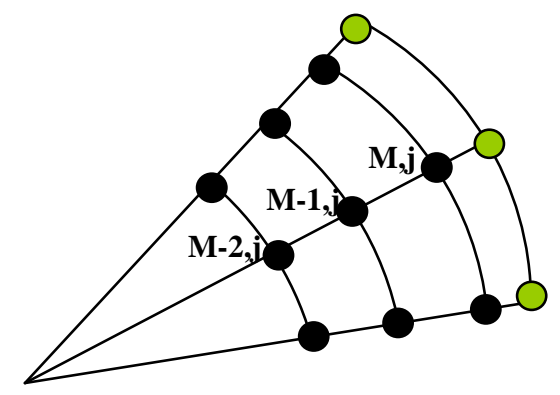

Figura 4.18 Gráfico da extrapolação na fronteira.

Os polinômios de Lagrange na direção radial são:

$$
\left\{\begin{array}{l}
L_{M-2}(r)=\frac{\left(r-r_{M-1}\right)\left(r-r_{M}\right)}{\left(r_{M-2}-r_{M-1}\right)\left(r_{M-2}-r_{M}\right)} \\
L_{M-1}(r)=\frac{\left(r-r_{M-2}\right)\left(r-r_{M}\right)}{\left(r_{M-1}-r_{M-2}\right)\left(r_{M-1}-r_{M}\right)} \\
L_{M}(r)=\frac{\left(r-r_{M-2}\right)\left(r-r_{M-1}\right)}{\left(r_{M}-r_{M-2}\right)\left(r_{M}-r_{M-1}\right)}
\end{array}\right.
$$

Assim, os valores desses polinômios em $r=1.0$, considerando condição de fronteira tipo Neumann são:

$$
L_{M-2}(1)=\frac{3}{8}, L_{M-1}(1)=\frac{-5}{4}, L_{M}(1)=\frac{15}{8} .
$$

Portanto, os valores extrapolados na fronteira têm a forma:

$$
u\left(1, \theta_{j}\right) \approx \sum_{k=0}^{2} L_{M-k}(1) u_{M-k, j}
$$




\section{Resultados da extrapolação}

Consideramos os problema 1, 2, 4 e 5.2, com condição de fronteira tipo Neumann e comparamos a solução exata em $r=1$ e a aproximação usando valores extrapolados seguindo a formula 4.103 .

Tabela 4.29 Extrapolação na fronteira do problema (1)

\begin{tabular}{|c|c|}
\hline $\mathbf{M}$ & $\begin{array}{c}\text { Problema (1) } \\
\text { PGC - Choleski Incompleto }\end{array}$ \\
\hline & Erro máximo \\
\hline $\mathbf{1 6}$ & 0.0123518 \\
\hline $\mathbf{3 2}$ & 0.0031503 \\
\hline $\mathbf{6 4}$ & $7.9901749 \times 10^{-4}$ \\
\hline $\mathbf{1 2 8}$ & $2.0107814 \times 10^{-4}$ \\
\hline
\end{tabular}

Tabela 4.30 Extrapolação na fronteira do problema (2)

\begin{tabular}{|c|c|}
\hline $\mathbf{M}$ & $\begin{array}{c}\text { Problema (2) } \\
\text { PGC - Choleski Incompleto }\end{array}$ \\
\hline & Erro máximo \\
\hline $\mathbf{1 6}$ & 0.0032864 \\
\hline $\mathbf{3 2}$ & $8.0221371 \times 10^{-4}$ \\
\hline $\mathbf{6 4}$ & $1.9851531 \times 10^{-4}$ \\
\hline $\mathbf{1 2 8}$ & $4.9463472 \times 10^{-5}$ \\
\hline
\end{tabular}

Tabela 4.31 Extrapolação na fronteira do problema (4) usando o método HIA.

\begin{tabular}{|c|c|}
\hline $\mathbf{N}=\mathbf{M}$ & $\begin{array}{c}\text { Problema (4) } \\
\text { PGC - Choleski Incompleto }\end{array}$ \\
\hline & Erro máximo \\
\hline $\mathbf{1 2}$ & 0.0905839 \\
\hline $\mathbf{2 2}$ & 0.0179451 \\
\hline $\mathbf{4 2}$ & 0.0069149 \\
\hline $\mathbf{8 2}$ & 0.0023355 \\
\hline $\mathbf{1 6 2}$ & $5.6768302 \times 10^{-4}$ \\
\hline
\end{tabular}


Tabela 4.32 Extrapolação na fronteira do problema 5.2, usando o método HIA.

\begin{tabular}{|c|c|}
\hline $\mathbf{M}$ & $\begin{array}{c}\text { Eliminação Gaussiana } \\
r_{m}=r_{M / 2}+0.5 h_{r}\end{array}$ \\
\hline & Erro máximo \\
\hline $\mathbf{1 6}$ & $5.2309479 \times 10^{-6}$ \\
\hline $\mathbf{3 2}$ & $2.3690984 \times 10^{-6}$ \\
\hline $\mathbf{6 4}$ & $9.2975629 \times 10^{-6}$ \\
\hline $\mathbf{1 2 8}$ & $8.1822563 \times 10^{-8}$ \\
\hline
\end{tabular}

Dos resultados das tabelas 4.29, 4.30, 4.31 e 4.32 podemos concluir que o uso dos polinômios interpeladores de Lagrange resulta em aproximações razoáveis na fronteira. No entanto outros métodos, como por exemplo, splines cúbicos podem ser usados para tal fim. 


\section{ESTUDOS NUMÉRICOS NA TIE}

Neste capitulo, vamos aplicar os métodos de solução numérica do problema direto descritos no capitulo 4 e também consideramos uma linearização para resolver o problema inverso.

\subsection{A Malha}

Consideramos o domínio condutor como sendo o disco unitário.

Consideramos $M$ subdivisões no eixo radial e $N=2 M$ subdivisões no eixo angular. Assim temos:

Número de nós $=M * N$ nós no interior do disco e $N$ nós na fronteira.

Número de volumes finitos $=M * N$.

Número de eletrodos alocados na fronteira $=16$.

MALHA: $M=16, N=32$

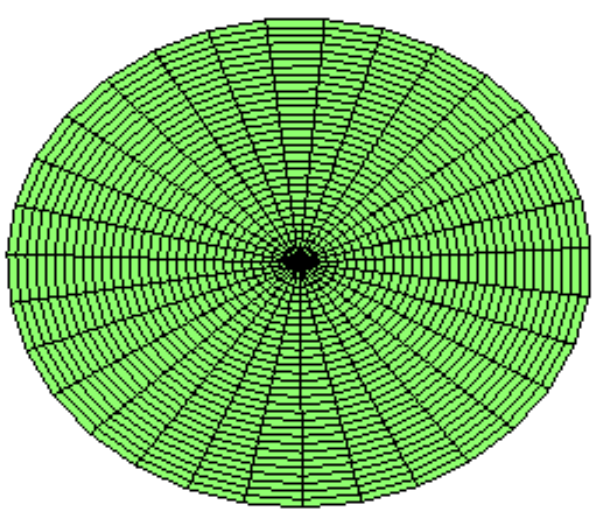

(a)

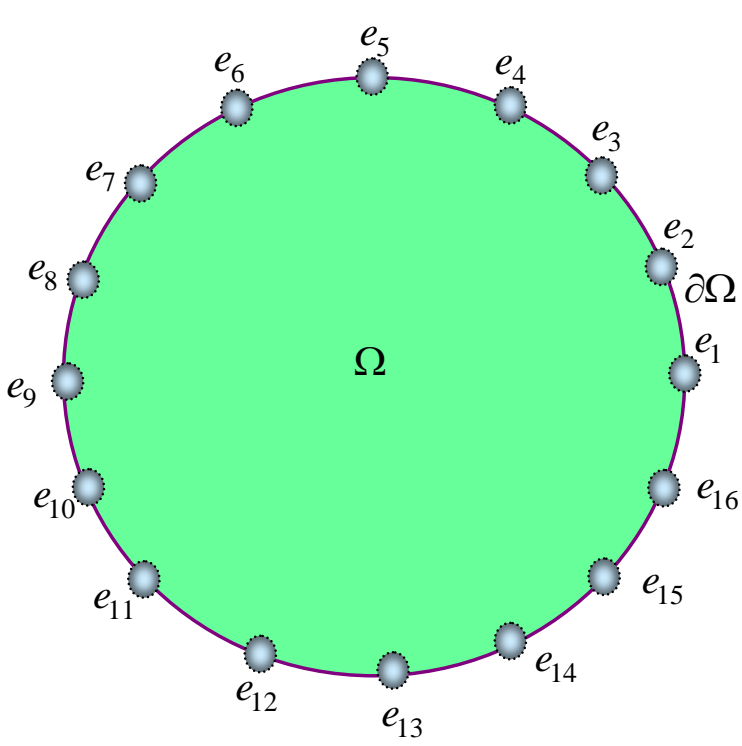

(b)

Figura 5.1 (a) Malha com volumes finitos no disco unitário quando usamos 16 subdivisões no eixo radial e 32 subdivisões no eixo angular, (b) Posição dos 16 eletrodos na fronteira. 


\subsection{O Problema Direto}

Para termos uma aproximação dos valores dos potenciais nos eletrodos, consideramos a equação de difusão (2.13) em coordenadas polares:

$$
\frac{-1}{r}\left[\frac{\partial}{\partial r}\left(\sigma(r, \theta) r \frac{\partial u}{\partial r}\right)+\frac{\partial}{\partial \theta}\left(\frac{\sigma(r, \theta)}{r} \frac{\partial u}{\partial \theta}\right)\right]=0, \quad \text { em } \Omega,
$$

junto às condições de fronteira (2.19):

$$
I^{d}=\left\{\begin{array}{l}
\frac{I_{l}}{\left|e_{l}\right|}, x \in e_{l}, l=1,2, . ., L \\
0, x \in \partial \Omega \backslash \bigcup_{l=1}^{L} e_{l}
\end{array},\right.
$$

onde consideramos $d=1,2,3, . ., P$ padrões de corrente injetados nos $l=1,2, \ldots, L=16$ eletrodos.

Os padrões de correntes injetadas nos 16 eletrodos correspondem aos modelos: adjacente e pula 1. Os padrões de corrente do tipo adjacente são da forma:

$$
I^{1}=\left[\begin{array}{c}
-I \\
I \\
0 \\
0 \\
\vdots \\
0 \\
0 \\
0
\end{array}\right], I^{2}=\left[\begin{array}{c}
0 \\
-I \\
I \\
0 \\
\vdots \\
0 \\
0 \\
0
\end{array}\right], I^{3}=\left[\begin{array}{c}
0 \\
0 \\
-I \\
I \\
0 \\
\vdots \\
0 \\
0
\end{array}\right], \ldots, I^{15}=\left[\begin{array}{c}
0 \\
0 \\
0 \\
0 \\
0 \\
\vdots \\
-I \\
I
\end{array}\right], I^{16}=\left[\begin{array}{c}
I \\
0 \\
0 \\
0 \\
0 \\
\vdots \\
0 \\
-I
\end{array}\right],
$$

e os padrões que correspondem á injeção pula 1 são : 


$$
I^{1}=\left[\begin{array}{c}
-I \\
0 \\
I \\
0 \\
\vdots \\
0 \\
0 \\
0
\end{array}\right], I^{2}=\left[\begin{array}{c}
0 \\
-I \\
0 \\
I \\
\vdots \\
0 \\
0 \\
0
\end{array}\right], I^{3}=\left[\begin{array}{c}
0 \\
0 \\
-I \\
0 \\
I \\
\vdots \\
0 \\
0
\end{array}\right], \ldots, I^{15}=\left[\begin{array}{c}
I \\
0 \\
0 \\
0 \\
\vdots \\
0 \\
-I \\
0
\end{array}\right], I^{16}=\left[\begin{array}{c}
0 \\
I \\
0 \\
0 \\
0 \\
\vdots \\
0 \\
-I
\end{array}\right],
$$

onde $I^{d} \in \mathfrak{R}^{L}$.

Resolvemos o problema direto usando o método dos volumes finitos do tipo HA descrito no capitulo anterior. As variáveis são os valores do potencial elétrico no interior e na fronteira do disco unitário. Assim temos $(M+1) N$ variáveis.

O sistema linear tem a forma:

$$
(A G) U=L D
$$

ou

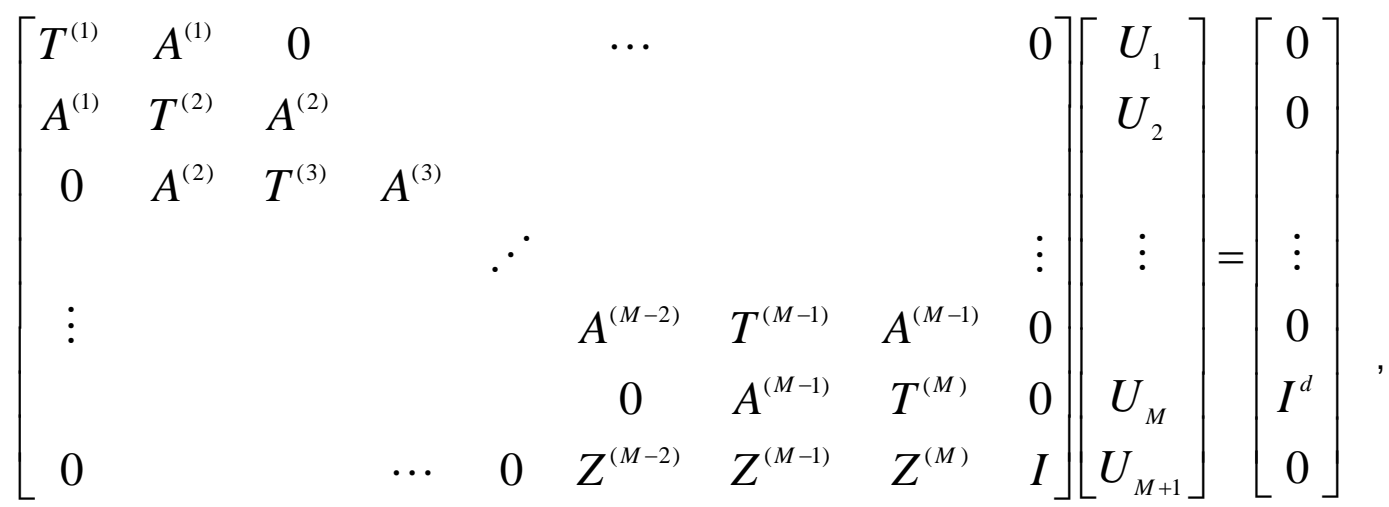

onde $A G$ é uma $M$ - matriz quadrada, não simétrica de dimensão $(M+1) N^{2}, I^{d}$ contem os valores das correntes injetadas nos $L$ eletrodos em $d=1,2,3, . ., P$ padrões. A última linha das matrizes em bloco de $A G$ correspondem á interpolação feita para aproximar as voltagens na fronteira do disco, os vetores $U_{i}$ são vetores que correspondem às $N$ variáveis para cada subdivisão $i$ do eixo radial, $U_{i} \in R^{N}$. 
Para resolver o sistema (5.1) é preciso usar alguma técnica de normalização, dado que a solução desse sistema não é única. No apêndice A podemos encontrar algumas técnicas de regularização, neste caso particular, usamos regularização pela fronteira.

Por outro lado, segundo [67] podemos considerar a voltagem de um nó de referencia igual a zero (voltagem de referencia ou terra). Como conseqüência de usar este critério eliminou-se a indeterminação do sistema linear (5.1).

\subsection{Comparações das voltagens}

A seguir, aproximamos as voltagens nos eletrodos correspondentes à injeção de correntes do tipo adjacente e pula 1. Para resolver o sistema linear usamos normalização pela fronteira e também o método que leva em conta um nó de referencia.

\section{ADJACENTE}

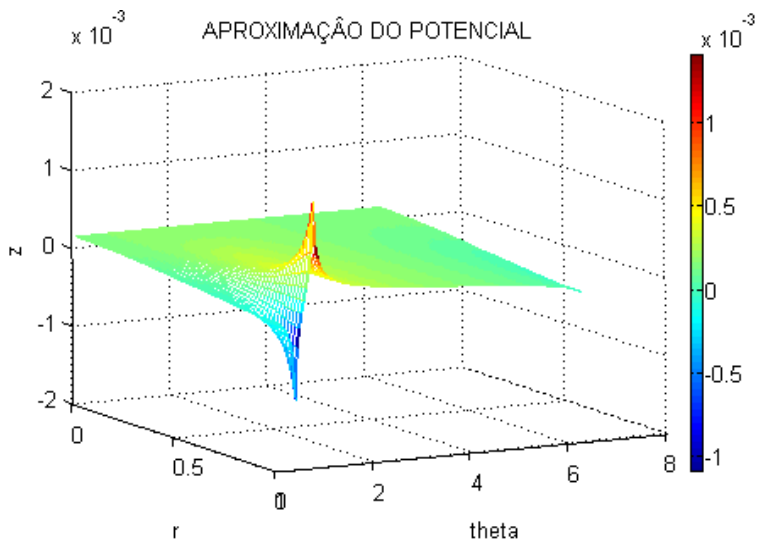

(a)

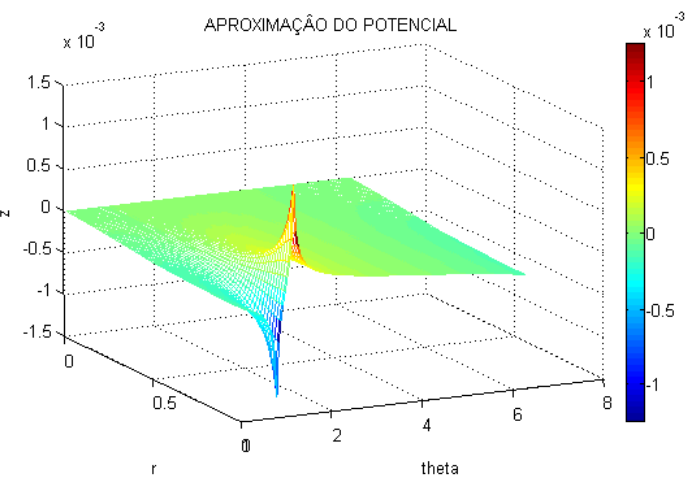

(b) 


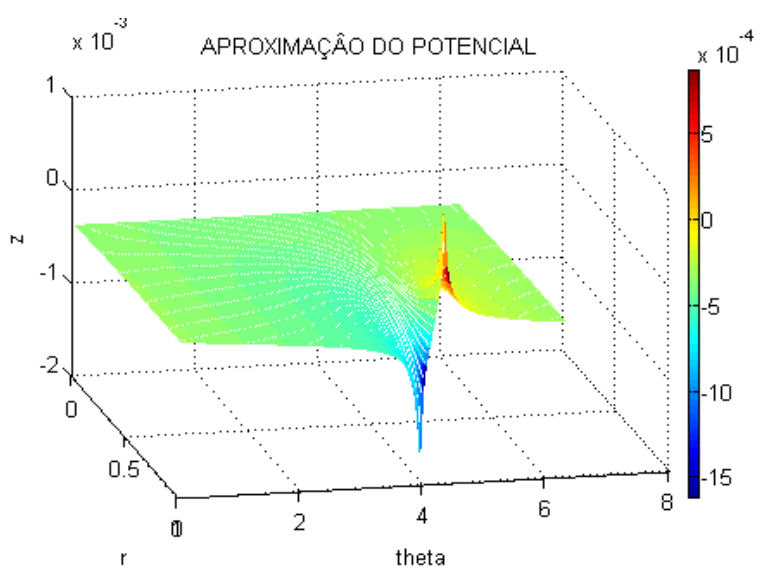

(c)

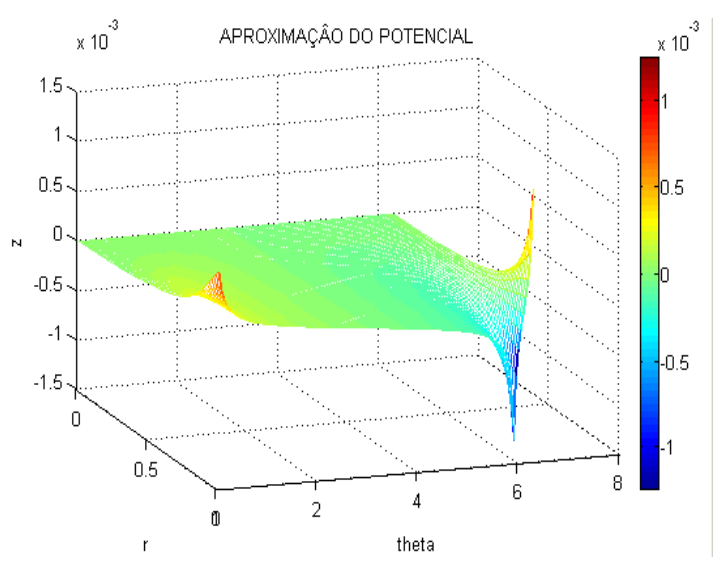

(d)

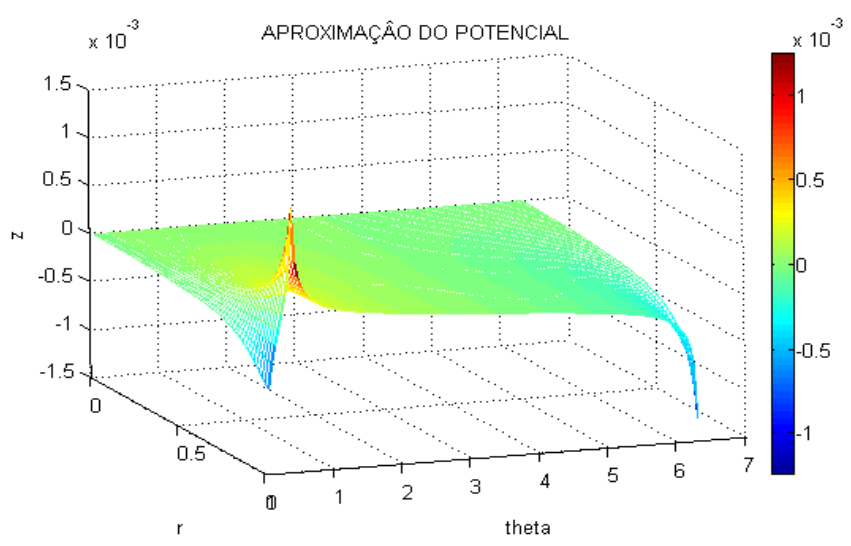

(e)

Figura 5.2 Distribuição do potencial devido á injeção de corrente adjacente : (a)1-padrão (b) 2-padrão, (c)10-padrão (d)15-padrão e (e)16 - padrão.

Na figura 5.2 podemos observar às distribuições dos potencias devido á injeção de alguns padrões de corrente do tipo adjacente.

Tabela 5.1 Erro das voltagens nos eletrodos quando usamos normalização e voltagem de referencia na solução do sistema linear (5.1) e injeção adjacente.

\begin{tabular}{|c|c|}
\hline Padrão & Erro \\
\hline$I^{1}$ & $6.1238339 \times 10^{-4}$ \\
\hline$I^{2}$ & $6.3277224 \times 10^{-4}$ \\
\hline$I^{10}$ & 0.0015079 \\
\hline$I^{15}$ & $8.7902106 \times 10^{-4}$ \\
\hline$I^{16}$ & $6.3581059 \times 10^{-4}$ \\
\hline
\end{tabular}


Na tabela 5.1 podemos observar o erro quando comparamos ás voltagens nos eletrodos usando normalização pela fronteira e o método que considera um nó de referência para resolver o sistema linear.

\section{PULA 1}

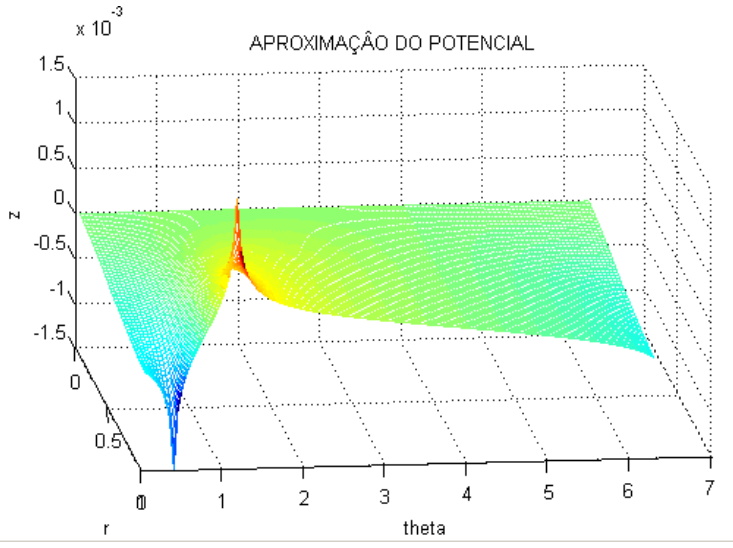

(a)

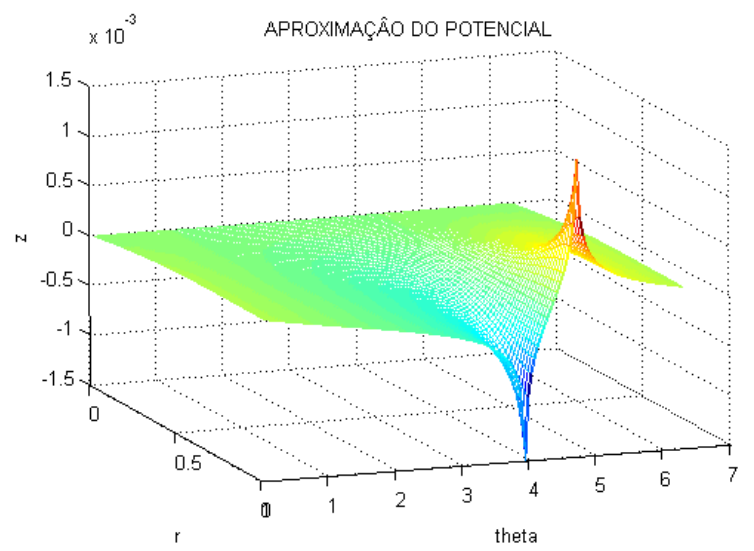

(c)

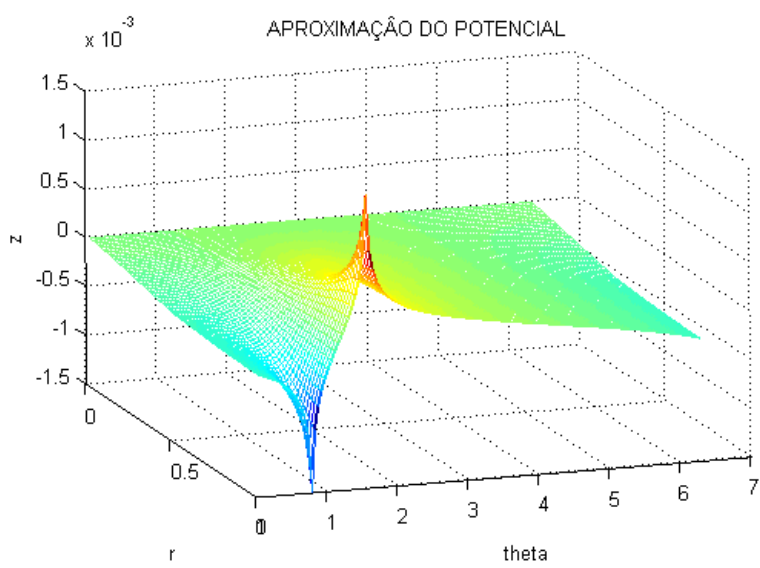

(b)

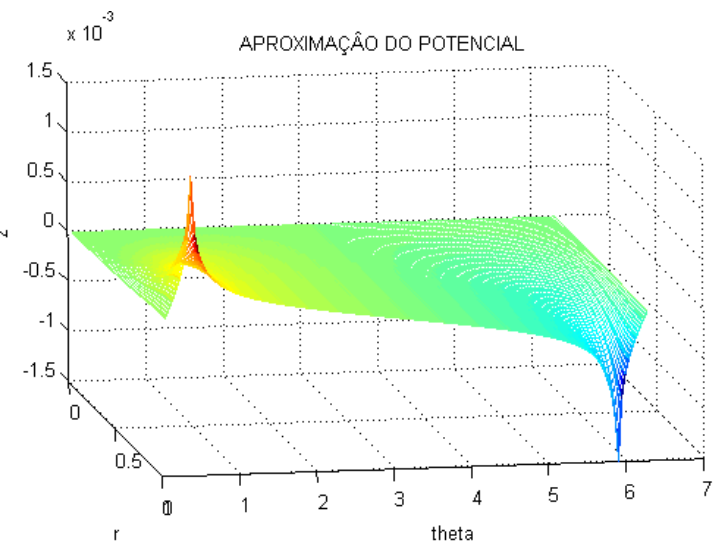

(d)

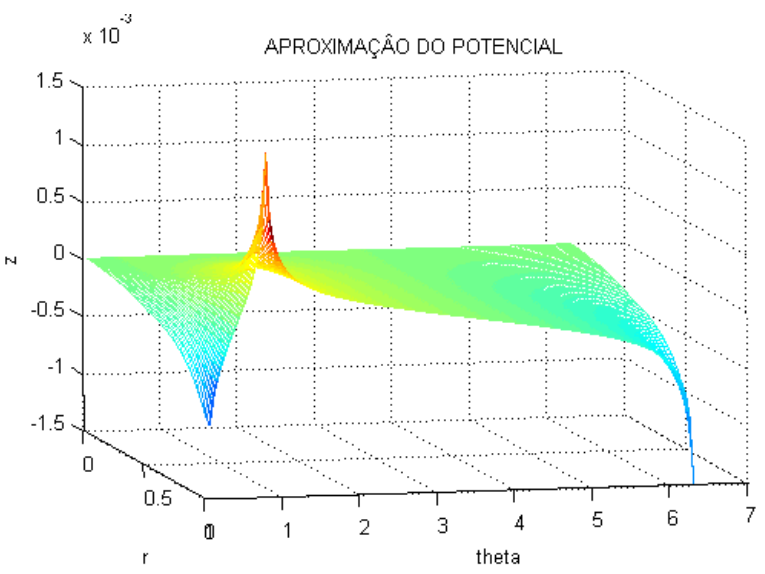

(e)

Figura 5.3 Distribuição do potencial devido á injeção de corrente pula 1: (a)1-padrão (b) 2-padrão, (c)10-padrão (d)15-padrão e (e)16 - padrão. 
Na figura 5.3 observamos algumas distribuições dos potencias devido á injeção de padrões de corrente do tipo pula 1.

Tabela 5.2 Erro das voltagens nos eletrodos quando usamos normalização e voltagem de referencia na solução do sistema linear (5.1) e injeção pula1.

\begin{tabular}{|c|c|}
\hline Padrão & Erro \\
\hline$I^{1}$ & $6.3145672 \times 10^{-4}$ \\
\hline$I^{2}$ & $6.5431231 \times 10^{-4}$ \\
\hline$I^{10}$ & $7.0987653 \times 10^{-4}$ \\
\hline$I^{15}$ & $9.8106123 \times 10^{-4}$ \\
\hline$I^{16}$ & $6.4187492 \times 10^{-4}$ \\
\hline
\end{tabular}

Na tabela 5.2, observamos o erro cometido quando comparamos ás voltagens nos eletrodos usando normalização pela fronteira e também considerando um nó de referência para resolver o sistema linear. Destas informações podemos dizer que ambos os métodos produzem aproximações satisfatórias e podem ser usados para termos uma única solução do sistema linear 5.1.

\subsection{O Jacobiano}

Como descrito no capitulo 2 , seção 2.4, para resolver o problema inverso usando alguma técnica de linearização precisamos calcular eficientemente o Jacobiano ou matriz de sensibilidade, dado que este calculo gasta uma parcela grande do tempo computacional total.

Em [67] podemos encontrar uma metodologia para calcular o Jacobiano chamado de método standard, onde:

$$
J\left(\sigma_{0}\right)=\frac{\partial U\left(\sigma_{0}\right)}{\partial \sigma}=\left.\frac{\partial\left[(A G(\sigma))^{-1} I^{d}\right]}{\partial \sigma}\right|_{\sigma=\sigma_{0}},
$$

é o Jacobiano dada uma distribuição de condutividade $\sigma_{0}$.

Mas, sabemos que: 


$$
A G(\sigma)(A G(\sigma))^{-1}=I
$$

e derivado com respeito das variáveis $\sigma$ :

$$
\frac{\partial(A G)}{\partial \sigma}(A G)^{-1}+(A G) \frac{\partial(A G)^{-1}}{\partial \sigma}=0
$$

então:

$$
(A G) \frac{\partial(A G)^{-1}}{\partial \sigma}=-\frac{\partial(A G)}{\partial \sigma}(A G)^{-1}
$$

em conseqüência:

$$
\frac{\partial(A G)^{-1}}{\partial \sigma}=-(A G)^{-1} \frac{\partial(A G)}{\partial \sigma}(A G)^{-1}
$$

Assim, uma maneira de calcular o Jacobiano é dada como:

$$
J_{d, i}=-\left.(A G)^{-1} \frac{\partial(A G)}{\partial \sigma_{i}}(A G)^{-1} I^{d}\right|_{\sigma=\sigma_{0}}
$$

onde:

$J_{d, i}=\mathrm{O}$ Jacobiano em função das condutividades $\sigma_{i}, i=1,2,3, \ldots$, ne, onde ne é o número total de volumes finitos, e $d=1,2,3, . ., P$ são os padrões de corrente.

$A G^{-1}=$ Inversa da matriz do sistema que resolve o problema direto (5.1). A matriz $A G$, a qual é dada em função das variáveis de condutividade $\sigma_{i}$, é chamada de matriz simbólica.

$\frac{\partial(A G)}{\partial \sigma_{i}}=$ Derivada parcial da matriz de condutividade simbólica respeito das variáveis $\sigma_{i}$. $I^{d}=$ Padrões de corrente, $d=1,2,3, . ., P$.

Também podemos observar que no cálculo da matriz $\frac{\partial(A G)}{\partial \sigma_{i}}$ somente alguns poucos elementos da matriz simbólica $A G$ são diferentes de zero, no máximo 13 elementos. Assim o 
cálculo de $\frac{\partial(A G)}{\partial \sigma_{i}}(A G)^{-1} I^{d}$ tem que ser feito convenientemente para otimizar o tempo computacional.

Para extrair os valores das voltagens correspondentes aos 16 eletrodos temos que introduzir uma matriz $T \in \mathfrak{R}^{L x(M+1) N}$.

$$
T=\left[\begin{array}{cccccc}
1 & 0 & \cdots & 0_{(1, l)} & \cdots & 0_{(1, s)} \\
0 & 1 & \cdots & 0_{(2, l)} & \cdots & 0_{(2, s)} \\
\vdots & \vdots & \ddots & \vdots & \ddots & \vdots \\
0 & 0 & \cdots & 1_{(l, l)} & \cdots & 0_{(l, s)}
\end{array}\right]_{L x(M+1) N},
$$

assim o vetor de aproximações das voltagens na fronteira é:

$$
v_{d}(\sigma)=h_{d}(\sigma)=(A G)^{-1} I^{d}
$$

onde $h_{d}$ é um operador não linear.

Aproximando a transformação $h_{d}$ pela serie de Taylor ate os termos de primeira ordem:

$$
v_{d}(\sigma) \simeq v_{d}\left(\sigma_{0}\right)+\left.\frac{\partial h_{d}}{\partial \sigma}\right|_{\sigma=\sigma_{0}}\left[\sigma-\sigma_{0}\right]
$$

e

$$
\left.J_{d}\left(\sigma_{0}\right) \simeq \frac{\partial h_{d}}{\partial \sigma}\right|_{\sigma=\sigma_{0}}=-(A G)^{-1} \frac{\partial(A G)}{\partial \sigma}(A G)^{-1} I^{d}
$$

então:

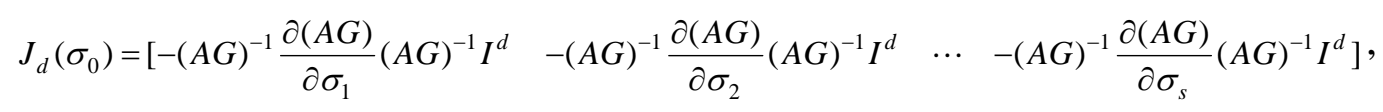

onde $d=1,2,3, . ., P$.

Quando consideramos a matriz $T$ resulta: 


$$
T v_{d}(\sigma)=T v_{d}\left(\sigma_{0}\right)+\left[-T(A G)^{-1} \frac{\partial(A G)}{\partial \sigma_{1}}(A G)^{-1} I^{d} \quad-T(A G)^{-1} \frac{\partial(A G)}{\partial \sigma_{2}}(A G)^{-1} I^{d} \quad \cdots \quad-T(A G)^{-1} \frac{\partial(A G)}{\partial \sigma_{s}}(A G)^{-1} I^{d}\right]\left[\sigma-\sigma_{0}\right]
$$

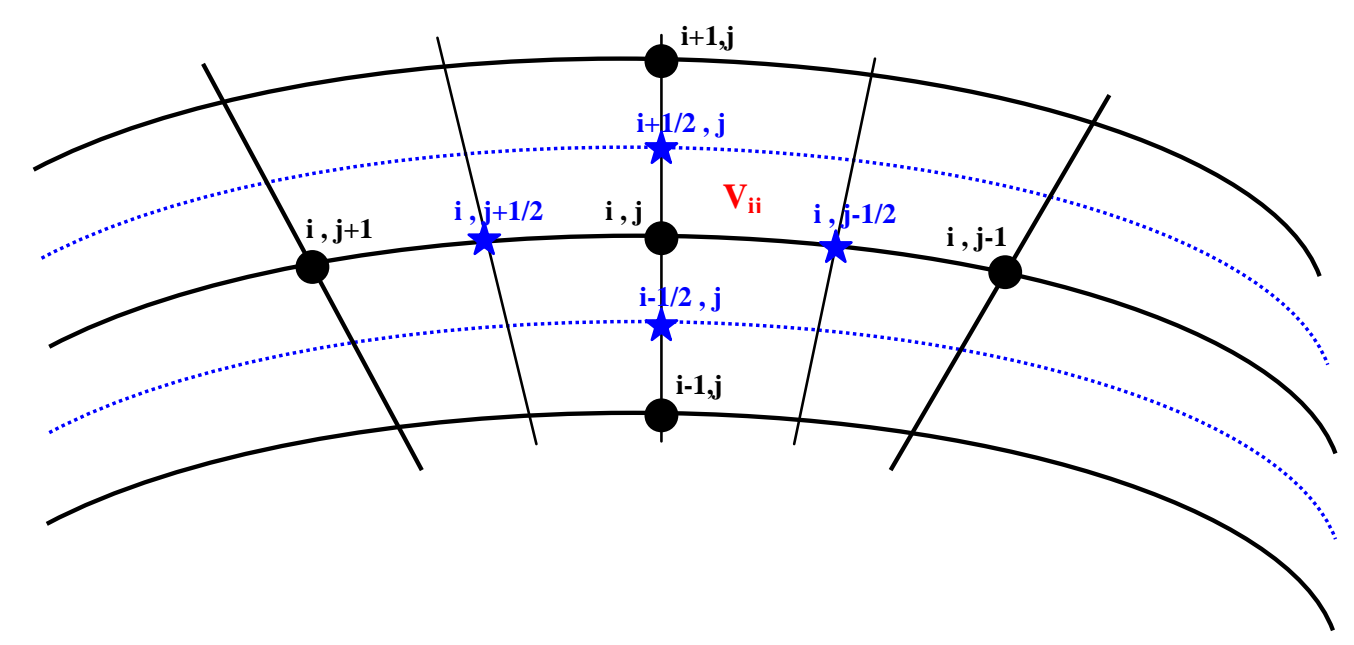

Figura 5.4 Volume finito $V_{i j}$ correspondente á variável de condutividade $\sigma_{i j}$.

Calculado o Jacobiano, temos que resolver o sistema:

$$
J\left(\sigma_{i}\right)(\delta u)=\delta V
$$

onde:

$$
\delta u=u-u_{i}, \delta V=V(\sigma)-V\left(\sigma_{i}\right)
$$

Porém, o sistema (5.3) é mal condicionado, pois o numero de condição do Jacobiano é da ordem $10^{20}$. Para lidar com o mal condicionamento é preciso usar técnicas de regularização como por exemplo o método de Tikhonov [59].

\subsection{Aproximação do Fluxo nos nós}

As expressões das componentes do fluxo nas laterais dos volumes finitos (ver figura 5.4) são dadas como: 


\section{Método HIA}

$\left(W_{1}\right)_{i+1 / 2 j}=\frac{1}{r_{i+1 / 2}}\left\{\frac{-\left[r_{i+1 / 2} K_{i+1 / 2 j}^{H} \frac{u_{i+1 j}-u_{i j}}{h_{r}}\right]\left(1-a_{i-1 / 2 j}\right)-\left[r_{i-1 / 2} K_{i-1 / 2 j}^{H} \frac{u_{i j}-u_{i-1 j}}{h_{r}}\right] a_{i+1 / 2 j}}{\left(1+a_{i+1 / 2 j}\right)\left(1-a_{i-1 / 2 j}\right)+a_{i+1 / 2 j} a_{i-1 / 2 j}}\right\}$

e

$$
\left(W_{2}\right)_{i j+1 / 2}=\frac{-\left[K_{i j+1 / 2}^{H} \frac{u_{i j+1}-u_{i j}}{r_{i} h_{\theta}}\right]\left(1-a_{i j-1 / 2}\right)-\left[K_{i j-1 / 2}^{H} \frac{u_{i j}-u_{i j-1}}{r_{i} h_{\theta}}\right] a_{i j+1 / 2}}{\left(1+a_{i j+1 / 2}\right)\left(1-a_{i j-1 / 2}\right)+a_{i j+1 / 2} a_{i j-1 / 2}} .
$$

\section{Método HA}

$$
\left(W_{1}\right)_{i+1 / 2 j}=-K_{i+1 / 2 j}^{H} \frac{u_{i+1 j}-u_{i j}}{h_{r}}
$$

e

$$
\left(W_{2}\right)_{i j+1 / 2}=-K_{i j+1 / 2}^{H} \frac{u_{i j+1}-u_{i j}}{r_{i} h_{\theta}} .
$$

Para aproximar os termos dos fluxos nos nós fazemos:

$$
\begin{aligned}
& \left(W_{1}\right)_{1 / 2 j}=\frac{1}{N} \sum_{j=1}^{N}\left(W_{1}\right)_{3 / 2 j} \\
& \left(W_{1}\right)_{i j}=\left\{\begin{array}{l}
\frac{\left(W_{1}\right)_{3 / 2 j}+\left(W_{1}\right)_{1 / 2 j}}{2}, i=1 \\
\frac{\left(W_{1}\right)_{i+1 / 2 j}+\left(W_{1}\right)_{i-1 / 2 j}}{2}, i=2,3, \ldots, M
\end{array}\right. \\
& \left(W_{2}\right)_{i 1 / 2}=-\left(K_{i 1 / 2}^{H} \frac{u_{i 1}-u_{i N}}{r_{i} h_{\theta}}\right) \\
& \left(W_{2}\right)_{i j}=\left\{\begin{array}{l}
\frac{\left(W_{2}\right)_{i 3 / 2}+\left(W_{1}\right)_{i 1 / 2}}{2}, j=1 \\
\frac{\left(W_{2}\right)_{i j+1 / 2}+\left(W_{2}\right)_{i j-1 / 2}}{2}, j=2,3, \ldots, N-1 \\
\frac{\left(W_{2}\right)_{i 1 / 2}+\left(W_{1}\right)_{i N-1 / 2}}{2}, j=N
\end{array}\right.
\end{aligned}
$$


Tabela 5.3 Erro do fluxo na direção radial do problema 5.2 usando o método HA.

\begin{tabular}{|c|c|c|}
\hline $\begin{array}{c}\text { GRID } \\
\mathbf{M}\end{array}$ & $\begin{array}{c}\text { Erro máximo do fluxo } \\
\text { na direção radial }\end{array}$ & $t x_{c}$ \\
\hline $\mathbf{1 6}$ & 0.0057678 & - \\
\hline $\mathbf{3 2}$ & 0.0034065 & 0.76 \\
\hline $\mathbf{6 4}$ & 0.0018296 & 0.89 \\
\hline $\mathbf{1 2 8}$ & $9.4586611 \times 10^{-4}$ & 0.95 \\
\hline
\end{tabular}

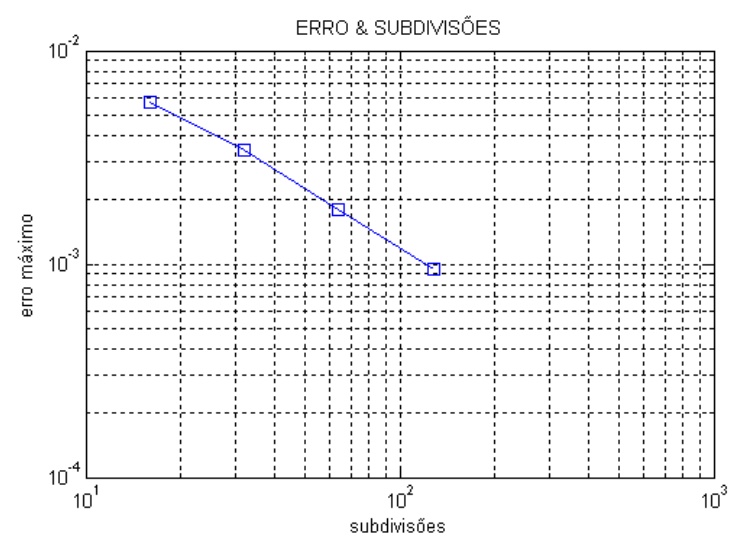

Figura 5.5 Erro máximo do fluxo na direção radial .

Na tabela 5.3 e na figura 5.5 podemos ver que o método HA em coordenadas polares aproxima o fluxo com ordem 1. Espera-se que aproximações mais acuradas, por exemplo, aquelas provenientes de usar o método HIA aproximem o fluxo com ordem 2, o qual é verdade em coordenadas cartesianas [14].

\subsection{O Jacobiano em temos do Fluxo}

Com o objetivo de poupar tempo computacional no cálculo do Jacobiano, apresentamos uma metodologia baseada nas componentes do fluxo.

Consideramos a solução da equação de difusão (2.13) junto à condição de fronteira (2.19) e consideramos também uma condição de fronteira chamada de dipolo definida como: 


$$
\Upsilon^{l}=\left\{\begin{array}{l}
\frac{-1}{\left|e_{l}\right|}, x \in e_{l}, l=1,2, . ., L \\
\frac{1}{\left|e_{l}\right|}, x \in e_{l+1}, l=1,2, . ., L . \\
0, x \in \partial \Omega \backslash \bigcup_{l=1}^{L} e_{l}
\end{array}\right.
$$

Com estas considerações e usando o teorema de divergência e algumas outras hipóteses ${ }^{1}$, temos que as componentes do Jacobiano ficam da seguinte maneira:

$$
J_{d l, k}=\frac{\partial V_{d l}}{\partial \sigma_{k}}=-\iint_{\Omega_{k}} \nabla u\left(\Upsilon^{l}\right) \cdot \nabla u\left(I^{d}\right) d A,
$$

onde $u\left(\Upsilon^{l}\right), l=1,2,3, \ldots, L$ corresponde à solução do problema direto devido á condição de fronteira dipolo e $u\left(I^{d}\right)$ corresponde á distribuição do potencial quando usamos o padrão de corrente $I^{d}, d=1,2,3, \ldots, P$.

Mas, sabemos que:

$$
\left\{\begin{array}{l}
-\sigma \nabla u\left(I^{d}\right)=W^{I} \\
-\sigma \nabla u\left(\Upsilon^{l}\right)=W^{\Upsilon}
\end{array}\right.
$$

onde lembramos que as componentes de um fluxo $W=-\sigma \nabla u$ são:

$$
W(r, \theta)=\left[\begin{array}{c}
-\sigma u_{r} \\
-\frac{\sigma}{r} u_{\theta}
\end{array}\right],
$$

\footnotetext{
${ }^{1}$ Os detalhes de (5.4) são dados no apêndice B.
} 
então, em termos dos fluxos $W^{I}, W^{\Upsilon}$ temos:

$$
W^{I}=\left[\begin{array}{l}
W_{1}^{I} \\
W_{2}^{I}
\end{array}\right], W^{\Upsilon}=\left[\begin{array}{l}
W_{1}^{\Upsilon} \\
W_{2}^{\Upsilon}
\end{array}\right],
$$

assim:

$$
\nabla u\left(\Upsilon^{l}\right) \cdot \nabla u\left(I^{d}\right)=\frac{\partial u\left(\Upsilon^{l}\right)}{\partial r} \frac{\partial u\left(I^{d}\right)}{\partial r}+\frac{1}{r^{2}} \frac{\partial u\left(\Upsilon^{l}\right)}{\partial \theta} \frac{\partial u\left(I^{d}\right)}{\partial \theta}=\frac{W_{1}^{\Upsilon} W_{1}^{I}}{\sigma^{2}}+\frac{W_{2}^{\Upsilon} W_{2}^{I}}{\sigma^{2}} .
$$

Portanto:

$$
\begin{aligned}
\int_{r_{i-1 / 2}}^{r_{i+1 / 2}} \int_{\theta_{j-1 / 2}}^{\theta_{j+1 / 2}} \nabla u\left(\Upsilon^{l}\right) \cdot & \nabla u\left(I^{d}\right) v d A=\int_{r_{i-1 / 2}}^{r_{i+1 / 2}} \int_{\theta_{j-1 / 2}}^{\theta_{j+1 / 2}} \frac{W_{1}^{\Upsilon} W_{1}^{I}}{\sigma^{2}}+\int_{r_{i-1 / 2}}^{r_{i+1 / 2}} \int_{\theta_{j-1 / 2}}^{\theta_{j+1 / 2}} \frac{W_{2}^{\Upsilon} W_{2}^{I}}{\sigma^{2}} \\
& \simeq \frac{1}{\sigma_{i j}^{2}} h_{r} h_{\theta}\left(r W_{1}^{\Upsilon} W_{1}^{I}\right)_{i j}+\frac{1}{\sigma_{i j}^{2}} h_{r} h_{\theta}\left(r W_{2}^{\Upsilon} W_{2}^{I}\right)_{i j} \\
& =\frac{1}{\sigma_{i j}^{2}} h_{r} h_{\theta} r_{i}\left(W_{1_{i j}}^{\Upsilon} W_{2_{i j}}^{I}+W_{1_{i j}}^{\Upsilon} W_{2_{i j}}^{I}\right)
\end{aligned} .
$$

Finalmente, a expressão do Jacobiano em termos do fluxo fica:

$$
J_{d l, k}=-\frac{1}{\sigma_{i j}^{2}} h_{r} h_{\theta} r_{i}\left(W_{1_{i j}}^{\Upsilon} W_{1_{i j}}^{I}+W_{2_{i j}}^{\Upsilon} W_{2_{i j}}^{I}\right)
$$

onde os sub - índices ij relacionam um volume finito $V_{k}$, como mostra a figura 5.4.

Na tabela 5.4 comparamos o desempenho no cálculo do Jacobiano em termos do fluxo e quando usamos o método standard em uma distribuição homogênea de condutividade. 
Tabela 5.4 Tempo computacional no cálculo do Jacobiano.

\begin{tabular}{|c|c|c|}
\hline $\begin{array}{c}\text { Volumes } \\
\text { Finitos }\end{array}$ & Em termos do fluxo & Método standard \\
\hline $\mathbf{M}^{* \mathbf{N}}$ & Tempo(s) & Tempo(s) \\
\hline 512 & 1.015625 & 2.96125 \\
\hline 2048 & 3.921875 & 38.6175 \\
\hline 8192 & 18.203125 & 185.5615 \\
\hline
\end{tabular}

Na tabela 5.4 podemos observar que o cálculo do Jacobiano com a metodologia que usa os termos das componentes do fluxo gasta aproximadamente 10 vezes menos tempo computacional daquele que usa o método standard.

\subsection{Aproximação Gauss - Newton}

Para resolver o problema inverso usamos a aproximação Gauss - Newton com regularização de Tikhonov [59], onde consideramos as seguintes informações:

Aproximação inicial $\sigma_{0}$ : Por se tratar de um problema com dados numéricos o valor máximo e mínimo da condutividade é conhecido. Assim consideramos $\sigma_{0}=(\max (\sigma)+\min (\sigma)) / 2$. Em problemas reais também é possível conhecer os valores máximos e mínios das variáveis. Uma outra alternativa para se obter uma primeira distribuição de condutividade é dada em (3.12).

Injeção de corrente: Neste problema consideramos padrões de corrente do tipo pula 1.

Parâmetro de regularização $\alpha$ e matriz de regularização $R$ : Estas informações são dadas a priori e geralmente se usam as informações das variáveis do problema especifico.

Para resolver o problema direto usamos o esquema HA com normalização pela fronteira e para calcular o Jacobiano usamos a metodologia que usa as componentes do fluxo.

O processo iterativo de Gauss - Newton com regularização de Tikhonov tem a seguinte forma: 


$$
\begin{aligned}
& \text { para } \mathrm{i}=1 \text { : max it } \\
& \sigma_{\mathrm{i}}=\sigma_{i-1}+\delta \sigma_{i-1} \\
& \delta \sigma_{i-1}=\left(J_{i-1}^{T} J_{i-1}+\alpha R^{T} R\right)^{-1}\left[J_{i-1}^{T}\left(V-U_{\sigma_{i-1}}\right)-\alpha R^{T} R \sigma_{i-1}\right]
\end{aligned}
$$

onde max it é um número máximo, pré-especificado de iterações.

\section{Resultados:}

Usando: $\alpha=10^{-3}, \quad \sigma_{0}=(\max (\sigma)+\min (\sigma)) / 2$ e 10 iterações. O problema é reconstruir a seguinte distribuição de condutividade:

$$
\sigma(r, \theta)=\left\{\begin{array}{l}
0.1, \quad 0<r<r_{m}, \quad 0 \leq \theta \leq 2 \pi \\
0.3, \quad r_{m} \leq r \leq 1, \quad 0 \leq \theta \leq 2 \pi \\
r_{m}=r_{M / 2}+0.5 r_{M+1}
\end{array}\right.
$$

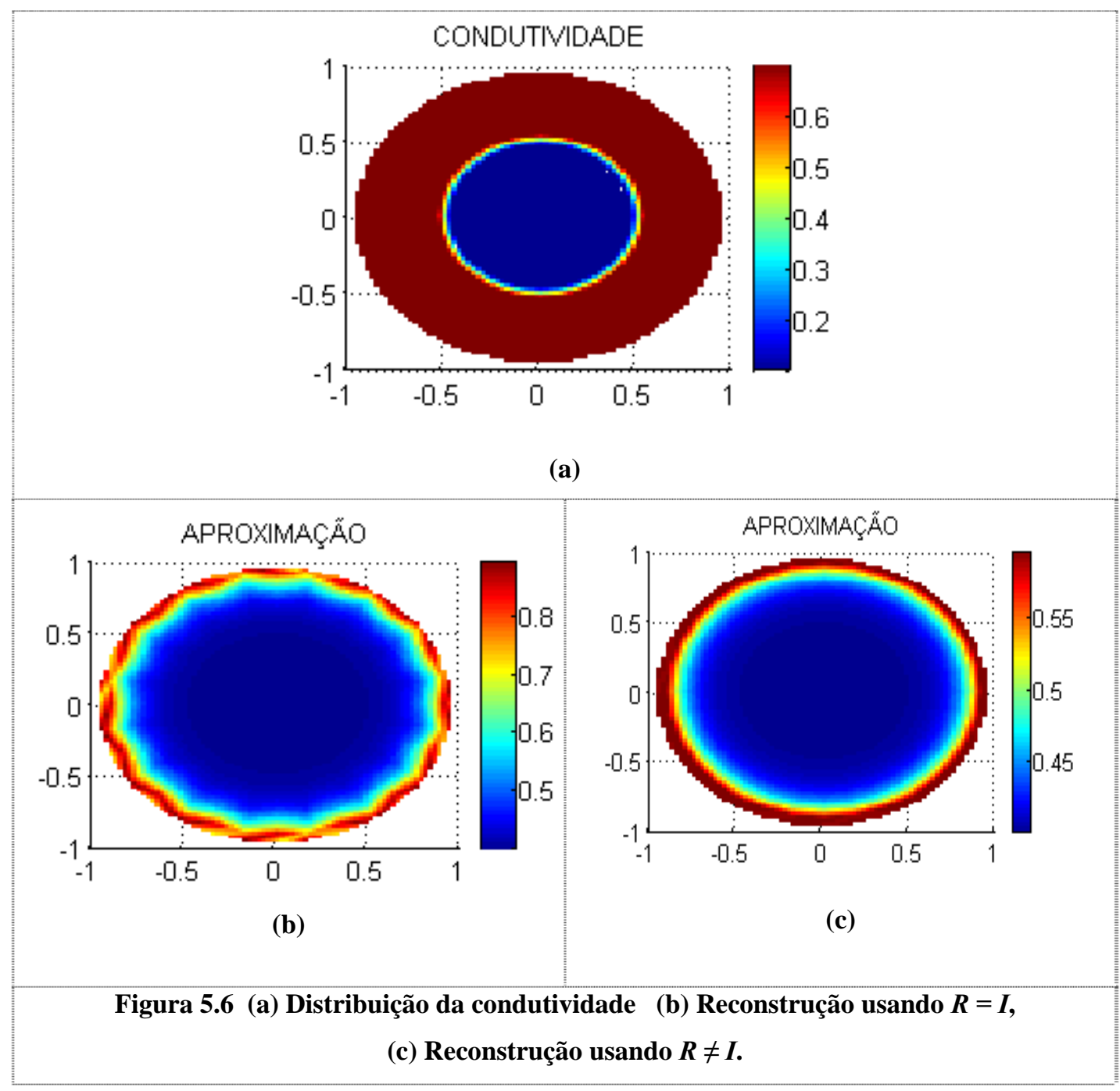


A figura 5.5 mostra a reconstrução de condutividade $\sigma$, onde consideramos duas matrizes de regularização. A figura 5.5 (a) usa a matriz de regularização $R=I$ e a figura 5.5 (b) considera uma matriz de regularização $R$ com pesos, a qual uniformiza as mudanças bruscas de condutividade em regiões circulares. O tempo computacional total que usa a metodologia descrita para se obter uma reconstrução de condutividade é aproximadamente 17s e o erro das voltagens nos eletrodos quando usamos a distribuição de condutividade exata e a reconstrução correspondente é de aproximadamente 0.001 .

Da figura 5.5 podemos dizer que os métodos usados para calcular o problema direto e inverso produzem resultados razoáveis. Em ambos os casos à interface é localizada. O parâmetro e as matrizes de regularização têm uma relação direta com o tipo de resultado a ser obtido. Em algumas aplicações se precisa de imagens com uma resolução onde não se considere as mudanças bruscas de condutividade (resolução priorizando suavidade), já em outras aplicações as mudanças de condutividade (ou resistividade) trazem informações valiosas [11]. 


\section{6.- CONCLUSÕES}

Nos capítulos 1 e 2 formulamos a técnica da TIE para a reconstrução de imagens da distribuição da condutividade ou resistividade do interior de um domínio condutor. Assim precisamos resolver o problema direto e inverso numericamente. Também temos visto que o sucesso da técnica da TIE depende fortemente de resolver o problema direto eficientemente, pois ele é “chamado" varias vezes quando o problema inverso é resolvido como um processo iterativo. Por esta razão, no capitulo 4, se desenvolveu algumas técnicas numéricas para aproximar o problema direto onde as variáveis (condutividade ou resistividade) tem diferentes propriedades como de serem contínuas ou descontínuas. Assim, propomos alguns métodos de solução usando métodos numéricos como diferenças finitas, volumes finitos e volumes finitos modificados, onde podemos concluir que o uso destes métodos numéricos produz aproximações de segunda ordem. Os métodos propostos para tratar o problema de descontinuidade das variáveis em coordenadas cartesianas e polares (HA e HIA), são duas formas de abordar a aproximação numérica do problema direto tendo cada uma suas vantagens e desvantagens como custo computacional, tratamento da matriz do sistema linear e erro de convergência. Outra propriedade interessante na metodologia adotada para a aproximação das variáveis é o calculo nodal (e não por elementos), o qual se traduz em poupar tempo computacional.

Também temos visto que o calculo do Jacobiano (ou matriz de sensibilidade) é essencial na TIE, pois esse cálculo gasta uma parcela grande do tempo computacional total. Assim propomos uma maneira de calcular o Jacobiano em termos do fluxo o qual otimiza o tempo computacional total.

Durante o desenvolvimento do trabalho nos deparamos com a necessidade de generalizar a aproximação numérica do problema direto em domínios onde sejam possíveis aplicações mais realistas. Assim surgiram algumas situações para estudos posteriores, por exemplo, a modelagem na fronteira levando em consideração o contato de impedância e o fato da voltagem ser constante nos eletrodos o qual resulta na formulação do modelo completo de eletrodos. Também se apresentou a tarefa de aplicar a metodologia que usa a TIE em 
domínios com fronteira que podem ser uma deformação do disco o qual conduz a aplicações médicas mais realistas. Contudo, testes onde se use dados experimentais provenientes de um sistema de tomografia por impedância elétrica devem validar os nossos códigos desenvolvidos. 


\section{REFERÊNCIAS BIBLIOGRÁFICAS}

[1] Adler. A, Lionheart.R, Uses and abuses of EIDORS:An extensible software base for EIT, Manchester Institute for Mathematical Sciences, The University of Manchester, UK, 2008.

[2] Borcea. L, Electrical impedance tomography, Inverse Problems, pp. 99 -136, 2002.

[3] Barber. D, Brown. B, Errors in reconstruction of resistivity images using a linear reconstruction technique, Clinical Physics and Physiological Measurement 9, pp. 101104, 1988.

[4] Barber. D, Brown. B, Applied potential tomography, Journal of Physical Engineering: Science Instrumentation 17, pp. 723-733 (1984).

[5] Barber. D, Brown. B, Freeston. I, Imaging spatial distributions of resistivity using potential tomography, Electronic Letters 19, pp. 993-935, 1983.

[6] Betrero. M, Boccacci. P, Introduction to inverse problems in imaging, IoP, Bristol, 1998.

[7] Breckon. W, Image reconstruction in Electrical Impedance Tomography, Ph.D. thesis, Oxford polytechnic, Oxford, 1990.

[8] Calderon. A, On an inverse boundary value problem, In the proceeding of a conference on Numerical Analysis and Its Applications to Continuum Physics, pp. 67-73, 1980.

[9] Cheney. M, Isaacson. D, Distinguishability in impedance imaging, IEEE Transactions in Biomedical Engineering 39, pp. 852-860, 1992.

[10] Cheney. M, Isaacson. D, Newell. J. et al, NOSER: An algorithm for solving the inverse conductivity problem, International Journal of Imaging Systems and Technology 2, pp. 66-75, 1990.

[11] De Lima. C, Estudo da obtenção de imagens de tomografia por impedância elétrica do pulmão pelo método de otimização topológica, Ph.D, tese de doutoramento, POLIUSP, 2006.

[12] Dorn. O, Miller. E, Rappaport. C, A shape reconstruction methods for electromagnetic tomography using adjoint fields and level set, Inverse Problems 16, no 5, pp. 11191156, 2000.

[13] Erol. R, Mangnall. Y, et al, Identifying esophageal contents using electrical impedance tomography, Physiological Measurement, pp. 253-261, 1995.

[14] Ewing. R, Iliev. O, Lazarov. R, A modified finite volume approximation of secondorder elliptic equations with discontinuous coefficients, SIAM J. Scientific Computational, pp.1335-1351, 2001. 
[15] George. P, Mahdi. M, et al., Electrical impedance tomography (EIT) in pulmonary measurement: a review of applications and research, Proceedings of the fifth IASTED International Conference: biomedical engineering, pp. 221-230, 2007.

[16] Hadamard. J, Le problème de Cauchy, Hemann. Edit., Paris, 1932.

[17] Hansen. P, Regularization tools - A Matlab package for analysis and solution of Discrete ill-posed problems, Departament of mathematical modeling, Technical University of Denmark, Denmark, 2001.

[18] Hua. P, Woo. J, Topkins. W, Using compound electrodes in electrical impedance tomography, IEEE Transactions of Biomedical Engineering 40, pp. 335-343, 1993.

[19] Hunt. A, Pendleton. J, Ladam. Y, Visualization of two phase gas-liquid pipe flows using electrical capacitance tomography, Proceedings ESDA2004, Manchester, 2004.

[20] Iliev. O, On second-order-accurate discretization and its fast solution with a pointwise multigrid solver, IMA Journal of Numerical Analysis. pp. 391-406, 2002.

[21] Isakov. V, Uniqueness and stability in multi-dimensional inverse problems, Inverse Problems 9, pp. 579-621, 1993.

[22] Isaacson. D, Distinguishability of conductivities by electric current computed tomography, IEEE Transactions on Medical Imaging MI-5, pp. 91-95, 1986.

[23] Isaacson. D, Gisser. G, Neweel. J, Current topics in impedance imaging, Clinical Physics and Physiological Measurement 8, pp. 39-46, 1987.

[24] Jon. F, Horowitz. L, et al, Temperature dependence of tissue impedivity in electrical impedance tomography of cryosurgery, IEEE transactions on biomedical engineering, pp. 695-701, 2005.

[25] Kaasschieter. E, Preconditioned conjugated gradients for solving singular systems, Journal of computational and applied mathematics 24, pp. 265-275, 1988.

[26] Kaipio. J, Karjalainen. E, Vauhkonen,. M, State estimation in time-varying electrical impedance tomography, Annals of New York Academy of Science 873, pp. 430-439, 1999.

[27] Kleinermann. F, Avis. N, Alhargan,. F, Analytical solution to the three-dimensional electrical forward problem for an elliptical cylinder, Physiological Measurement 23, pp. 141-147, 2002.

[28] Khon. R, Vogelius. M, Determining conductivity by boundary measurements, Communications in Pure and Applied Mathematics 37, pp. 113-123, 1984.

[29] Le Veque. R, Li. Z, The immersed interface method for elliptic equations with discontinuous coefficients and singular sources, SIAM J. Numerical Analysis, pp. 1019- 1044, 1994. 
[30] Levenberg. K, A method for the solution of certain problems in least squares, SIAM Journal of Numerical Analysis 16, pp. 588-604, 1944.

[31] Lehr. J, A vector derivation useful in impedance plethysmographic field calculation, Short communications, IEEE Transactions in Biomedical Engineering, 1972.

[32] Lionheart. W, EIT reconstruction algorithms: pitfalls, challenges and recent developments, Physiological Measurement, pp. 125-142, 2004.

[33] Lionheart. W, Aridge. S, Schweiger. M, et al, Electrical Impedance and Diffuse Optical Tomography Reconstruction Software, Proceeding of the $1^{\text {st }}$ world congress on industrial process tomography, pp. 150-155, 1999.

[34] Lionheart. W, Reconstruction algorithms for permittivity and conductivity imaging, Proceeding of the $2^{\text {nd }}$ congress on industrial process tomography, pp. 4-11, 2001.

[35] Lionheart. W, Conformal uniqueness results in anisotropic electrical impedance imaging, Inverse problems 13, pp. 125-134, 1997.

[36] Ming-Chih. L, Yu-Hou. T, A fast iterative solver for the variable coefficient diffusion equation on a disk, Manuscript of Comp. Phys. pp. 1-13, 2005.

[37] Ming-Chih. L, Chin-Tien. L, Yu-Hou. T, An efficient semi-coarsening multigrid method for variable diffusion problems in cylindrical coordinates, App. Num. Math, Vol.57, Issue 5-7. pp. 801-810, 2007.

[38] Metherall. P, Barber. D, Smallwood. R, et al, Three dimensional electrical impedance tomography, Nature 380, pp. 509-512, 1996.

[39] Murai. T, Kagawa. Y, Electrical impedance computed tomography based on a finite element model, IEEE Transactions on Biomedical Engineering 32, pp. 177-184, 1985.

[40] McArdle. F, Brown. B, et al, An in - vivo examination of cardiac impedance changes imaged by cardiardiosytnchronous averaging, Clinical and physiological applications of electrical impedance tomography, London, D. Holder, 1993.

[41] Parker. L, The inverse problem of resistivity sounding, Geophysics, pp. 2143-2158, 1984.

[42] Paulson. K, Breckon. W, Pidcock. M, Electrode modeling in electrical impedance tomography, SIAM Journal of Applied Mathematics 52, pp. 1012-1022, 1992.

[43] Pidcock. M, Paulson. K, Breckon. W. A hybrid phantom for eit, CAIT workshop proceeding, York, UK, 1991.

[44] Pinheiro. P, Three-dimensional image reconstruction algorithm for electrical resistance tomography, Ph.D.thesis, UMIST, Manchester, 1994. 
[45] Polydorides. N, Lionheart. W, A MAtlab toolkit for three-dimensional electrical impedance tomography: a contribution to the EIDORS project, Measurement Science and technology 13, pp. 1871-1883, 2002.

[46] Rolnik. V, Contribuição ao desenvolvimento de uma nova técnica de reconstrução tomográfica para sondas de visualização direta, $\mathrm{Ph}$. D, tese de doutoramento, EESCUSP, 2003.

[47] Saukh. Sergey, Incomplete choleski factorization in fixed memory with flexible drop tolerance strategy, IEEE International workshop on intelligent data acquisition and advanced computing systems: Technology and applications, Ukraine, pp. 87 - 93, 2003.

[48] Samarskii, A., A., Theory of difference schemes, Moscou, Nauka, 2001.

[49] Sylvester. J, Uhlmann. G, A global uniqueness theorem for an inverse boundary value problem, Annals of Mathematics 125, pp. 153-169, 1987.

[50] Schweiger. M, Arridge. S, Delpy. D, Applications of the finite element method for the forward and inverse models in optical tomography, Journal of Mathematical Imaging and Vision 3, pp. 263-283, 1993.

[51] Seyidmamedov, Z. Ebru. O, A mathematical model and numerical solution of interface problems for steady state heat conduction, Mathematical Problems in Engineering, pp. 1-18, 2006.

[52] Sylvester. J, Uhlmann. G, A global uniqueness theorem for an inverse boundary value problem, Annals of Mathematics 125, pp. 153-169, 1987.

[53] Somersalo. E, Cheney. M, Isaacson. D, Existence and uniqueness for electrode models for electric current computed tomography, SIAM Journal of Applied Mathematics 52, pp. 1023-1040, 1992.

[54] Schweiger. M, Arride. S, Delpy. D, Applications of the finite element method for the forward and inverse models in optical tomography, Journal of Mathematical Imaging and Vision 3, pp. 263-283, 1993.

[55] Tanaka. H, Siqueira. R, et al, Fuzzy modeling of electrical impedance tomography images of the lungs, Clinics, pp. 363-370, 2007.

[56] Tikhonov. A, Arsenin. V, Solutions of ill-posed problems, Winston, Washington DC, 1997.

[57] Vaukhonen. M, Electrical impedance tomography and prior information, Ph.D. thesis, Kuopio University, Kuopio, 1997.

[58] Vaukhonen. M, Karjalainen. P, Kaipio. J, A Kalman filter approach to track fast Impedance changes in electrical impedance tomography, IEEE Transactions of Biomedical Engineering 45, pp. 486-493, 1998. 
[59] Vaukhonen. M, Vadàsz. D, Karjalainen. P, et al , Tikhonov regularization and prior information in electrical impedance tomography, IEEE Transactions on Medical Imaging 17, no 2, pp. 285-293, 1998.

[60] Vaukhonen. M, Lionheart. M, Heikkinen. L, et al. A Matlab package for the EIDORS project to reconstruct two dimensional EIT images, Physiological Measurement 22, pp. 107-111, 2001.

[61] Wang. M, Inverse solutions for electrical impedance tomography based on conjugate gradients methods, Meas. Sci. Technol. pp. 101-117, 2002.

[62] Wang. M, Electrical impedance tomography on conducting walled process vessels, Ph.D. thesis, UMIST, Manchester, 1994.

[63] Webster. J, Electrical impedance tomography, Adam Hilger, Bristol, 1990.

[64] Woo. E, Webster. J, Tompkins. W, Measuring lung resistivity using electrical impedance tomography, IEEE Transactions in Biomedical Engineering 39, pp. 756760, 1992.

[65] Wright. M, Why a pure primal Newton barrier step may be infeasible, Tech, report, AT \& Bell Laboratories, New Jersey, 1993.

[66] Yousef. S, Iterative Methods for Sparse Linear Systems, SIAM, $2^{\text {nd }}$. Ed, 2007.

[67] Yorkey. T, Webster. J, Tompkins. W, Comparing reconstruction algorithms for electrical impedance tomography, IEEE Transactions in Biomedical Engineering 34, pp. 843 - 852, 1972.

[68] Zhilin. W, Wei-Cheng. W, I-Lang. C. et al, New formulation for interface problems in polar coordinates, SIAM Journal on Scientific Computing. pp. 224-245, 2003.

[69] Zlochiver. S, Rosenfeld. M, Induced-Current Electrical impedance tomography: A 2-D theoretical simulation, IEEE Transactions in Medical Imaging, pp.1550-1661, 2003. 
TRATAMENTO DA CONDIÇÂO DE FRONTEIRA TIPO NEUMANN

\section{Problemas unidimensionais}

Consideramos o seguinte problema:

$$
\left\{\begin{array}{l}
-u^{\prime \prime}(x)=f(x), \quad a<x<b \\
u^{\prime}(a)=\alpha, \quad u^{\prime}(b)=\beta
\end{array}\right.
$$

\section{Condição de compatibilidade:}

Integrando em $(a, b)$ a equação de (A.1):

$$
-\int_{a}^{b} u^{\prime \prime}(x) d x=\int_{a}^{b} f(x) d x
$$

Então:

$$
-\left(u^{\prime}(b)-u^{\prime}(a)\right)=\int_{a}^{b} f(x) d x
$$

assim temos:

$$
\int_{a}^{b} f(x) d x=-(\beta-\alpha)
$$

A equação (A.2) é chamada de condição de compatibilidade. Se esta condição não for satisfeita então não existe solução do problema (A.1).

\section{Soluções de problema (A.1)}

Sejam $v, w$ soluções do problema (A.1) então:

$$
\left\{\begin{array}{l}
-v^{\prime \prime}(x)=f(x)+c d . f r \\
-w^{\prime \prime}(x)=f(x)+c d . f r
\end{array},\right.
$$

onde $c d$. $f r=$ condição de fronteira.

Assim: $-(v-w)^{\prime \prime}(x)=0$, então $-(v-w)^{\prime}(x)=c$, onde $c$ é uma constante. Mas:

$$
\left\{\begin{array}{l}
(v-w)^{\prime}(a)=v^{\prime}(a)-w^{\prime}(a)=\alpha-\alpha=0 \\
(v-w)^{\prime}(b)=v^{\prime}(b)-w^{\prime}(b)=\beta-\beta=0
\end{array},\right.
$$

então: 
$(v-w)^{\prime}(x)=0$, onde temos que:

$$
v(x)-w(x)=c
$$

sendo $C$ é uma constante.

De (A.3) concluímos que o problema (A.1) tem infinitas soluções e que duas soluções diferem em uma constante. Assim, para escolher uma solução é preciso fazer alguma normalização. Por exemplo:

$$
\left\{\begin{array}{l}
\text { Seja: } u(b)-u(a)=\gamma \\
\left(u(b)-\frac{\gamma}{2}\right)-\left(u(a)-\frac{\gamma}{2}\right)=0 \\
v(x)=u(x)-\frac{\gamma}{2}
\end{array}\right.
$$

Note que a solução $v(x)$ tem a propriedade: $v(b)-v(a)=0$.

\section{Solução numérica do problema (A.1)}

Suponha $a=0, b=1$. Fazemos a seguinte subdivisão do intervalo [0,1]:

$$
x_{i}=(i-0.5) h, \quad 1 \leq i \leq M, \text { onde } h=\frac{1}{M}, x_{M}+0.5 h=1.0
$$

A discretização da equação em (A.1) com o método de diferenças finitas centrais fica:

$$
\left\{\begin{array}{l}
-\left(u^{\prime}\right)^{\prime}\left(x_{i}\right)=f\left(x_{i}\right), \quad 1 \leq i \leq M \\
-\left(\frac{u_{i+1 / 2}^{\prime}-u_{i-1 / 2}^{\prime}}{h}\right)=f_{i}=
\end{array}\right.
$$

\section{Sistema linear:}

Para $i=1$

$-\frac{\left(u_{3 / 2}^{\prime}-\alpha\right)}{h}=f_{1}$, então:

$$
\frac{u_{1}-u_{2}}{h^{2}}=f_{1}-\frac{\alpha}{h}
$$


Para $2 \leq i \leq M-1$

$$
\frac{-u_{i-1}+2 u_{i}-u_{i+1}}{h^{2}}=f_{i}
$$

Para $i=M$

$$
\begin{aligned}
& -\left(\frac{\beta-u_{M-1 / 2}^{\prime}}{h}\right)=f_{M} \\
& \frac{u_{M}-u_{M-1}}{h^{2}}=f_{M}+\frac{\beta}{h}
\end{aligned}
$$

Assim o sistema linear fica:

$$
\frac{1}{h^{2}}\left[\begin{array}{cccccccc}
1 & -1 & 0 & & \ldots & & & 0 \\
-1 & 2 & -1 & & & & & \\
0 & -1 & 2 & -1 & & & & \\
\vdots & & & & & & & \\
& & & & -1 & 2 & -1 & \\
0 & & \ldots & & & 0 & -1 & 1
\end{array}\right]\left[\begin{array}{c}
u_{1} \\
u_{2} \\
u_{3} \\
\vdots \\
u_{M-2} \\
u_{M-1} \\
u_{M}
\end{array}\right]=\left[\begin{array}{c}
f_{1}-\frac{\alpha}{h} \\
f_{2} \\
f_{3} \\
\vdots \\
f_{M-2} \\
f_{M-1} \\
f_{M}+\frac{\beta}{h}
\end{array}\right] .
$$

O sistema linear (A.5) é da forma:

$$
A u=b,
$$

onde podemos mostrar que:

- A é simétrica.

- $\quad N(A)=\operatorname{span}\{e\}, e=(1,1, \ldots, 1,1)^{T}$.

Assim $A$ é não inversível. Também temos que:

$$
A u=b \rightarrow e^{T} A u=e^{T} b \rightarrow(A e)^{T} u=e^{T} b \rightarrow e^{T} b=0,
$$

assim: $\operatorname{Im}(A)=\left\{w / e^{T} w=0\right\}=$ Espaço imagem de $A$.

Porém, do sistema linear (A.6): 


$$
e^{T} b=\sum_{i=1}^{M} f_{i}+\left(\frac{\beta-\alpha}{h}\right)=\frac{1}{h}\left(h \sum_{i=1}^{M} f_{i}+(\beta-\alpha)\right)=\frac{1}{h}\left[\int_{a}^{b} f(x) d x+(\beta-\alpha)-\frac{h^{3}}{24} f^{\prime \prime}\left(t_{h}\right)\right]
$$

Mas, pela condição de compatibilidade (A.2):

$$
e^{T} b=-\frac{h^{2}}{24} f^{\prime \prime}\left(t_{h}\right)
$$

onde temos que, geralmente, $e^{T} b \neq 0$. Assim $b \notin \operatorname{Im}(A)$.

Para assegurar (A.7) escolhemos o lado direito da seguinte maneira:

Seja:

$$
\tilde{b}=b-\left(\frac{e^{T} b}{e^{T} e}\right) e
$$

com essa escolha:

$$
e^{T} \tilde{b}=e^{T} b-\left(\frac{e^{T} b}{e^{T} e}\right) e^{T} e=e^{T} b-e^{T} b=0 .
$$

Assim:

* É necessário projetar $b$ na $\operatorname{Im}(A)$ e quando $e=(1,1, \ldots, 1,1)$ temos que:

$$
\tilde{b}_{i}=b_{i}-\frac{1}{M} \sum_{i=1}^{M} b_{i}
$$

Portanto, em vez de resolver o sistema linear (A.6) resolvemos o sistema:

$$
A u=\tilde{b}
$$

O sistema (A.10) tem uma solução mais ela não é única. Duas soluções diferem em uma constante.

Também, seja $\bar{u}$ a solução do sistema linear (A.10) então uma solução numérica “normalizada” é:

$$
\tilde{u}=\bar{u}-\frac{e^{T} u}{e^{T} e} e
$$

onde se $e=(1,1, . ., 1,1)$, temos:

$$
\tilde{u}=\bar{u}-\sum_{i=1}^{M} \bar{u}_{i} / M
$$


Assim temos:

$$
\left\{\begin{array}{l}
v(x)=u(x)+c_{1} \\
\tilde{u}_{i}=\bar{u}_{i}+c_{2}
\end{array},\right.
$$

onde $v(x)$ é a solução exata normalizada e $\tilde{u}$ a solução aproximada. O erro entre esses dois vetores produz:

$$
v_{i}-\tilde{u}_{i}=u_{i}-\bar{u}_{i}+\gamma
$$

Em conseqüência, temos que fazer um refinamento de malha até obter o coeficiente $\gamma$ e comparar $v_{i}$ e $\tilde{u}+\gamma$.

No caso da equação de difusão em coordenadas polares:

$$
\frac{-1}{r}\left[\frac{\partial}{\partial r}\left(\sigma(r, \theta) r \frac{\partial u}{\partial r}\right)+\frac{\partial}{\partial \theta}\left(\frac{\sigma(r, \theta)}{r} \frac{\partial u}{\partial \theta}\right)\right]=f(r, \theta), \quad(r, \theta) \in \Omega
$$

munida da condição de fronteira tipo Neumann:

$$
\left\{\left.\sigma(1, \theta) \frac{\partial u}{\partial r}\right|_{r=1}=g(\theta), \quad \text { em } \partial \Omega\right.
$$

Seja $u(r, \theta)$ uma solução do problema. Então, podemos fazer as seguintes escolhas para temos uma normalização da solução da equação (A.11) e a condição de fronteira (A.12).

1.- Seja:

$$
\begin{aligned}
v(r, \theta) & =u(r, \theta)-\frac{1}{A(\Omega)} \int_{0}^{1} \int_{0}^{2 \pi} u(r, \theta) r d r d \theta= \\
& =u(r, \theta)-\frac{1}{\pi} \int_{0}^{1} \int_{0}^{2 \pi} u(r, \theta) r d r d \theta=u(r, \theta)-\frac{1}{\pi}\left(\sum_{i=1}^{M} \sum_{j=1}^{N} \int_{r_{i-1 / 2}}^{r_{i+1 / 2}} \int_{\theta_{j-1 / 2}}^{\theta_{j+1 / 2}} u(r, \theta) r d r d \theta\right), \\
& =\underset{\substack{\text { Aproximaçá pela fôrmula do } \\
\text { ponto médio }}}{=} u(r, \theta)-\frac{1}{\pi}\left(h_{r} h_{\theta} \sum_{i=1}^{M} \sum_{j=1}^{N} u\left(r_{i}, \theta_{j}\right) r_{i}\right)
\end{aligned}
$$


onde $A(\Omega)$ é a área do domínio $\Omega$ e substituindo os valores de $h_{r}$ e $h_{\theta}$ temos:

$$
v(r, \theta)=u(r, \theta)-\frac{2}{M N} \sum_{i=1}^{M} \sum_{j=1}^{N} u_{i j} r_{i}
$$

Assim, (A.13) constitui uma normalização.

2.- Uma outra escolha é a seguinte:

$$
\begin{aligned}
& v(r, \theta)=u(r, \theta)-\frac{1}{C(\partial \Omega)} \int_{0}^{2 \pi} u(1, \theta) d \theta= \\
& \underset{\substack{\text { Aproximaçã pela fồmula } \\
\text { dos trapezios }}}{=} u(r, \theta)-\frac{1}{L} \sum_{j=1}^{L} u\left(1, j \frac{2 \pi}{L}\right),
\end{aligned}
$$

onde $L$ é suficientemente grande.

Seja o sistema linear:

$$
A \bar{u}=\tilde{b},
$$

onde $A$ é simétrica e $\tilde{b}=b-\frac{e^{T} b}{e^{T} e} e$.

Fazendo uma normalização com a solução aproximada:

$$
\tilde{u}=\bar{u}-\frac{e^{T} u}{e^{T} e} e,
$$

assim:

$$
\tilde{u}=\bar{u}-\frac{1}{M N} \sum_{k=1}^{M N} \bar{u}_{i j} .
$$

Então podemos comparar as soluções exatas normalizadas dadas em (A.13) ou (A.14) com a solução numérica dada em (A.15). 


\section{O JACOBIANO EM TERMOS DA VARIAÇÃO DO POTENCIAL}

Sejam: $I^{d}=\left[I_{1}^{d}, I_{2}^{d}, \ldots, I_{L}^{d}\right]^{T}, d=1,2, . ., P$ os padrões de corrente injetados através dos $L$ eletrodos localizados na fronteira de um domínio condutor $\Omega$ e $\Upsilon^{l}=\left[\Upsilon_{1}^{l}, \Upsilon_{2}^{l}, \ldots, \Upsilon_{L}^{l}\right]^{T}, l=1,2, . ., L$ os padrões de correntes chamados dipolos.

Associamos as distribuições de potencial $u^{d}$ e $\Psi^{l}$ como sendo soluções dos problemas:

$$
\nabla \cdot \sigma \nabla u^{d}=0, \quad \text { em } \Omega
$$

junto à condição de fronteira:

$$
\sigma \frac{\partial u^{d}}{\partial v}=I^{d}=\left\{\begin{array}{l}
\frac{I_{l}}{\left|e_{l}\right|}, x \in e_{l}, l=1,2, . ., L \\
0, x \in \partial \Omega \backslash \bigcup_{l=1}^{L} e_{l}
\end{array},\right.
$$

e

$$
\nabla \cdot \sigma \nabla \Psi^{l}=0, \text { em } \Omega,
$$

onde à condição de fronteira é:

$$
\sigma \frac{\partial \Psi^{l}}{\partial v}=\Upsilon^{l}=\left\{\begin{array}{l}
\frac{-1}{\left|e_{l}\right|}, x \in e_{l}, l=1,2, . ., L \\
\frac{1}{\left|e_{l}\right|}, x \in e_{l+1}, l=1,2, . ., L, \\
0, x \in \partial \Omega \backslash \bigcup_{l=1}^{L} e_{l}
\end{array}\right.
$$

respectivamente.

Sabemos que dados os potenciais elétricos $\phi, \vartheta$ :

$$
\nabla \cdot(\phi \sigma \nabla \vartheta)=\phi \nabla \cdot(\sigma \nabla \vartheta)+\sigma \nabla \phi \cdot \nabla \vartheta
$$

e supondo que os potenciais $\phi$, satisfazem (B.1) ou (B.2), então: 


$$
\iint_{\Omega} \nabla \cdot(\phi \sigma \nabla \vartheta) d V=\iint_{\Omega} \sigma \nabla \phi \cdot \nabla \vartheta d V
$$

usando o teorema da divergência:

$$
\iint_{\Omega} \sigma \nabla \phi \cdot \nabla \vartheta d V=\int_{\partial \Omega} \phi \sigma \frac{\partial \vartheta}{\partial v} d s
$$

onde $v$ é o vetor normal unitário.

De $(* *)$ temos:

$$
\iint_{\Omega} \sigma \nabla u^{d} \cdot \nabla \Psi^{l} d A=\int_{\partial \Omega} u^{d} \sigma \frac{\partial \Psi^{l}}{\partial v} d s=\int_{\partial \Omega} u^{d} \Upsilon^{l} d s=u_{l+1}^{d}-u_{l}^{d}
$$

Definimos operadores não lineares como sendo:

$$
\left[F\left(\sigma, I^{d}\right)\right]_{l}=u_{l+1}^{d}\left(\sigma, I^{d}\right)-u_{l}^{d}\left(\sigma, I^{d}\right)
$$

De $(* *)$ :

$$
\iint_{\Omega} \sigma \nabla \Psi^{l} \cdot \nabla u^{d} d A=\int_{\partial \Omega} \Psi^{l} I^{d} d s
$$

assim, fazendo $\left[F\left(\sigma, I^{d}\right)\right]_{l}=F_{l}^{d}(\sigma)$ :

$$
F_{l}^{d}(\sigma)=\int_{\partial \Omega} \Psi^{l}(\sigma) I^{d} d s
$$

considerando:

$$
\sigma+\delta \sigma \Rightarrow \Psi^{l}(\sigma+\delta \sigma)=\Psi^{l}(\sigma)+\delta \Psi^{l}
$$

então:

$$
F_{l}^{d}(\sigma+\delta \sigma)=F_{l}^{d}(\sigma)+\int_{\partial \Omega} \delta \Psi^{l}(\sigma) I^{d} d s
$$


substituindo (+) em (B.2):

$$
\nabla \cdot\left(\sigma \nabla \Psi^{l}\right)+\nabla \cdot\left(\sigma \nabla\left(\delta \Psi^{l}\right)\right)+\nabla \cdot\left(\delta \sigma \nabla \Psi^{l}\right)+\nabla \cdot\left(\delta \sigma \nabla\left(\delta \Psi^{l}\right)\right)=0
$$

Considerando $\delta \sigma, \delta \Psi^{l}$ mudanças muito pequenas vamos desprezar o último termo da equação anterior e fazemos a seguinte hipótese:

$$
\delta \sigma=0, \text { em } \partial \Omega,
$$

então:

$$
\left\{\begin{array}{l}
\nabla \cdot\left(\sigma\left(\delta \Psi^{l}\right)\right) \simeq-\nabla \cdot\left(\delta \sigma \nabla \Psi^{j}\right) \\
\left.\sigma \frac{\partial\left(\delta \Psi^{l}\right)}{\partial v}\right|_{\partial \Omega}=0
\end{array} .\right.
$$

De $(++)$ :

$$
\int_{\partial \Omega} \delta \Psi^{l}(\sigma) I^{d} d s=\int_{\partial \Omega} \delta \Psi^{l} \sigma \frac{\partial u^{d}}{\partial v} d s=\iint_{\Omega} \sigma \nabla\left(\delta \Psi^{l}\right) \cdot \nabla u^{d} d V,
$$

e seguindo $(*)$ :

$$
\nabla \cdot\left(u^{d} \sigma \nabla\left(\delta \Psi^{l}\right)\right)=\sigma \nabla\left(\delta \Psi^{l}\right) \cdot \nabla u^{d}+u^{d} \nabla \cdot\left(\sigma \nabla\left(\delta \Psi^{l}\right)\right)
$$

então:

$$
\begin{aligned}
& \int_{\partial \Omega}\left(\delta \Psi^{l}\right) I^{d} d s=\iint_{\Omega} \sigma \nabla\left(\delta \Psi^{l}\right) \cdot \nabla u^{d} d V=\iint_{\Omega} \nabla \cdot\left(u^{d} \sigma \nabla\left(\delta \Psi^{l}\right)\right) d V-\iint_{\Omega} u^{d} \nabla \cdot\left(\sigma \nabla\left(\delta \Psi^{l}\right)\right) d V \\
&=\int_{\partial \Omega} u^{d} \sigma \frac{\partial\left(\delta \Psi^{l}\right)}{\partial v} d s-\iint_{\Omega} u^{d} \nabla \cdot\left(\sigma \nabla\left(\delta \Psi^{l}\right)\right) d V \\
&\left(\text { de B.4) }=-\iint_{\Omega} u^{d} \nabla \cdot\left(\sigma \nabla\left(\delta \Psi^{l}\right)\right) d V\right. \\
& \text { (também de B.4) }=\iint_{\Omega} u^{d} \nabla \cdot\left(\delta \sigma \nabla \Psi^{l}\right) d A .
\end{aligned}
$$

De (*):

$$
\nabla \cdot\left(u^{d} \delta \sigma \nabla \Psi^{l}\right)=u^{d} \nabla \cdot\left(\delta \sigma \nabla \Psi^{l}\right)+\delta \sigma \nabla \Psi^{l} \cdot \nabla u^{d}
$$


então:

$$
\begin{aligned}
\int_{\partial \Omega}\left(\delta \Psi^{l}\right) I^{d} d s=\iint_{\Omega} u^{d} \nabla \cdot\left(\delta \sigma \nabla \Psi^{l}\right) d V & =\iint_{\Omega} \nabla \cdot\left(u^{d} \delta \sigma \nabla \Psi^{l}\right) d V-\iint_{\Omega} \delta \sigma \nabla \Psi^{l} \cdot \nabla u^{d} d V \\
& =\int_{\partial \Omega} u^{d} \delta \sigma \frac{\partial\left(\Psi^{l}\right)}{\partial v} d s-\iint_{\Omega} \delta \sigma \nabla \Psi^{l} \cdot \nabla u^{d} d V \\
\text { (de B.3) } & =-\iint_{\Omega} \delta \sigma \nabla \Psi^{l} \cdot \nabla u^{d} d V
\end{aligned} .
$$

Conseqüentemente:

$$
\int_{\partial \Omega}\left(\delta \Psi^{l}\right) I^{d} d s=-\iint_{\Omega}(\delta \sigma) \nabla \Psi^{l} \cdot \nabla u^{d} d V,
$$

e usando $(++)$ :

$$
F_{l}^{d}(\sigma+\delta \sigma) \simeq F_{l}^{d}-\iint_{\Omega}(\delta \sigma) \nabla \Psi^{l} \cdot \nabla u^{d} d V
$$

Finalmente, discretizando o domínio $\Omega$ em ne volumes finitos, temos:

$$
F_{l}^{d}(\sigma+\delta \sigma) \simeq F_{l}^{d}(\sigma)-\sum_{k=1}^{n e}\left(\delta \sigma_{k}\right) \iint_{\Omega_{k}} \nabla \Psi^{l} \cdot \nabla u^{d} d A
$$

Portanto:

$$
\frac{\partial F_{l}^{d}}{\partial \sigma_{k}}=-\iint_{\Omega_{k}} \nabla \Psi^{l} \cdot \nabla u^{d} d A
$$

onde $\quad d=1,2,3, \ldots, P$ padrões de corrente, $I=1,2,3, \ldots, L$ eletrodos e $k=1,2,3, \ldots$, ne volumes finitos. 